Aus der Klinik für Allgemein-, Viszeral- und Kinderchirurgie

(Prof. Dr. med. M. Ghadimi)

der Medizinischen Fakultät der Universität Göttingen

\title{
Diagnostische Varianzen bei \\ Ösophagus- und Magentumoren
}

\author{
INAUGURAL-DISSERTATION \\ zur Erlangung des Doktorgrades \\ der Medizinischen Fakultät der \\ Georg-August-Universität Göttingen
}

vorgelegt von

Maria Diekhoff

aus

Siegburg

Göttingen 2018 
Dekan:

Referent/in

Ko-Referent/in:

Drittreferent/in:
Prof. Dr. rer. nat. H.K. Kroemer

Prof. Dr. med. J. Gaedcke

PD Dr. F. Bremmer

Prof. Dr. M. Schön

Datum der mündlichen Prüfung: 10.04.2019 
Hiermit erkläre ich, die Dissertation mit dem Titel "Diagnostische Varianzen bei Ösophagus- und Magentumoren" eigenständig angefertigt und keine anderen als die von mir angegebenen Quellen und Hilfsmittel verwendet zu haben.

Göttingen, den 29. Juli 2018 


\section{Inhaltsverzeichnis}

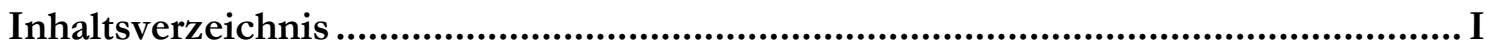

Abbildungsverzeichnis ....................................................................................... III

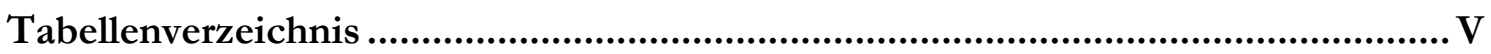

Abkürzungsverzeichnis.........................................................................................

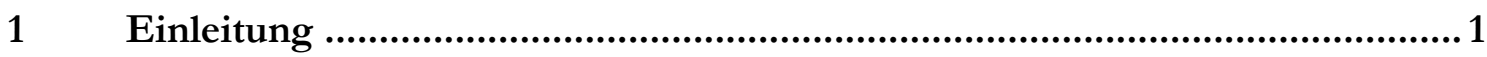

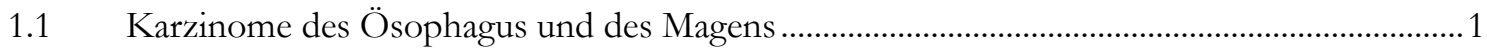

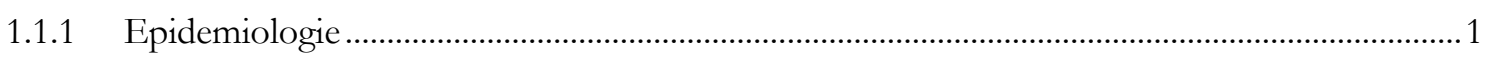

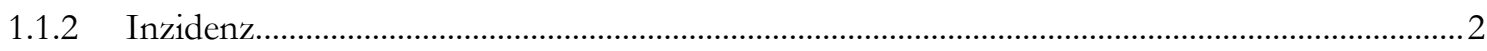

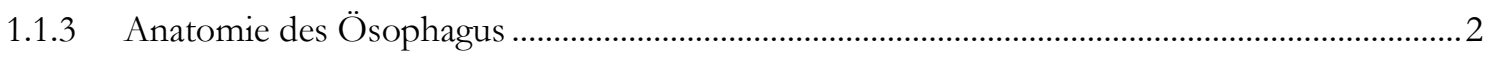

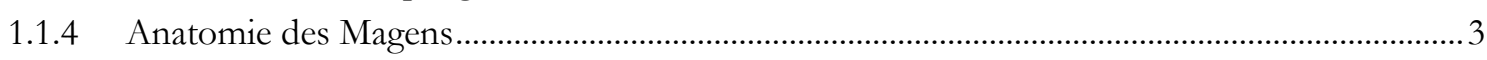

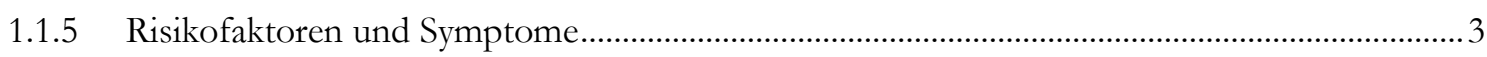

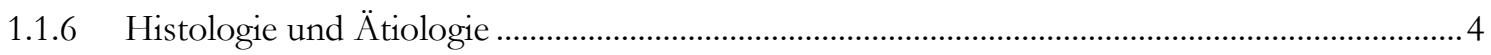

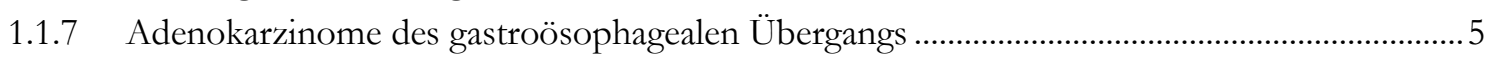

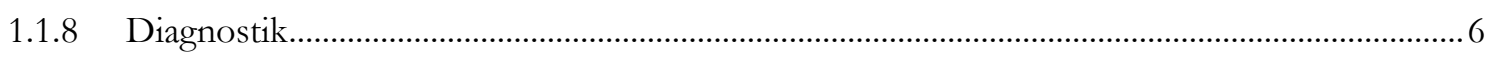

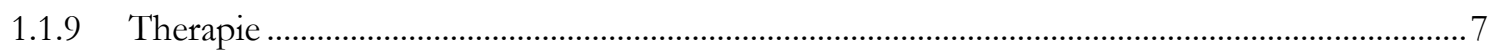

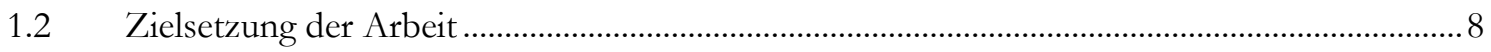

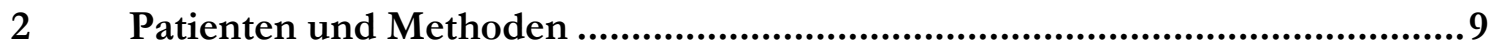

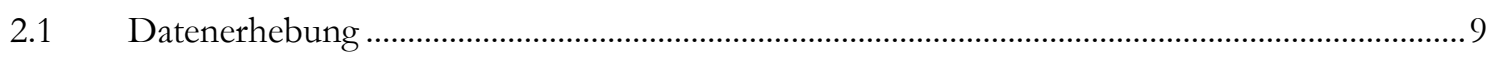

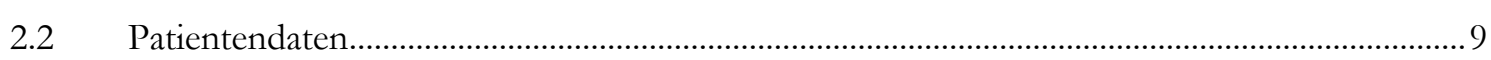

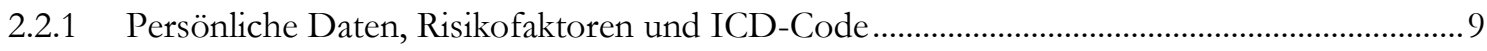

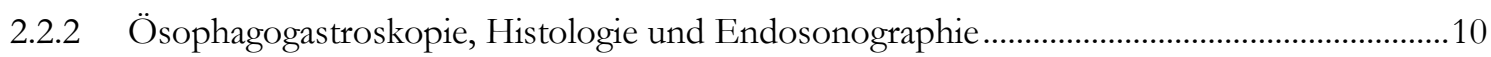

2.2.3 Neoadjuvante Chemo- und Strahlentherapie.....................................................................12

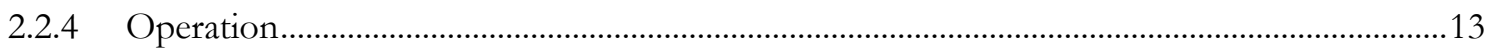

2.2.5 Postoperative Histologie und Resektionsstatus.......................................................................13

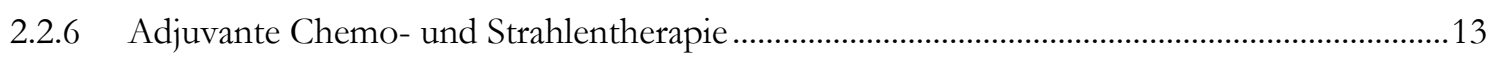

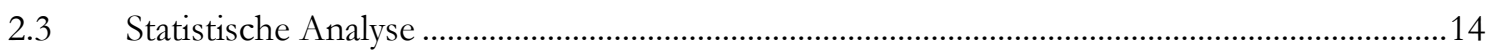

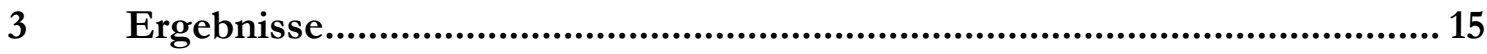

3.1 Häufigkeitsverteilung des Patientenkollektivs.........................................................................15

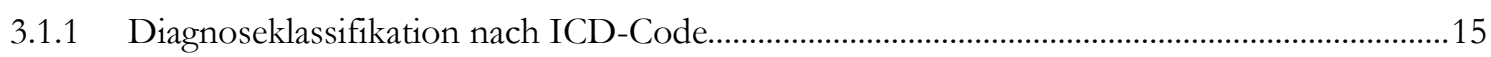

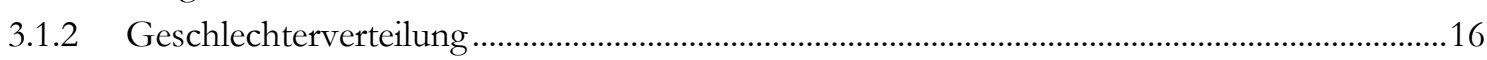

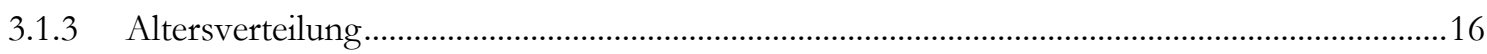

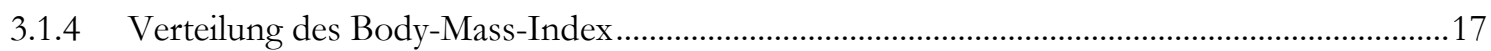

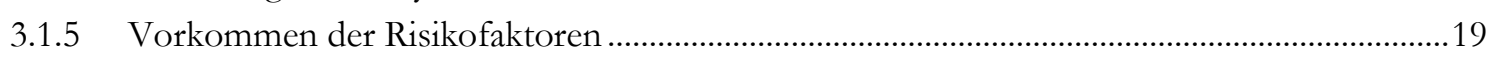

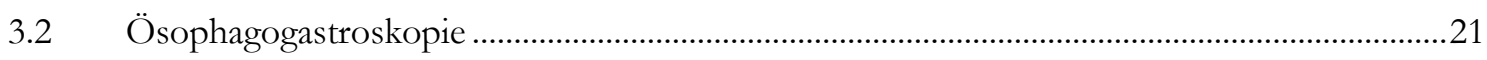

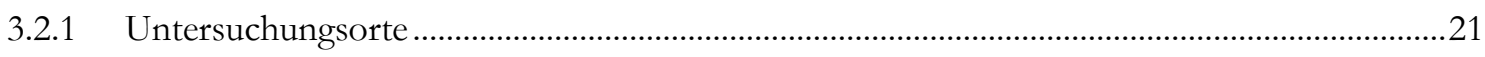

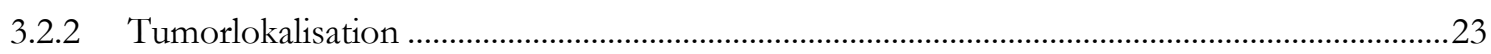




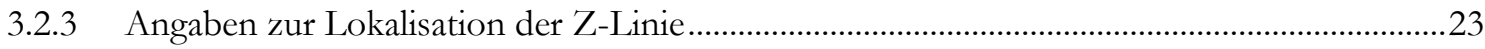

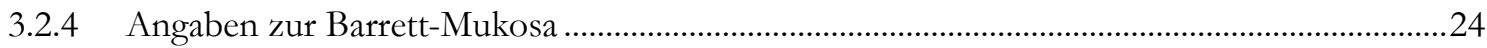

3.2.5 Histologische Auswertung der in der Ösophagogastroskopie gewonnenen Biopsie .............27

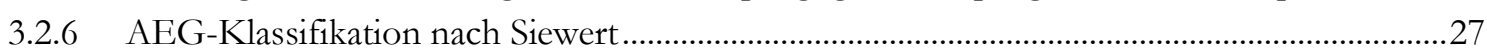

3.2.7 Vergleich der Angaben von den an der Universitätsmedizin Göttingen durchgeführten

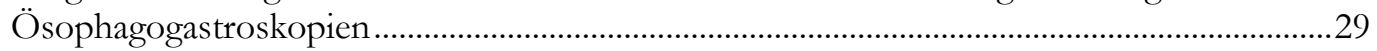

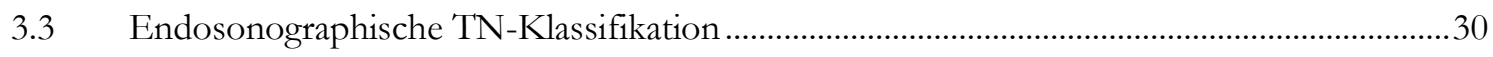

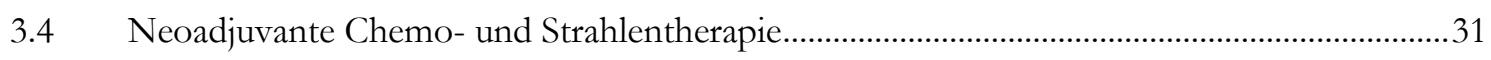

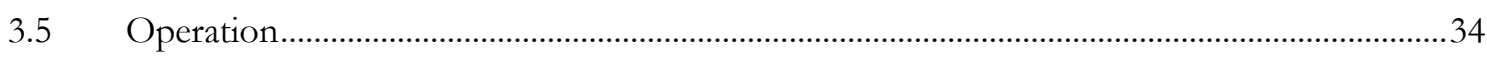

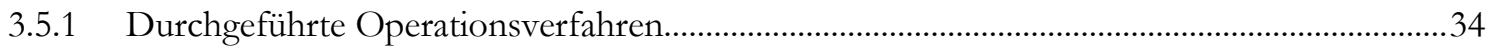

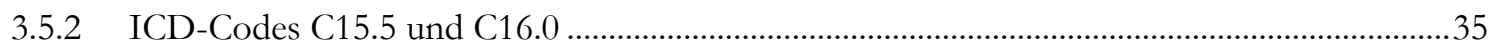

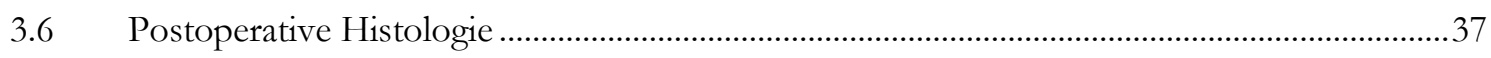

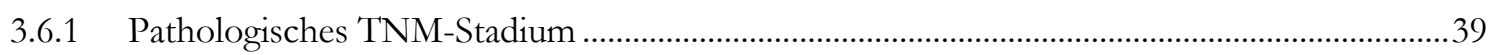

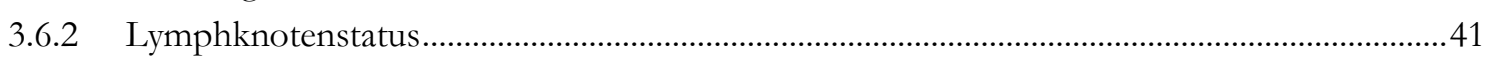

3.6.3 Barrett-Karzinom - Adenokarzinom auf dem Boden eines Barrett-Ösophagus ...................43

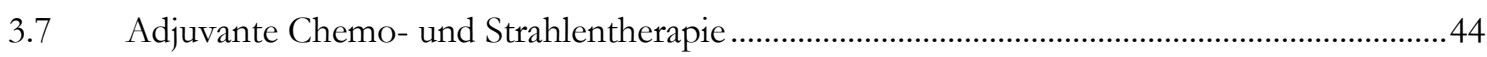

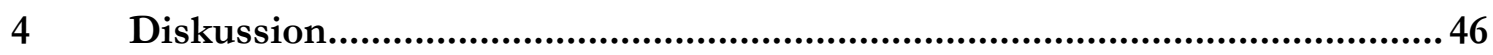

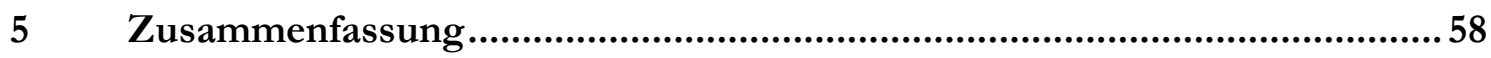

$6 \quad$ Literaturverzeichnis ....................................................................... 61 


\section{Abbildungsverzeichnis}

Abbildung 1: Geschlechterverteilung des Gesamtkollektivs sowie der beiden Untergruppen (Patienten mit bösartiger Neubildung des Ösophagus bzw. des Magens).

Abbildung 2: Altersverteilung des Gesamtkollektivs und der beiden Untergruppen (Patienten mit der Diagnose Ösophagus- bzw. Magenkarzinom).

Abbildung 3: Verteilung des Body-Mass-Index bei Frauen im Gesamtkollektiv und in den beiden Untergruppen (Patienten mit der Diagnose Ösophagus- bzw. Magenkarzinom) sowie der Verteilung in der Bundesrepublik Deutschland..

Abbildung 4: Verteilung des Body-Mass-Index bei Männern im Gesamtkollektiv und in den beiden Untergruppen (Patienten mit der Diagnose Ösophagus- bzw. Magenkarzinom) sowie der Verteilung in der Bundesrepublik Deutschland...

Abbildung 5: Verteilung des Body-Mass-Index in den jeweiligen Gruppen der Patienten mit einem Plattenepithel- bzw. Adenokarzinom.

Abbildung 6: Vorkommen von einigen Risikofaktoren im Kollektiv der Patienten mit einem Ösophaguskarzinom $(\mathrm{n}=103)$.

Abbildung 7: Vorkommen von einigen Risikofaktoren im Kollektiv der Patienten mit einem Magenkarzinom $(\mathrm{n}=143)$.

Abbildung 8: Graphische Darstellung der unterschiedlichen Untersuchungsorte für die erste Ösophagogastroskopie ( $\mathrm{n}=234)$.

Abbildung 9: Graphische Darstellung der unterschiedlichen Untersuchungsorte für die zweite Ösophagogastroskopie $(\mathrm{n}=136)$.

Abbildung 10: Verteilung der vorliegenden Tumorlokalisationen entsprechend den Befunden von Endoskopie I ( $\mathrm{n}=234)$ und Endoskopie II ( $\mathrm{n}=136)$.

Abbildung 11: Dokumentation der Z-Linie als Zentimeterangabe nach der ersten Ösophagogastroskopie an der Universitätsmedizin Göttingen sowie außerhalb.

Abbildung 12: Dokumentation der Z-Linie als Zentimeterangabe nach der zweiten Ösophagogastroskopie an der Universitätsmedizin Göttingen sowie außerhalb.

Abbildung 13: Verteilung der vorgenommenen Angaben bezüglich eines möglichen Vorliegens einer Barrett-Mukosa für Endoskopie I ( $\mathrm{n}=234)$ und Endoskopie II $(\mathrm{n}=136)$..

Abbildung 14: Verteilung der in Endoskopie I vorliegenden Barrett-Mukosa-Befunde für die Patienten entsprechend der für sie zutreffenden ICD-Klassifikation $(n=34)$.......................26

Abbildung 15: Verteilung der in Endoskopie II vorliegenden Barrett-Mukosa-Befunde für die Patienten entsprechend der für sie zutreffenden ICD-Klassifikation $(\mathrm{n}=15)$. .......................26

Abbildung 16: Verteilung der präoperativen Differenzierungsgrade im Gesamtkollektiv $(\mathrm{n}=243)$.

Abbildung 17: Verteilung der vorgenommenen Angaben bezüglich einer möglichen Einteilung entsprechend der Siewert-Klassifikation nach erster und zweiter Ösophagogastroskopie.

Abbildung 18: Verteilung der angegebenen Siewert-Klassifikationen (AEG-Typ-I, -II und -III) nach Ösophagogastroskopie I und II.

Abbildung 19: Verteilung der Stadiengruppierungen nach UICC-Kriterien bei Patienten mit einem Ösophaguskarzinom, die neoadjuvant keine Therapie erhalten haben $(\mathrm{n}=35)$..........32

Abbildung 20: Verteilung der Stadiengruppierungen nach UICC-Kriterien bei Patienten mit einem Ösophaguskarzinom, die neoadjuvant eine Chemotherapie erhalten haben $(\mathrm{n}=12)$.

Abbildung 21: Verteilung der Stadiengruppierungen nach UICC-Kriterien bei Patienten mit einem Ösophaguskarzinom, die neoadjuvant eine Radiochemotherapie erhalten haben $(\mathrm{n}=56)$. 
Abbildung 22: Verteilung der UICC-Stadiengruppierungen bei Patienten mit einem Magenkarzinom, die neoadjuvant keine Therapie erhalten haben $(n=66)$. .

Abbildung 23: Verteilung der UICC-Stadiengruppierungen bei Patienten mit einem Magenkarzinom, die neoadjuvant eine Chemotherapie erhalten haben $(n=73)$

Abbildung 24: Verteilung der UICC-Stadiengruppierungen bei Patienten mit einem Magenkarzinom, die neoadjuvant eine Radiochemotherapie erhalten haben $(n=4)$............34

Abbildung 25: Verteilung der in den Endoskopiebefunden dokumentierten Angaben bezüglich einer Einteilung anhand der Siewert-Klassifikation bei Patienten mit der Diagnose ICD-C15.5 oder ICD-C16.0 $(n=118)$.

Abbildung 26: Graphische Darstellung der Verteilung des pathologischen T-Stadiums der beiden Patientengruppen.

Abbildung 27: Graphische Darstellung der Verteilung des pathologischen N-Stadiums der beiden Patientengruppen.

Abbildung 28: Graphische Darstellung der Verteilung des pathologischen M-Stadiums der beiden Patientengruppen.

Abbildung 29: Grafik zur Verteilung der Anzahl der untersuchten Lymphknoten bei den Patienten, die aufgrund eines Ösophaguskarzinoms $(n=99)$ oder eines Magenkarzinoms $(\mathrm{n}=141)$ operiert wurden.

Abbildung 30: Grafik zur Verteilung der Anzahl der befallenen Lymphknoten bei den Patienten, die aufgrund eines Ösophaguskarzinoms $(n=99)$ oder eines Magenkarzinoms $(\mathrm{n}=141)$ operiert wurden.

Abbildung 31: Grafik zur Verteilung des Verhältnisses von befallenen zu untersuchten Lymphknoten bei den Patienten, die aufgrund eines Ösophaguskarzinoms ( $=99)$ oder eines Magenkarzinoms ( $\mathrm{n}=141)$ operiert wurden...

Abbildung 32: Graphische Darstellung zu den prä- und postoperativen Aussagen zum BarrettKarzinom.

Abbildung 33: Darstellung des Einsatzes adjuvanter Therapieoptionen bei Patienten mit einem Magenkarzinom $(\mathrm{n}=143)$.

Abbildung 34: Darstellung des Einsatzes adjuvanter Therapieoptionen bei Patienten mit einem Ösophaguskarzinom $(\mathrm{n}=103)$ 


\section{Tabellenverzeichnis}

Tabelle 1: Aufteilung des Gesamtkollektivs in Bezug auf die bestehende Diagnose anhand des ICD-Codes.

Tabelle 2: Gegenüberstellung der Häufigkeit der dokumentierten Angaben bei den an der

UMG durchgeführten Ösophagogastroskopien. Endoskopie I ( $n=35)$, II ( $n=73)$ .30

Tabelle 3: Verteilung des klinischen T-und N-Stadiums nach Endosonographie I und II.

Tabelle 4: Gegenüberstellung der Einteilung entsprechend der TNM-Klassifikation von den

Patienten, bei denen Endosonographie I und II durchgeführt wurden ( $n=13)$.

Tabelle 5: Verteilung der beim Gesamtkollektiv durchgeführten Operationsverfahren $(n=240)$......35

Tabelle 6: Verteilung der neoadjuvant und/oder adjuvant durchgeführten Therapien bei Patienten mit der Diagnose ICD-C15.5 oder ICD-C16.0. .36

Tabelle 7: Darstellung der durchgeführten Operationsverfahren bei Patienten mit der Diagnose ICD-C15.5 oder ICD-C16.0.

Tabelle 8: Verteilung der Histologiebefunde der an der Universitätsmedizin Göttingen operierten Patienten $(\mathrm{n}=240)$

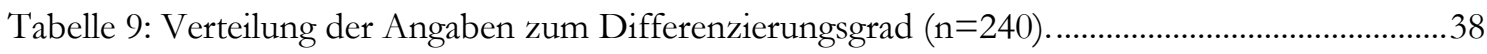

Tabelle 10: Verteilung der Angaben zum Resektionsstatus ( $\mathrm{n}=240)$

Tabelle 11: Verteilung der pathologischen TNM-Stadien für die Gruppe der neoadjuvant behandelten Patienten sowie für die Patientengruppe, bei der keine neoadjuvante Therapie stattgefunden hat. 


\section{Abkürzungsverzeichnis}

$\begin{array}{ll}\text { AEG-Tumor } & \text { Adenokarzinom des gaströ̈sophagealen Übergangs } \\ \text { BMI } & \text { Body-Mass-Index } \\ \text { G-CCC } & \text { Göttinger Comprehensive Cancer Center } \\ \text { GEKID } & \text { Gesellschaft der epidemiologischen Krebsregister in Deutschland e.V. } \\ \text { ICD } & \text { International statistical classification of diseases and related health } \\ & \text { problems } \\ \text { LAE } & \text { Lymphadenektomie } \\ \text { RKI } & \text { Robert Koch-Institut } \\ \text { TNM } & \text { Tumor-Nodus-Metastasen } \\ \text { UICC } & \text { Union internationale contre le cancer } \\ \text { UMG } & \text { Universitätsmedizin Göttingen } \\ \text { WHO } & \text { World Health Organization }\end{array}$




\section{$1 \quad$ Einleitung}

\subsection{Karzinome des Ösophagus und des Magens}

\subsubsection{Epidemiologie}

Im Jahr 2012 sind in Deutschland insgesamt 125,1 Männer und 83,7 Frauen je 100.000 Einwohner aufgrund einer Krebserkrankung verstorben. Das Ösophaguskarzinom befand sich bei Männern mit 4,8 jährlichen Todesfällen pro 100.000 Einwohner auf dem neunten Platz $(2,8 \%)$ der häufigsten Krebstodesursachen in Deutschland. Bei Frauen belegte es mit jährlich 1,1 Todesfällen pro 100.000 Einwohner (1,3\%) den neunzehnten Platz (dkfz 2012). Das Magenkarzinom stand bei Männern mit jährlich 5,9 Todesfällen pro 100.000 Einwohner an fünfter Stelle (4,7\%). Es folgte damit auf Lungenkrebs, Dick- und Enddarmtumoren, Prostata- sowie Bauchspeicheldrüsenkrebs, welche die vier häufigsten Krebstodesursachen bei Männern in Deutschland waren. Bei Frauen stand das Magenkarzinom mit 3,1 Todesfällen pro 100.000 Einwohner pro Jahr (3,8\%), nach Brustkrebserkrankungen, Lungen-, Dick- und Enddarmtumoren, Bauchspeicheldrüsenund Eierstockkrebs an sechster Stelle (dkfz 2012). Die gemeinsame Veröffentlichung des Robert Koch-Instituts und der Gesellschaft der epidemiologischen Krebsregister in Deutschland (2015) zeigte folgende Neuerkrankungsraten für das Magen sowie das Ösophaguskarzinom: Im Jahr 2012 waren insgesamt 5.030 Männer und 1.510 Frauen an einem neuaufgetretenen Ösophaguskarzinom erkrankt. Karzinome des Ösophagus traten demnach in Deutschland bei Männern deutlich häufiger auf als bei Frauen. Jedoch wurde bei beiden Geschlechtern ein Anstieg der Erkrankungsrate prognostiziert, der bei Männern hingegen deutlicher ausfiel als bei Frauen (rohe Erkrankungsrate je 100.000 Personen: Männer: von 12,8\% auf 13,9\%; Frauen: von 3,7\% auf 3,9\%). Das mittlere Erkrankungsalter bei Männern lag 2012 bei 67 Jahren. Frauen erkrankten im Schnitt vier Jahre später mit 71 Jahren. Aufgrund großer Fortschritte, die in der Diagnostik und Therapie des Ösophaguskarzinoms erreicht worden sind, besserten sich die 5-Jahres-Überlebensraten für Patienten mit Speiseröhrenkrebs seit den 1980er-Jahren deutlich. Die Prognose bleibt jedoch nach wie vor ungünstig (RKI 2010a). Die relativen 5-Jahres-Überlebensraten beliefen sich bei Männern auf 22\% und bei Frauen auf 24\%. Beim Magenkarzinom gab es im Jahr 20129.180 Neuerkrankungen bei Männern und 6.460 Neuerkrankungen bei Frauen. Die Prognose des Robert Koch-Instituts sagte einen leichten Rückgang der Neuerkrankungsrate für beide Geschlechter voraus (rohe Erkrankungsrate je 100.000 Personen: Männer: von 23,4\% auf 23,1\%; Frauen: von 15,7\% auf 15,4\%). Für die Zahl der Erkrankungen an Magenkrebs zeigte sich in Deutschland in den letzten Jahrzehnten ein konstanter Rückgang (RKI 2010a). Männer erkrankten 2012 an einem Magenkarzinom im 
Mittel mit 72 Jahren durchschnittlich drei Jahre früher als Frauen mit einem mittleren Erkrankungsalter von 75 Jahren. Mit 32\% bei Männern und 33\% bei Frauen sind die relativen 5-Jahres-Überlebensraten beim Magenkarzinom etwas besser als die für das Ösophaguskarzinom angegebenen Werte.

\subsubsection{Inzidenz}

Das Vorkommen von Adenokarzinomen ist in den letzten Jahren auf etwa ein Drittel aller Tumoren der Speiseröhre angestiegen (RKI 2015), unter anderem aufgrund von einer Zunahme der Zahl der Neuerkrankungen an einem Barrett-Ösophagus in den westlichen Ländern. Vor allem bei männlichen Patienten unter 60 Jahren kam es zu einer deutlichen Steigerung der Inzidenz (van Soest 2005). Bei Ösophagus- und Magentumoren ist der Anteil des Adenokarzinoms im Gegensatz zum Plattenepithelkarzinom stark angestiegen. In den USA kam es einer Studie zufolge bei weißen Männern zu einer deutlichen Inzidenzsteigerung in den Jahren von 1974 bis 1994. Mit einem Anstieg der Inzidenz von $350 \%$ übertraf dort das Adenokarzinom des Ösophagus das Plattenepithelkarzinom. In einem geringeren Maße von 150\% kam es auch zu einem Anstieg kardialer Adenokarzinome, die somit in etwa der Rate von nicht kardial gelegenen Magenkarzinomen entsprechen (Devesa et al. 1998).

\subsubsection{Anatomie des Ösophagus}

Der Ösophagus schließt sich an den Pharynx an und verbindet diesen mit dem Magen. Es handelt sich um einen muskulären Schlauch, welcher sich vor der Wirbelsäule befindet und in drei Teile eingeteilt wird (Pars cervicalis, thoracica und abdominalis). Der Ösophagus ist ungefähr 23-26 cm lang und beginnt am unteren Rand des Ringknorpels. Dieser Ösophagusmund ist etwa 14-15 cm von der vorderen Zahnreihe entfernt. Vor dem 11.-12. Brustwirbel geht die Speiseröhre an der Kardia in den Magen über. Die Entfernung von der vorderen Zahnreihe bis in den Magen beträgt 37-41 cm, wobei die individuellen Angaben von der Rumpfgröße des jeweiligen Menschen abhängig sind. Im Verlauf der Speiseröhre werden drei anatomische Engen beschrieben. Bei der ersten Enge handelt es sich um den Ösophagusmund, welche etwa $15 \mathrm{~cm}$ aboral der Zahnreihe liegt und die engste Stelle darstellt. Die zweite Engstelle ist die sogenannte Aortenenge. Diese befindet sich $25 \mathrm{~cm}$ aboral der vorderen Zahnreihe und stellt den Bereich dar, an dem Aortenbogen und linker Bronchus in enger Nachbarschaft zum Ösophagus liegen. Die dritte Enge, die durch das Zwerchfell entsteht, befindet sich oral der Kardia, etwa $40 \mathrm{~cm}$ von der Zahnreihe entfernt. Die Zwerchfellenge wird durch die Kontraktion der in diesem Gebiet kräftigen Ringmuskulatur erzeugt, wodurch es zur Ausbildung einer Hiatushernie kommen kann. In einem solchen Fall liegt keine Enge vor, es ist allerdings auch kein Schluss möglich, wodurch es vermehrt zu Reflux kommt. Des Weiteren wird durch das Vorliegen einer Hiatushernie die Orientierung während einer Endoskopie erschwert. 
Der Wandaufbau des Ösophagus teilt sich in vier Schichten. Von innen nach außen sind dies:

- Tunica mucosa (mehrschichtiges, unverhorntes Plattenepithel und Lamina propria)

- Tunica submucosa (enthält Lymph- und Blutgefäße, vegetativen Plexus, Schleimdrüsen)

- Tunica muscularis (Muskelbündel in wechselnden steilen oder schraubenförmigen Touren)

- Tunica adventitia (äußere Umhüllung aus lockerem Bindegewebe mit Gefäßen, Nerven und glatten Muskelfasern)

Funktionell ist die Speiseröhre für den Weitertransport von Nahrungsbestandteilen und Flüssigkeiten vom Pharynx in den Magen zuständig. Im Anschluss an den Schluckakt wird im Pharynx eine peristaltische Welle ausgelöst. Es kommt zu Kontraktionen von 2-4 cm langen Ösophagusarealen, durch die Speisen und Flüssigkeiten in den Magen befördert werden. Durch Druck von Nahrungsbestandteilen auf die Ösophaguswand entstehen sekundäre peristaltische Wellen und es kommt $\mathrm{zu}$ einer Öffnung des unteren Ösophagussphinkters für 5-8 Sekunden. Es handelt sich beim unteren Ösophagussphinkter nicht um einen eigentlichen Sphinktermuskel. Vielmehr führen mehrere Faktoren zu einem suffizienten Verschluss des Ösophagus wie zum Beispiel ein im unteren Abschnitt der Speiseröhre bestehender höherer muskulärer Druck. Außerdem steht der Ösophagus unter einer starken Längsspannung, welche ihn in angespanntem Zustand verschlossen hält. Des Weiteren ist die leicht gewinkelte Einmündung in den Magen eine Ursache für den ausreichenden Verschluss (Elmar et al. 2003).

\subsubsection{Anatomie des Magens}

Der Magen schließt sich an die Speiseröhre an und gliedert sich in mehrere Teile (Kardia, Fundus, Korpus, Pylorus). Die Größe des Magens passt sich den individuellen Essgewohnheiten an. Durchschnittlich hat der Magen eines Erwachsenen ein Fassungsvermögen von 1,5 l. Ist der Magen ungefüllt, dann liegen die stark kontrahierten und mit Magenschleim bedeckten Wände des Korpus aneinander. Der Magenfundus ist jedoch durch die geschluckte Luft gedehnt. Die Wandschichtung besteht wie im übrigen Darmrohr aus Tunica mucosa, Tunica muscularis und Tunica serosa (bei intraperitonealer Lage, sonst Adventitia). Die Tunica mucosa weist eine Lamina muscularis mucosae, eine Lamina propria und ein hochprismatisches, einschichtiges Epithel auf. In der Pars cardiaca geht die grau-rötliche Schleimhaut des Magens in der Ora serrata, einer gezackten Linie, nach oral in die Schleimhaut der Speiseröhre über, welche sich blasser darstellt. Der Magen hat neben mechanischen auch chemische Funktionen (Elmar et al. 2003).

\subsubsection{Risikofaktoren und Symptome}

Als Risikofaktoren für die Entstehung eines Plattenepithelkarzinoms der Speiseröhre führt die 2015 veröffentlichte S3-Leitlinie Ösophaguskarzinom Alkohol, Strahlentherapie im 
Hals-Thorax-Bereich sowie synchrone und metachrone Kopf- und Halstumoren auf. Gemeinsame Risikofaktoren für Plattenepithel- und Adenokarzinome sind demnach Rauchen, das Vorhandensein einer Achalasie und Stenosen nach Säure- und Laugenverätzungen. Übergewicht und Adipositas, gastroösophagealer Reflux und das Vorliegen eines Barrett-Ösophagus werden als Risikofaktoren für die Entstehung eines Adenokarzinoms aufgeführt. Hierbei steht das Vorhandensein eines Barrett-Ösophagus besonders im Vordergrund, da durch die Zylinderepithelmetaplasie das Risiko etwa 30fach erhöht ist (Fritz 2006). Für das Magenkarzinom werden in der entsprechenden Leitlinie die folgenden Risikofaktoren genannt: bakterielle Infektionen des Magens mit Helicobacter pylori, das höhere Alter des Patienten, das Vorliegen einer perniziösen Anämie, ein sozioökonomisch niedriger Status sowie das Leben in einer Hochrisikopopulation, Tabakund Alkoholkonsum, vorherige Magenoperationen, familiäre Vorbelastung des Patienten sowie Umwelt- und Ernährungsfaktoren. Frühsymptome sind bei Ösophagus- und Magenkarzinomen selten. Die Dysphagie wird als ein typisches Zeichen beurteilt, welches sich schleichend einstellt und als Spätsymptom zu werten ist (Menges 2004). Zur Dysphagie zählen neben Schluckstörungen auch Schmerzen oder ein Organfühlen beim Schlucken. In den meisten Fällen kommt es erst zu den beschriebenen Symptomen, wenn bereits zwei Drittel des Speiseröhrenlumens durch den Tumor verlegt wurden (Frieling 2000). Die Leitlinie zum Magenkarzinom von 2012 nennt neben der Dysphagie noch weitere Alarmsymptome, die im klinischen Zusammenhang mit dem Verdacht auf ein Karzinom des Ösophagus oder Magens zu einer Endoskopie mit der Entnahme von Biopsien führen sollten. Diese Alarmsymptome sind rezidivierendes Erbrechen, Inappetenz, Gewichtsverlust und gastrointestinale Blutungen.

\subsubsection{Histologie und Ätiologie}

Bei Speiseröhrentumoren sind die beiden häufigsten histopathologischen Formen das Plattenepithel- und das Adenokarzinom (Halm und Witzigmann 2005). Bei den Karzinomen des Magens handelt es sich meist um Adenokarzinome (Riede und Blum 2009). Seltenere Tumoren des Magens sind vom Mesenchym ausgehende gastrointestinale Stromatumoren (GIST) (Stamatakos et al. 2009) oder MALT-Lymphome (Mucosa Associated Lymphoid Tissue) (Fischbach 1997). Ziel dieser Arbeit war die Auswertung epithelialer Tumoren, weshalb im Weiteren auch nur auf diese eingegangen wird.

Die Schleimhaut des Ösophagus ist mit Plattenepithel ausgekleidet. Die Mehrzahl aller Karzinome der Speiseröhre entsteht auf dem Boden von Metaplasien dieser Zellen, und es handelt sich somit um Plattenepithelkarzinome. Diese Karzinome können mithilfe einer Klassifikation der World Health Organization (WHO) weiter als warziges oder basales Plattenzellkarzinom sowie als Spindelzellkarzinom beschrieben werden. Außerdem ist eine Einstufung in gut, mäßig oder schlecht differenzierte Karzinome möglich (Watanabe et al. 1990). Bei einem Plattenepithelkarzinom des Ösophagus liegt der vorwiegende Entstehungsort im mittleren bis unteren Drittel der Speiseröhre (Nigg et al. 2006). Durch 
chronischen Reflux von Magensäure in die Speiseröhre kann es zu einer Umwandlung des Plattenepithels in schleimbildendes prismatisches Epithel kommen (s.u. 1.1.3 Risikofaktoren). Kommt es im unteren Drittel des Ösophagus oberhalb des gastroösophagealen Übergangs zu dem beschriebenen Ersatz des Plattenepithels durch Zylinderepithel, so bezeichnet man diese intestinale Metaplasie als Barrett-Mukosa. Sie wird in eine kurzstreckige $(<3 \mathrm{~cm})$ und eine langstreckige $(>3 \mathrm{~cm})$ Barrett-Mukosa unterteilt. Es handelt sich hierbei um eine fakultative Präkanzerose. Von dieser geht ein erhöhtes Entartungsrisiko für die Entstehung eines Adenokarzinoms aus (Welsch 2009). In den meisten Fällen kommt es $\mathrm{zu}$ einer Metaplasie aufgrund einer bestehenden Refluxösophagitis. Von den Patienten mit Symptomen eines gastroösophagealen Reflux findet sich bei 5-10\% eine solche Umwandlung des Epithels. Es kommt zu einem Ersatz des weißlichen Plattenepithels der Speiseröhre durch rötliche Schleimhaut mit zungenförmigen Ausläufern Richtung oral (Jochum und Barreton 2012).

Die Klassifikation der WHO ermöglicht ebenfalls eine Einstufung der epithelialen Tumoren des Magens. Die histologische Einstufung des Magenkarzinoms ist unter anderem möglich als ein Adenokarzinom, ein papilläres, tubuläres oder ein muzinöses Adenokarzinom, ein Siegelringzell- oder Plattenzellkarzinom sowie ein kleinzelliges oder ein undifferenziertes Karzinom (Watanabe et al. 1990). Außerdem ist eine Zuordnung anhand der Laurén-Klassifikation möglich. Hier gibt es das Karzinom vom intestinalen Typ und das Karzinom vom diffusen Typ (Von Herbay et al. 2004). Besteht ein Karzinom aus Anteilen vom intestinalen sowie vom diffusen Typ, wird es als Karzinom vom Mischtyp klassifiziert (Jochum 2008).

\subsubsection{Adenokarzinome des gastroösophagealen Übergangs}

Adenokarzinome des gastroösophagealen Übergangs (AEG-Tumoren) gehören weltweit zu einer der am stärksten zunehmenden malignen Erkrankungen (Schoppmann 2013). Im Jahre 1987 wurde von Siewert et al. eine Klassifikation zur topographischen Einordnung dieser Übergangstumoren veröffentlicht. Im weiteren Verlauf der Arbeit wird diese Einteilung wie im klinischen Alltag üblich als Siewert-Klassifikation bezeichnet.

Um eine exakte Zuordnung des Befundes anhand der Siewert-Klassifikation zu ermöglichen, ist eine Erläuterung des Begriffs Kardia notwendig. Anatomisch stellt das untere Ende des unteren Ösophagussphinkters die Kardia dar. Dieser Sphinkter liegt demzufolge am Übergang der zweischichtigen ösophagealen Tunica muscularis zur dreischichtigen Tunica muscularis des Magens. Anhand dieses Kriteriums ist es allerdings nicht möglich, während der endoskopischen Diagnostik eine Einteilung vorzunehmen. Eine manometrische Bestimmung des unteren Ösophagussphinkters würde eine physiologische Definition der Kardia ermöglichen. Dieses Verfahren ist in der Praxis des Stagingprozesses bei Patienten die in diesem Bereich eine Neoplasie aufweisen allerdings nicht anwendbar. Während einer endoskopischen Untersuchung der Speiseröhre und des 
Magens wird versucht $\mathrm{zu}$ beurteilen, in welchem Bereich sich der Übergang von ösophagealem Plattenepithel zu gastralem Zylinderepithel befindet. Diese Beurteilung ist möglich, hängt jedoch von der Expertise des Untersuchers ab. Der Übergang wird als ZLinie bezeichnet und ist als Landmarke verwendbar. Als Richtlinie für die definitive Bestimmung der Kardia kann dieser Epithelübergang allerdings ebenfalls nicht zwingend verwendet werden, da die Z-Linie im Verlauf des Lebens nach proximal wandern kann. Dies geschieht vor allem bei Menschen mit gastroösophagealem Reflux. Somit wird heutzutage das proximale Ende der obersten Magenfalte als Beginn des Magens und somit als Ende der Speiseröhre angesehen (Stein et al. 2003).

Als gastroösophagealer Übergang wird der Bereich definiert, der sich von $5 \mathrm{~cm}$ oberhalb bis zu $5 \mathrm{~cm}$ unterhalb der Kardia erstreckt. In diesem Gebiet werden drei Typen von Adenokarzinomen unterschieden (Siewert et al. 1987):

- Typ-I: Adenokarzinom des distalen Ösophagus

- Typ-II: Kardiakarzinom

- Typ-III: Subkardiales Magenkarzinom

Tumoren des AEG-Typ-I nach Siewert befinden sich mit ihrer Hauptmasse im Bereich von $1 \mathrm{~cm}$ bis $5 \mathrm{~cm}$ oral des anatomischen gastroösophagealen Übergangs. Diese entwickeln sich auf der Basis einer bestehenden intestinalen Metaplasie (Barrett-Ösophagus). Die Hauptmasse von AEG-Karzinomen vom Typ-II nach Siewert liegt $1 \mathrm{~cm}$ oral bis $2 \mathrm{~cm}$ aboral des Übergangs von Ösophagus zu Magen. Ihre Entwicklung basiert häufig auf Kardiaepithel oder auf kurzen Bereichen mit einer intestinalen Metaplasie des ösophagogastralen Übergangs. Liegt der Tumor im Bereich von $2 \mathrm{~cm}$ bis $5 \mathrm{~cm}$ aboral der anatomischen Grenze, handelt es sich um ein subkardiales Magenkarzinom der Klassifikation AEG-Typ-III nach Siewert (Stein et al. 2000).

Die verschiedenen Typen der beschriebenen gastroösophagealen Übergangskarzinome kommen unterschiedlich häufig vor. In ihrer Veröffentlichung von 1987 stellten Siewert et al. die folgende Verteilung vor: von den Patienten ihres Kollektivs, die aufgrund eines Adenokarzinoms des gastroösophagealen Übergangs operiert wurden, wiesen 33,6\% einen Tumor vom AEG-Typ-I auf, bei 20,6\% der Patienten lag ein AEG-Typ-II-Tumor vor, und 45,8\% der Patienten zeigten einen Tumor vom AEG-Typ-III. Männer sind grundsätzlich deutlich häufiger betroffen als Frauen (Siewert et al. 1987).

\subsubsection{Diagnostik}

Als Standardverfahren zur Diagnosestellung wird von den Leitlinien die vollständige endoskopische Untersuchung von Speiseröhre und Magen aufgeführt. Dieses Verfahren besitzt die größte Spezifität sowie Sensitivität für den Tumornachweis im Bereich des oberen Gastrointestinaltrakts (Leitlinie Magenkarzinom 2012) und sollte daher frühzeitig zur Anwendung kommen. Die Entnahme von Biopsien aus den suspekten Arealen erfolgt zur histologischen Sicherung des Tumors. Durch die beschriebene mögliche Umwandlung 
des Epithels wird dieser diagnostische Prozess erschwert. In der Endoskopie stellt sich die Schleimhaut des Magens rötlich dar. Wenn es zu einem Ersatz des weißlichen Plattenepithels durch rote Schleimhaut kommt, so verlagert sich der Übergang von Weiß nach Rot, und diese Farbveränderung stellt nicht mehr den Übergang vom Ösophagus in den Magen dar.

\subsubsection{Therapie}

Bedingt durch das häufige Fehlen von Frühsymptomen kommt es bei vielen Patienten erst in einem fortgeschrittenen Tumorstadium zur Diagnosesicherung und zur Einleitung einer entsprechenden Therapie. Um die bestehenden therapeutischen Optionen optimal anwenden zu können, ist für den Patienten eine gezielte Diagnostik mit einem exakten Staging bedeutsam. Im interdisziplinären Team wird je nach vorliegendem Befund das therapeutische Vorgehen entschieden. Zur Orientierung dafür dient unter anderem die „S3-Leitlinie Diagnostik und Therapie der Plattenepithelkarzinome und Adenokarzinome des Ösophagus“ aus dem Jahr 2015 sowie die Leitlinie „Diagnostik und Therapie der Adenokarzinome des Magens und ösophagogastralen Übergangs“ aus dem Jahr 2012. Sofern der Patient operationsfähig und der Tumor chirurgisch resektabel ist, ergibt sich das weitere Vorgehen je nach klinischem Stadium. Standardmäßig wird bei der chirurgischen Resektion eines Ösophaguskarzinoms eine thorako-abdominale Ösophagusresektion mit Magenschlauch durchgeführt. Bei einem Patienten mit einem Magenkarzinom erfolgt eine Gastrektomie bzw. eine erweiterte Gastrektomie. Entsprechend der Lokalisation des Primärtumors wird entschieden, in welchem Ausmaß die ebenfalls durchzuführende Entfernung von Lymphknoten stattfindet. Standardmäßig handelt es sich hierbei um eine Zweifeld-Lymphadenektomie. Die separate Betrachtung der Adenokarzinome des gastroösophagealen Übergangs in Hinblick auf die Therapie hat sich etabliert, und auch international kommt es vermehrt zu einer Nutzung der AEG-Klassifikation (Bruns 2012). Entsprechend der hausinternen Vereinbarung des Tumorboards der Universitätsmedizin Göttingen (UMG) erfolgt in der Regel die Therapie des AEG-Typ-I-Tumors durch eine neoadjuvante Radiochemotherapie und im Verlauf dann eine Resektion des Ösophagus. Bei AEG-Typ-II und Typ-III-Tumoren erfolgt präoperativ eine Chemotherapie und im Anschluss eine Gastrektomie bzw. eine erweiterte Gastrektomie. Jeder Patient wird jedoch individuell besprochen, sodass es auch hier Abweichungen gibt. Für Patienten, die an der UMG operiert wurden, zeigen sich abweichende Behandlungskonzepte. Durch eine neoadjuvante Therapie werden bei Tumoren, die sich in einem fortgeschrittenen Stadium befinden, die Bedingungen für das Erzielen einer R0-Resektion optimiert (s.u. 2.2.5), und es kommt zu einer Verbesserung des Gesamtüberlebens (Bruns 2012). In Ausnahmefällen wird bei Patienten, bei denen Lymphknotenvergrößerungen vorliegen, die nicht als Metastasen gewertet werden, perioperativ eine Chemotherapie durchgeführt (vier Zyklen prä- sowie vier Zyklen postoperativ). Dies passiert auf der Grundlage, dass es bisher keine 
prospektiv randomisierten Studien gibt, die Chemo- und Radiochemotherapien miteinander verglichen haben.

\subsection{Zielsetzung der Arbeit}

Das Ziel der Arbeit ist es den Weg der Diagnostik bei Patienten, die aufgrund eines Ösophagus- oder Magenkarzinoms in der Abteilung für Allgemein- und Viszeralchirurgie der Universitätsmedizin Göttingen behandelt wurden, näher darzustellen. Neben der Erfassung klassischer Faktoren wie Alter, Geschlecht, Histologie und Therapie liegt der Schwerpunkt der Arbeit auf der Erfassung der Tumorlokalisation bzw. der Güte und Vollständigkeit der hierzu durchgeführten Untersuchungen. Die Fragestellung zielt darauf ab, inwieweit Kontrolluntersuchungen in der therapierenden Abteilung notwendig sind, um den standardisierten Therapieweg einzuhalten. Darüber hinaus soll versucht werden, die diagnostischen Befunde der durchgeführten Therapie bzw. dem histopathologischen Befund gegenüberzustellen. Somit soll zuletzt der Bedarf identifiziert werden, an welchen Stellen Standardisierungsbedarf besteht, um möglicherweise überflüssige Untersuchungen zu reduzieren. 


\section{Patienten und Methoden}

Für die retrospektive Arbeit wurde auf Daten des Göttinger Comprehensive Cancer Center (G-CCC) zurückgegriffen. Das Universitätskrebszentrum ist ein interdisziplinäres Zentrum der Krankenversorgung an der Universitätsmedizin Göttingen mit dem Ziel der Verbesserung der Behandlungsergebnisse und der Lebensqualität der Patienten mit bösartigen Tumorerkrankungen (G-CCC 2017b). Seit dem Jahr 1989 führt die Universitätsmedizin Göttingen ein klinisches Krebsregister. In diesem Register werden Daten über die Diagnose, die Tumorlokalisation und über den Verlauf der Behandlung nach den Vorschriften des Datenschutzes erfasst (G-CCC 2017a). Anhand einer Datenabfrage in diesem Krebsregister wurde eine Patientenliste erstellt. Die Suchkriterien waren entsprechend der Internationalen statistischen Klassifikation der Krankheiten und verwandter Gesundheitsprobleme die jeweiligen Diagnoseschlüssel für das Ösophaguskarzinom (ICD Code C15.- Bösartige Neubildung des Ösophagus) sowie für das Magenkarzinom (ICD Code C16.- Bösartige Neubildung des Magens) (ICD-10-GM 2016). Es wurden die Daten von 246 Patienten, die im Zeitraum von 2007 bis 2012 im Universitätsklinikum Göttingen in der Abteilung für Allgemein- und Viszeralchirurgie operiert wurden, ausgewertet.

\subsection{Datenerhebung}

Zur Erhebung der Daten wurden die Patientenakten im Zentralarchiv des Universitätsklinikums Göttingen eingesehen. Es wurden Informationen aus den in den Akten vorhandenen Dokumenten wie Arztbriefe, Anamnesebögen, Verlegungsberichte, Untersuchungsbefunde (z. B. von Ösophagogastroskopie, Computertomographie, Endosonographie), Operations- und Anästhesieprotokolle sowie Befundberichte aus der Pathologie gesammelt. Jeder Patient bekam zu Beginn der Datenerhebung eine Identifikationsnummer zur Pseudonymisierung, die nur den an der Auswertung beteiligten Personen bekannt war. Die Daten wurden nach Abschluss der Erfassung anonymisiert und die Analyse erfolgte mithilfe des Tabellenkalkulationsprogramms Microsoft ${ }^{\circledR}$ Excel ${ }^{\circledR}$ für Mac 2011.

\subsection{Patientendaten}

\subsubsection{Persönliche Daten, Risikofaktoren und ICD-Code}

Erfasst wurden persönliche Daten der Patienten wie das Geburtsdatum, Geschlecht, Körpergröße und -gewicht. Anhand des Geburtsdatums wurde das Alter der Patienten bei der Operation errechnet. Mithilfe von Körpergröße und -gewicht wurde der jeweilige 
Body-Mass-Index bestimmt. Dieser ist eine Kenngröße, um das Körpergewicht in Relation zur Körpergröße bewerten zu können. Des Weiteren wurden Informationen gesammelt über Risikofaktoren wie Tabak- und Alkoholkonsum sowie über eine mögliche Refluxsymptomatik und über das Vorliegen einer Dysphagie. Die jeweiligen ICD-Codes (International statistical classification of diseases and related health problems) wurden entsprechend der vierstelligen ausführlichen Systematik erfasst und in Untergruppen für das Ösophagus- und das Magenkarzinom aufgeteilt.

\subsection{2 Ösophagogastroskopie, Histologie und Endosonographie}

Aus den Unterlagen der Ösophagogastroskopie wurde das Datum der Untersuchung erfasst sowie von wem und wo (Ort bzw. Einrichtung) diese durchgeführt wurde. Anhand der endoskopischen Befunde wurde analysiert, ob bezüglich des möglichen Vorhandenseins einer Barrett-Mukosa eine Stellungnahme dokumentiert wurde, und wenn dies der Fall war, ob eine weitere Unterteilung in eine kurz- oder langstreckige Metaplasie vorgenommen wurde. Zur Bestimmung der Tumorlokalisation wurde erfasst, ob eine Angabe in Zentimeter ab Zahnreihe bezüglich Beginn und Ende des Tumors dokumentiert wurde bzw. ob eine im Freitext beschriebene Lokalisation des Tumors erfolgt ist. Des Weiteren wurde erfasst, ob eine Einteilung des endoskopisch sichtbaren Befundes entsprechend der Klassifikation nach Siewert erfolgt ist. Anhand der vorliegenden Befunde wurde analysiert, ob bei den Patienten eine Dokumentation über die anatomische Lage des gastroösophagealen Übergangs vorlag, also den Übergang von ösophagealem Plattenepithel zu gastralem Zylinderepithel. Dieser als Z-Linie bezeichnete Übergang dient als Landmarke und ist vor allem für die Einordnung der Tumorlokalisation von entscheidender Bedeutung. Es wurde ausgewertet, ob es zu einer Erwähnung der Z-Linie gekommen ist und ob die Lage mit einer Zentimeterangabe exakt beschrieben wurde. Zusätzlich wurde vermerkt, ob eine Passierbarkeit gegeben war oder ob gegebenenfalls der Befund einer tumorbedingten Stenose vorlag. In einem weiteren Schritt erfolgte anhand der vorhandenen Daten die Einteilung der Tumorlokalisation in oberen, mittleren und unteren Ösophagus sowie eine Lokalisation am gastroösophagealen Übergang oder im Magen. Für eine bei einigen Patienten durchgeführte zweite Ösophagogastroskopie wurden dieselben Daten erfasst. Gleiches erfolgte mit den Daten aus den Untersuchungsberichten der Endosonographie. Diese Untersuchung kommt neben der Endoskopie eine große diagnostische Bedeutung zu (Englisch-Fritz et al. 2008).

Für eine exakte Diagnosestellung wird während der endoskopischen Untersuchungen eine Gewebeprobe (Biopsie) entnommen. Die daraus resultierenden histopathologischen Befunde wurden erfasst. Hier erfolgte eine Dokumentation über das Datum der Biopsiebefundung sowie über die klinische Klassifikation der präoperativen histologischen Einteilung entsprechend den WHO-Kriterien (Hamilton et al. 2000):

- Adenokarzinom

- Plattenepithelkarzinom 
- andere (Spindelzellkarzinom, Kleinzellkarzinom, undifferenziertes Karzinom)

- nicht bekannt

Außerdem wurde die Graduierung des Tumors erfasst. Das Grading stuft den Malignitätsgrad des Tumors aufgrund histologischer und zytologischer Kriterien ein. Grad 1 (G1) steht für ein gut differenziertes Karzinom, Grad 2 (G2) beschreibt ein mäßig differenziertes Karzinom, ein schlecht differenziertes Karzinom wird mit Grad 3 (G3) beschrieben, und Grad 4 (G4) steht für ein undifferenziertes Karzinom (Böcker et al. 2008). Auch eine Einteilung des Tumors anhand der klinischen TNM-Klassifikation wurde im Verlauf des Stagingprozesses vorgenommen. Da zur präoperativen Stadieneinteilung der Ösophagus- und Magenkarzinome die Befunde der Endosonographie dienen, wurde der Klassifikation ein u-Symbol vorangestellt $(\mathrm{uT} / \mathrm{uN})$. Dies signalisiert, dass die Tumoreindringtiefe sowie eine mögliche Ausbreitung in benachbarte Lymphknoten mithilfe eines Ultraschallverfahres ermittelt wurden (Bösing et al. 2003). Die Eindringtiefe des Primärtumors, die Lymphknotenmetastasierung und die Fernmetastasierung wurden für das Ösophaguskarzinom sowie für das gastroösophageale Übergangskarzinom entsprechend den Richtlinien der UICC (Union internationale contre le cancer) wie folgt beschrieben (Wittekind et al. 2015):

TNM-Klassifikation des Ösophaguskarzinoms

T - Primärtumor

- Tx Primärtumor kann nicht beurteilt werden

- T0 Kein Anhalt für Primärtumor

- Tis Carcinoma in situ

- T1 Tumor infiltriert Lamina propria, Muscularis mucosae oder Submucosa

- T2 Tumor infiltriert Muscularis propria

- T3 Tumor infiltriert Adventitia

- T4 Tumor infiltriert Nachbarstrukturen

$\mathrm{N}$ - regionäre Lymphknoten

- Nx Regionäre Lymphknoten können nicht beurteilt werden

- N0 Keine regionären Lymphknotenmetastasen

- N1 Metastasen in 1 bis 2 regionären Lymphknoten

- N2 Metastasen in 3 bis 6 regionären Lymphknoten

- N3 Metastasen in 7 oder mehr regionären Lymphknoten

M - Fernmetastasen

- M0 Keine Fernmetastasen

- M1 Fernmetastasen

Tumoren mit einem Zentrum im Magen, welche in maximal $5 \mathrm{~cm}$ Abstand zum gastroösophealen Übergang liegen und in diesen Übergang hineinwachsen, werden ebenfalls entsprechend der Einteilung für Ösophaguskarzinome klassifiziert. 
Bei Magenkarzinomen gilt für die T-Klassifikation eine abweichende Einteilung. Die Stadieneinteilung für regionäre Lymphknoten und Fernmetastasen erfolgt nach den gleichen oben aufgeführten Kriterien.

TNM-Klassifikation des Magenkarzinoms

$\mathrm{T}$ - Primärtumor

- Tx Primärtumor kann nicht beurteilt werden

- T0 Kein Anhalt für Primärtumor

- Tis Carcinoma in situ: intraepithelialer Tumor ohne Infiltration der Lamina propria, hochgradige Dysplasie

- T1 Tumor infiltriert Lamina propria, Muscularis mucosae oder Submucosa

- T2 Tumor infiltriert Muscularis propria

- T3 Tumor infiltriert Subserosa

- T4 Tumor perforiert Serosa oder infiltriert benachbarte Strukturen

$\mathrm{N}$ - regionäre Lymphknoten

- Nx Regionäre Lymphknoten können nicht beurteilt werden

- N0 Keine regionären Lymphknotenmetastasen

- N1 Metastasen in 1 bis 2 regionären Lymphknoten

- N2 Metastasen in 3 bis 6 regionären Lymphknoten

- N3 Metastasen in 7 oder mehr regionären Lymphknoten

$\mathrm{M}-$ Fernmetastasen

- M0 Keine Fernmetastasen

- M1 Fernmetastasen

Magenkarzinome lassen sich zudem noch anhand der histologischen Klassifikation nach Laurén in zwei Hauptgruppen unterteilen, entweder in den intestinalen oder in den diffusen Typ (Meyer und Öfner 2008). Zusätzlich wird ein Mischtyp beschrieben (Baretton und Aust 2017). Diese Daten wurden ebenfalls erfasst, wenn sie in den Befunden der Pathologie aufgeführt waren.

\subsubsection{Neoadjuvante Chemo- und Strahlentherapie}

Für jeden Patienten aus dem Gesamtkollektiv wurde erfasst, ob eine neoadjuvante Chemotherapie, eine kombinierte Radiochemotherapie oder eine alleinige Strahlentherapie durchgeführt wurde. Diesbezüglich wurde der Zeitraum der Behandlung dokumentiert sowie die erfolgten Zyklen und das Schema bzw. die Art des verwendeten Chemotherapeutikums. Des Weiteren wurde die Dosis der Gesamtbestrahlung erfasst. Falls es bei den behandelten Patienten zu vorzeitigen Abbrüchen in der Durchführung der neoadjuvanten Behandlung gekommen ist, wurden diese dokumentiert und die entsprechenden Gründe erfasst. 


\subsubsection{Operation}

Für jeden Patienten wurde erfasst, ob eine Operation an der Universitätsmedizin Göttingen durchgeführt wurde. War dies der Fall wurden Operateur, Dauer und Art der Operation dokumentiert. Die Unterteilung erfolgte nach folgenden Operationsverfahren:

- Thorako-abdominale Ösophagusresektion mit Magenschlauch

- Gastrektomie mit D II Lymphadenektomie (LAE)

- Erweiterte Gastrektomie

- Sonstige Operationsverfahren

\subsubsection{Postoperative Histologie und Resektionsstatus}

Der durch die Pathologie angefertigte histologische Befund des Resektionspräparates wurde für jeden operierten Patienten aus dem Kollektiv begutachtet. Hier erfolgte zur genaueren Analyse die Dokumentation der Angaben des Befundes erneut mithilfe der Einteilung nach WHO-Kriterien. Bei Patienten mit einem Magenkarzinom wurde die Klassifikation nach Laurén dokumentiert. Die anatomische Ausbreitung des Tumors wurde anhand des pathologischen TNM-Stadiums erfasst. Außerdem wurde die Anzahl der untersuchten und die der befallenen Lymphknoten dokumentiert. Des Weiteren wurde aus diesen Werte das Verhältnis von befallenen zu untersuchten Lymphknoten errechnet. Ebenso wurde das Fehlen oder Vorhandensein eines Residualtumors (Resttumor) dokumentiert, hierfür dient die R-Klassifikation zur Beschreibung des vorliegenden Resektionsstatus. Die R-Klassifikation beschreibt im Einzelnen folgendes (Wittekind et al. 2015):

- RX: Vorhandensein von Residualtumor kann nicht beurteilt werden

- R0: Kein Residualtumor

- R1: Mikroskopischer Residualtumor

- R2: Makroskopischer Residualtumor

Die Ergebnisse der endosonographisch vorgenommenen TN-Klassifizierung wurden mit der postoperativen Einteilung verglichen. Es wurde dargestellt, ob es zu einer identischen Einordnung gekommen ist, oder ob die Ausbreitung des Tumors sowie der Lymphknotenstatus über- oder unterschätzt wurden.

\subsubsection{Adjuvante Chemo- und Strahlentherapie}

Über eine möglicherweise postoperativ durchgeführte adjuvante Chemotherapie, Bestrahlung oder Radiochemotherapie wurden dieselben Informationen dokumentiert wie über diese neoadjuvant durchgeführten Verfahren. Es wurde außerdem erfasst, ob bei den Patienten, bei denen es neoadjuvant zur Durchführung einer Chemotherapie gekommen war, diese Therapie postoperativ fortgeführt wurde. 


\subsection{Statistische Analyse}

Zur Datenerfassung diente das Tabellenkalkulationsprogramm Microsoft ${ }^{\circledR}$ Excel ${ }^{\circledR}$ für Mac 2011 in dem die entsprechenden Erhebungen gespeichert und statistisch ausgewertet wurden. Mithilfe des Programms Microsoft ${ }^{\circledR}$ Excel ${ }^{\circledR}$ für Mac 2011 wurden Tabellen, Abbildungen sowie Säulen- und Kreisdiagramme erstellt, um die jeweiligen Ergebnisse graphisch darzustellen, und für einzelne Merkmale wurden Minimal-, Maximal und Medianwert bestimmt. 


\section{Ergebnisse}

\subsection{Häufigkeitsverteilung des Patientenkollektivs}

\subsubsection{Diagnoseklassifikation nach ICD-Code}

Das Gesamtkollektivs umfasst 246 Patienten $(n=246)$. Hieraus ergeben sich mehrere Untergruppen. Bei 41,9\% der Patienten ( $\mathrm{n}=103)$ liegt laut ICD-Klassifikation eine bösartige Neubildung des Ösophagus vor, 58,1\% der Patienten ( $\mathrm{n}=143)$ zeigen eine bösartige Neubildung des Magens. Die folgende Tabelle zeigt die genauere Verteilung des Gesamtkollektivs in Bezug auf die Diagnosen, eingeteilt anhand der von der World Health Organization (WHO) herausgegebenen internationalen statistischen Klassifikation der Krankheiten und verwandter Gesundheitsprobleme.

Tabelle 1: Aufteilung des Gesamtkollektivs in Bezug auf die bestehende Diagnose anhand des ICD-Codes.

\begin{tabular}{llcc}
\hline ICD & Diagnose & Patientenzahl & Angaben in \% \\
\hline C15.- & Bösartige Neubildung des Ösophagus & $\mathbf{1 0 3}$ & $\mathbf{1 0 0}$ \\
\hline C15.1 & Thorakaler Ösophagus & 1 & 1 \\
C15.3 & Ösophagus, oberes Drittel & 2 & 1,9 \\
C15.4 & Ösophagus, mittleres Drittel & 18 & 17,5 \\
C15.5 & Ösophagus, unteres Drittel & 63 & 61,2 \\
C15.8 & Ösophagus, mehrere Teilbereiche überlappend & 4 & 3,9 \\
C15.9 & Ösophagus, nicht näher bezeichnet & 15 & 14,6 \\
\hline C16.- & Bösartige Neubildung des Magens & $\mathbf{1 4 3}$ & $\mathbf{1 0 0}$ \\
\hline C16.0 & Kardia & 55 & 38,5 \\
C16.1 & Fundus ventriculi & 5 & 3,5 \\
C16.2 & Corpus ventriculi & 10 & 7 \\
C16.3 & Antrum pyloricum & 15 & 10,5 \\
C16.4 & Pylorus & 2 & 1,4 \\
C16.5 & Kleine Kurvatur des Magens, nicht näher & & 4,2 \\
& bezeichnet & 6 & 1,4 \\
C16.6 & Große Kurvatur des Magens, nicht näher & & 3,5 \\
& bezeichnet & 2 & 30,1 \\
\hline C16.8 & Magen, mehrere Teilbereiche überlappend & 5 & \\
C16.9 & Magen, nicht näher bezeichnet & 43 & \\
\hline
\end{tabular}




\subsubsection{Geschlechterverteilung}

Bei der Betrachtung des Patientenkollektiv im Hinblick auf die Geschlechterverteilung zeigt sich, dass sich das Gesamtkollektiv in 66 weibliche (26,8\%) und 180 männliche Patienten $(73,2 \%)$ aufteilt. In der Untergruppe der Patienten mit einer bösartigen Neubildung des Ösophagus liegt ein Verhältnis von 14\% weiblichen zu 86\% männlichen Patienten vor. Die Gruppe der Patienten mit einer bösartigen Neubildung des Magens teilt sich auf in 36\% weibliche und 64\% männliche Patienten.

Abbildung 1: Geschlechterverteilung des Gesamtkollektivs sowie der beiden Untergruppen (Patienten mit bösartiger Neubildung des Ösophagus bzw. des Magens).

\begin{tabular}{|ccc|}
\hline $\begin{array}{c}\text { Gesamtkollektiv, } \\
\mathbf{n = 2 4 6}\end{array}$ & $\begin{array}{c}\text { Patienten mit } \\
\text { bösartiger } \\
\text { Neubildung des } \\
\text { Ösophagus, } \mathbf{n = 1 0 3}\end{array}$ & $\begin{array}{c}\text { Patienten mit } \\
\text { bösartiger } \\
\text { Neubildung des } \\
\text { Magens, } \mathbf{n}=\mathbf{1 4 3}\end{array}$ \\
\hline männlich - weiblich $_{73 \%}$ & & \\
\hline
\end{tabular}

\subsubsection{Altersverteilung}

In einem weiteren Schritt wurde die Verteilung des Alters der Patienten betrachtet. Der Median des Gesamtkollektivs liegt bei 66 Jahren. Die Altersspanne lag zwischen 22 und 86 Jahren. Das Durchschnittsalter aller Patientinnen und Patienten lag bei 64 Jahren. Bei differenzierter Analyse zeigt sich, das Männer mit 63 Jahren durchschnittlich gut vier Jahre jünger sind als Frauen, die im Durchschnitt bei der Behandlung des Ösophagus- bzw. Magenkarzinoms ein Alter von 67 Jahren haben. Im weiteren Verlauf erfolgte eine Alterseinteilung in Gruppen von Patienten bis 40 Jahre, Patienten zwischen 41 und 50 Jahren, Patienten zwischen 51 bis 60 Jahren, Patienten zwischen 61 und 70 Jahren, Patienten zwischen 71 und 80 und über 80-jährige Patienten. Mithilfe dieser Unterteilung nach Altersgruppen zeigte sich, dass im Gesamtkollektiv die Gruppe der 61 bis 70 Jährigen mit 32, 9\% den größten Anteil aufwies, kurz vor der Gruppe der 71 bis 80-jährigen Patienten mit 30,1\% (siehe Abbildung 2). Teilt man das Patientenkollektiv in Untergruppen, bestehend aus Patienten mit einem Ösophaguskarzinom und Patienten mit einem Magenkarzinom, und betrachtet dann die geschlechterspezifische Altersaufteilung, zeigt sich Folgendes: In der Gruppe der Patienten mit einem Ösophaguskarzinom liegt das Durchschnittsalter der Frauen bei 64 Jahren, die Altersspanne reicht von 47 bis 75 Jahren. Bei Männern liegt das Durchschnittsalter mit 62 Jahren leicht unter dem der Frauen, und die Altersspanne reicht von 42 bis 78 Jahren. In der Gruppe der Patienten mit einem Magenkarzinom sind die Frauen im Durchschnitt 68 Jahre alt mit einer Altersspanne von 
41 bis 86 Jahren. Männer in dieser Gruppe haben ein durchschnittliches Alter von 64 Jahren, der jüngste Patient dieser Gruppe hat ein Alter von 22 Jahren, der Älteste von 86 Jahren.

Abbildung 2: Altersverteilung des Gesamtkollektivs und der beiden Untergruppen (Patienten mit der Diagnose Ösophagus- bzw. Magenkarzinom).

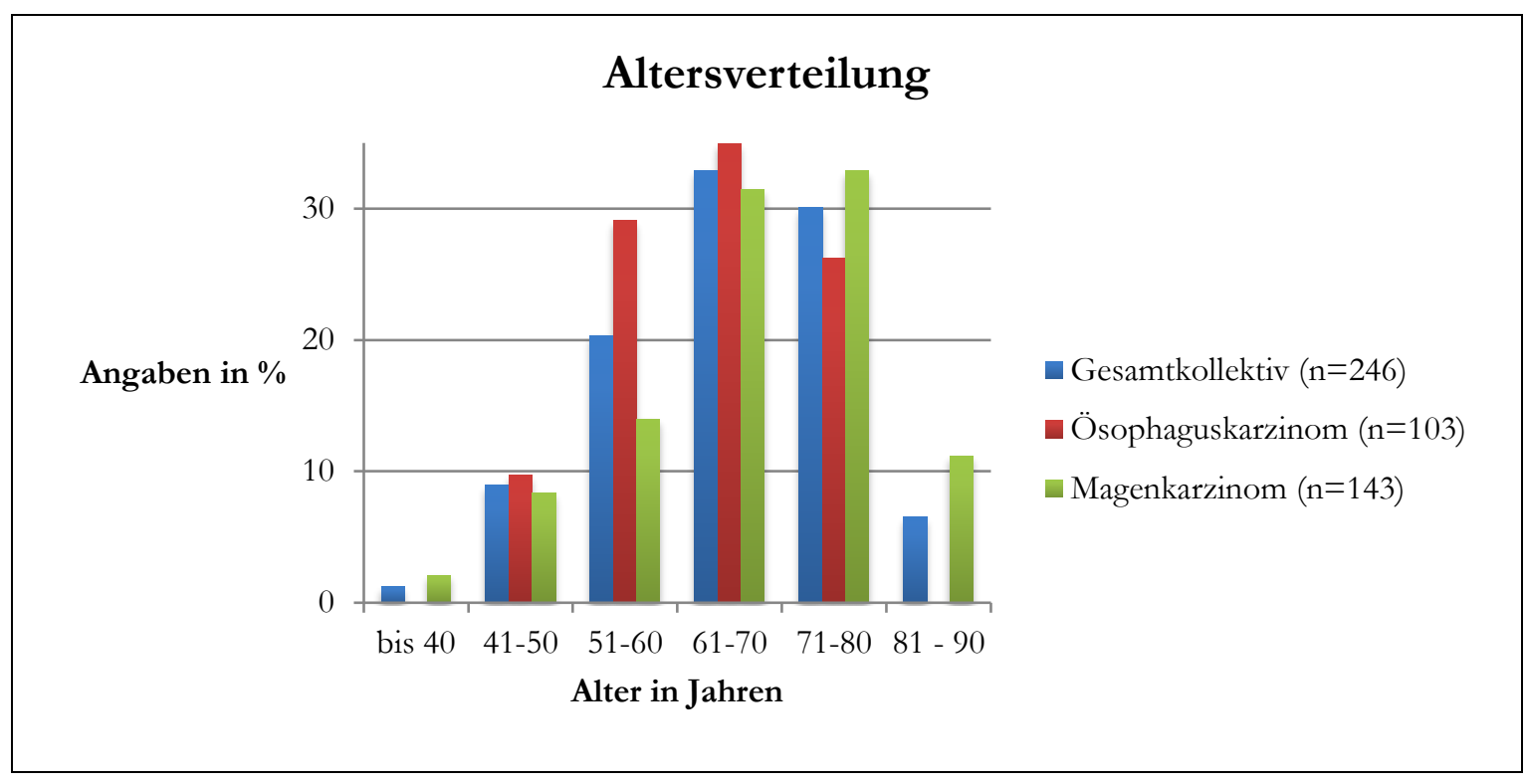

\subsubsection{Verteilung des Body-Mass-Index}

Es erfolgte eine Einteilung des Body-Mass-Index (BMI) nach Richtlinien der WHO in untergewichtig (BMI $<18,50 \mathrm{~kg} / \mathrm{m}^{2}$ ), normalgewichtig (BMI zwischen 18,50 kg/m $\mathrm{m}^{2}$ und $24,99 \mathrm{~kg} / \mathrm{m}^{2}$ ), präadipös (BMI $\geq 25 \mathrm{~kg} / \mathrm{m}^{2}$ bis $29,99 \mathrm{~kg} / \mathrm{m}^{2}$ ) und adipös (BMI über 30 $\mathrm{kg} / \mathrm{m}^{2}$ ) (World Health Organization 2000). Bei der Verteilung des Body-Mass-Index zeigte sich folgendes Bild: Im Gesamtkollektiv lag der BMI zwischen $16 \mathrm{~kg} / \mathrm{m}^{2}$ und $45 \mathrm{~kg} / \mathrm{m}^{2}$ mit einem Median von 26,3 kg/m². Mit 42\% ist der Anteil der übergewichtigen Patienten um einen Prozentpunkt höher als der Anteil der normalgewichtigen Patienten (41\%). Bei 2\% der Patienten lag der BMI unter 18,5 kg/m², womit es sich laut Definition der WHO um untergewichtige Patienten handelt. 15\% der Patienten wiesen einen Body-Mass-Index über $30 \mathrm{~kg} / \mathrm{m}^{2}$ auf. Somit handelte es sich bei dieser Gruppe um adipöse Patienten. Bei der separaten Betrachtung des Body-Mass-Index bei weiblichen und männlichen Patienten zeigte sich, dass der Median des BMI bei Frauen mit 24,8 kg/ $\mathrm{m}^{2}$ nur unwesentlich unter dem Median der Männer lag, welcher 26,4 kg/ $\mathrm{m}^{2}$ beträgt. In Abbildung 3 und 4 ist die Verteilung des Body-Mass-Index getrennt für Frauen und Männer graphisch dargestellt und zum Vergleich werden diesen Daten die Daten der Gesundheitsberichterstattung des Bundes über die Verteilung des Body-Mass-Index der erwachsenen Bevölkerung in der Bundesrepublik Deutschland von 2003/04 gegenübergestellt (Gesundheitsberichterstattung des Bundes 2017). 
Abbildung 3: Verteilung des Body-Mass-Index bei Frauen im Gesamtkollektiv und in den beiden Untergruppen (Patienten mit der Diagnose Ösophagus- bzw. Magenkarzinom) sowie der Verteilung in der Bundesrepublik Deutschland.

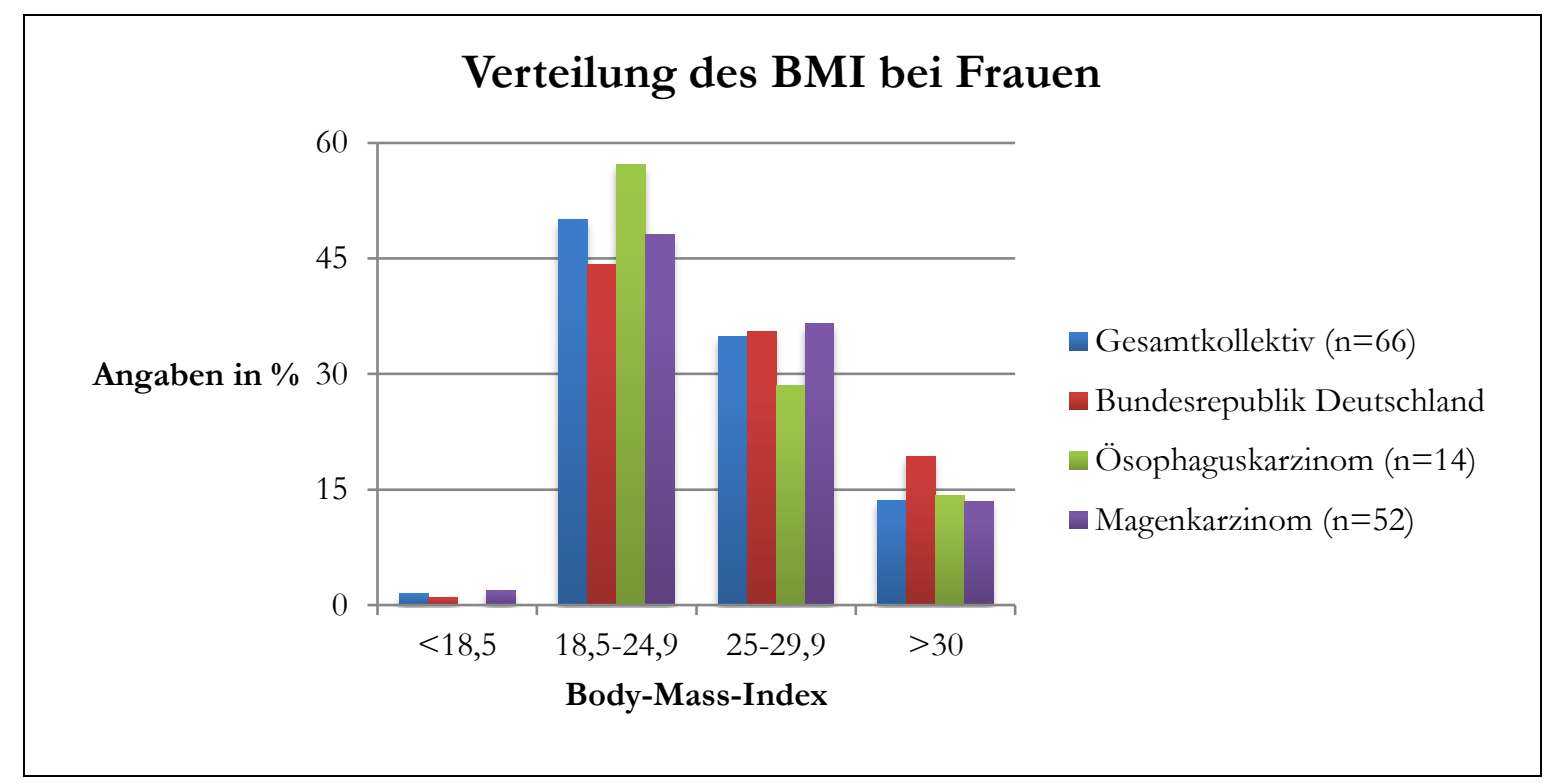

Abbildung 4: Verteilung des Body-Mass-Index bei Männern im Gesamtkollektiv und in den beiden Untergruppen (Patienten mit der Diagnose Ösophagus- bzw. Magenkarzinom) sowie der Verteilung in der Bundesrepublik Deutschland.

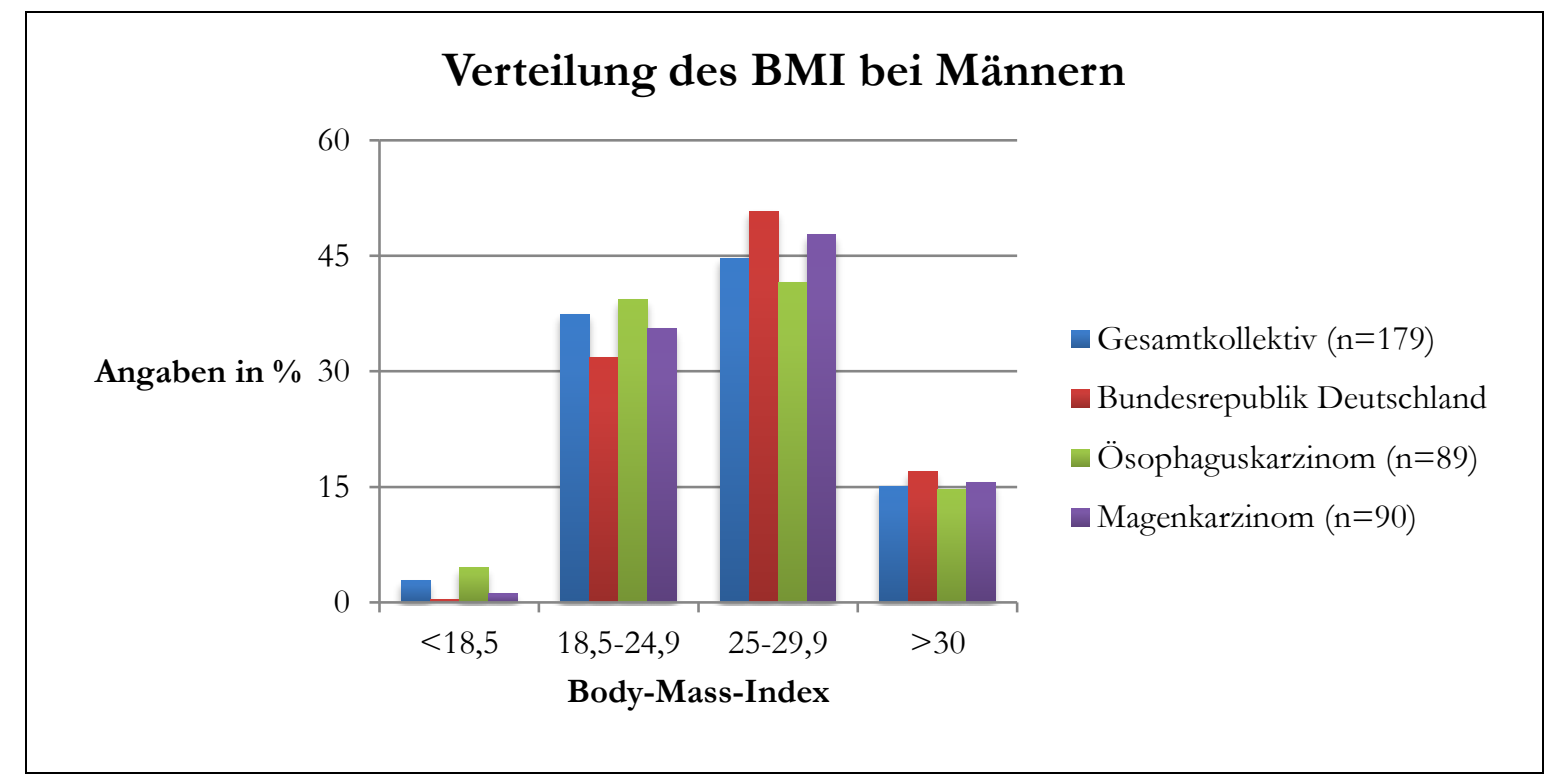

Bei genauerer Unterteilung der Patientengruppe mit einem Ösophaguskarzinom in Patienten mit einem Plattenepithelkarzinom und solche mit einem Adenokarzinom zeigt sich im Vergleich zu den Patienten mit einem Adenokarzinom des Magens die in Abbildung 5 dargestellte Verteilung des Body-Mass-Index. Patienten mit einem Plattenepithelkarzinom des Ösophagus haben bezogen auf den Body-Mass-Index einen 
Median von 24,1 kg/m $\mathrm{m}^{2}$ und zeigen somit einen niedrigeren Wert als Patienten mit einem Adenokarzinom des Ösophagus oder einem Magenkarzinom. Für die Gruppe der Patienten mit einem Adenokarzinom des Ösophagus liegt dieser Median bei 26,9 kg/m², für Patienten mit einem Magenkarzinom ergibt sich im vorliegenden Kollektiv ein Median von $26,5 \mathrm{~kg} / \mathrm{m}^{2}$.

Abbildung 5: Verteilung des Body-Mass-Index in den jeweiligen Gruppen der Patienten mit einem Plattenepithel- bzw. Adenokarzinom.

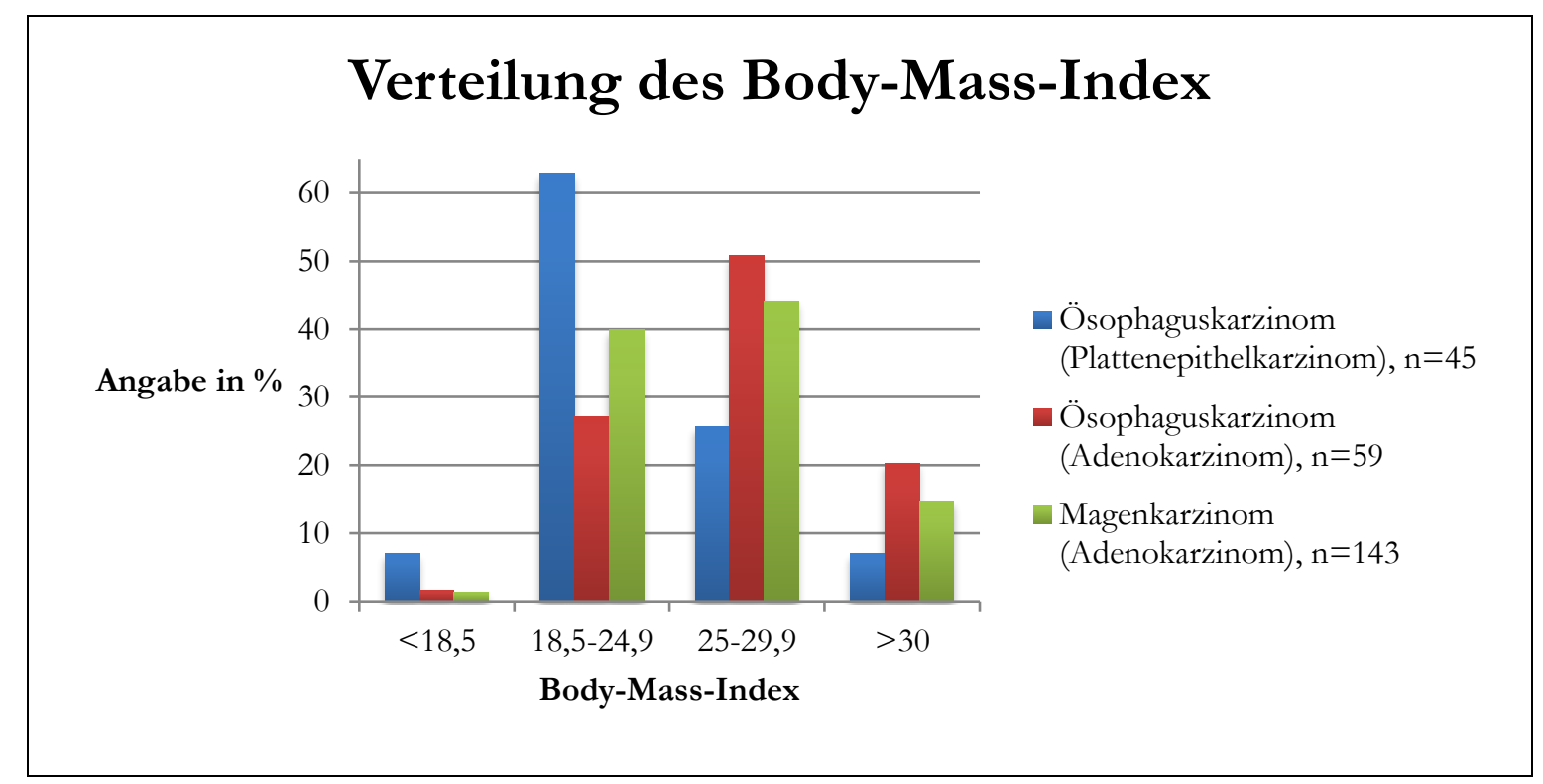

\subsubsection{Vorkommen der Risikofaktoren}

Der Anteil der Patienten, die einen Nikotinabusus in der Anamnese angaben, liegt insgesamt bei 36\%. Im Gesamtkollektiv gaben 15\% der Patienten an regelmäßig Alkohol zu konsumieren. Bei 25\% aller Patienten war ein Reflux bekannt und bei 15\% der Patienten des Gesamtkollektivs konnte endoskopisch ein Barrett-Ösophagus nachgewiesen werden. Symptome der Dysphagie wurden von insgesamt 37\% der Patienten angegeben. In Abbildung 5 und 6 ist entsprechend den Untergruppen für Ösophagus- und Magenkarzinompatienten das Vorkommen dieser Risikofaktoren graphisch dargestellt. 
Abbildung 6: Vorkommen von einigen Risikofaktoren im Kollektiv der Patienten mit einem Ösophaguskarzinom ( $\mathrm{n}=103)$.

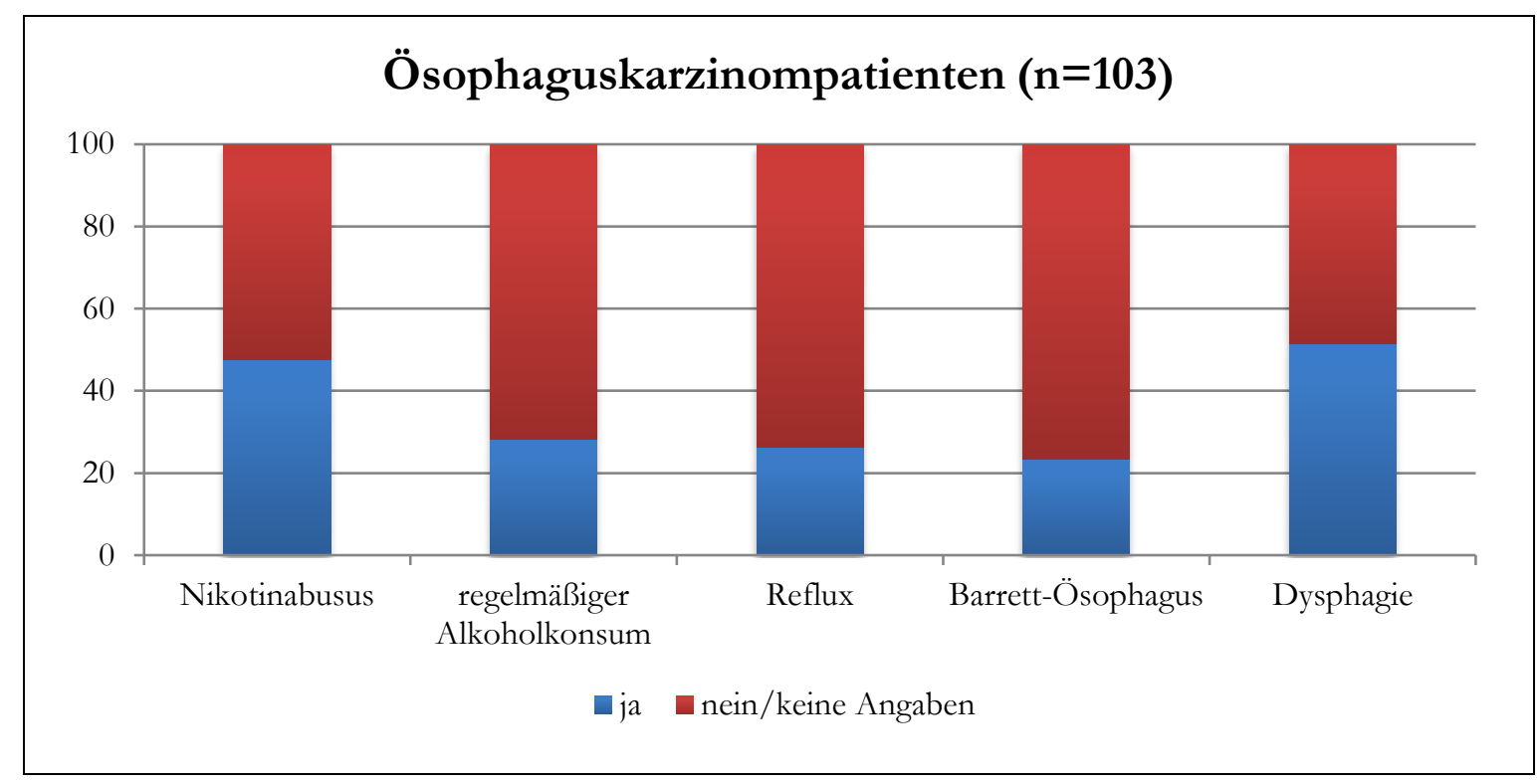

Abbildung 7: Vorkommen von einigen Risikofaktoren im Kollektiv der Patienten mit einem Magenkarzinom ( $\mathrm{n}=143)$.

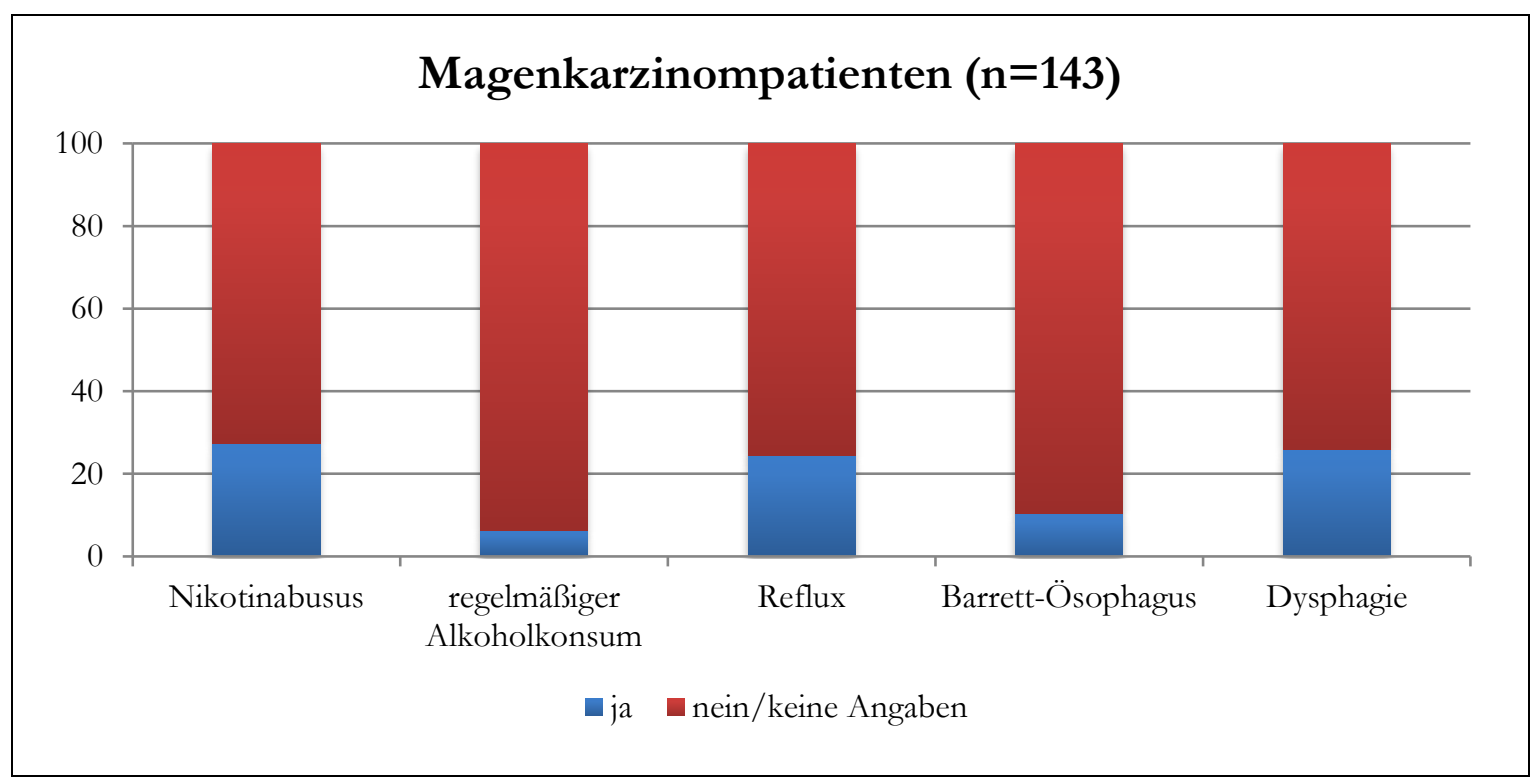

Bei 10\% der Patienten des Gesamtkollektivs konnte anhand der Daten ermittelt werden, dass sowohl Nikotin als auch Alkohol regelmäßig konsumiert werden. Insgesamt 71\% der Patienten zeigen mindestens einen der aufgeführten Risikofaktoren in der Anamnese, bei 29\% der Patienten des Gesamtkollektivs sind keine Risikofaktoren dokumentiert. 


\section{2 Ösophagogastroskopie}

Im Rahmen des Stagingprozesses kam es bei 234 Patienten des Kollektivs zur Durchführung einer Ösophagogastroskopie (97,5\%). Für die fehlenden 12 Patienten (2,5\%) existierten in den Akten keine Dokumente über die Durchführung dieser Maßnahme. Es ist davon auszugehen, dass die Untersuchung bei ihnen durchgeführt wurde, es jedoch nicht zu einer Archivierung der Befunde gekommen ist. Da diese somit nicht in die Auswertung einbezogen werden können, ergibt sich für die folgende Analyse $n=234$. Eine zweite Endoskopie wurde in 136 Fällen durchgeführt. Es kam also bei 58,1\% der Patienten zu einer Wiederholung dieser diagnostischen Maßnahme.

\subsubsection{Untersuchungsorte}

Anhand der in den Akten vorliegenden Befunde ließen sich 117 unterschiedliche Untersucher bzw. medizinische Einrichtungen festmachen, die an der Durchführung einer Ösophagogastroskopie beteiligt waren. Bei der Zusammenfassung dieser Daten hat sich das in Abbildung 7 dargestellte Bild ergeben. Somit wurden 15\% der ersten Endoskopien an der Universitätsmedizin Göttingen durchgeführt. Weitere $22 \%$ fanden außerhalb der Universitätsklinik in Göttingen statt. 59\% der Untersuchungen wurden außerhalb von Göttingen durchgeführt und in $4 \%$ der Fälle ist nicht bekannt, wo die Ösophagogastroskopie stattgefunden hat. Demnach wurde mit $85 \%$ bei einem Großteil der Patienten die erste Ösophagogastroskopie nicht am später operierenden Zentrum durchgeführt. In der Folge des Staging- und Therapieplanungsprozesses kam es bei 58,1\% der Patienten zu einer Wiederholung der Endoskopie. Hier zeigt die Verteilung, dass 55\% dieser zweiten Ösophagogastroskopie an der UMG durchgeführt wurden. Weitere 24\% der Untersuchungen fanden in anderen Einrichtungen in Göttingen statt, 17\% außerhalb von Göttingen und bei 4\% ist der Untersuchungsort unbekannt. Bei 10\% der Patienten, bei denen zwei Endoskopien durchgeführt wurden, fand sowohl die erste als auch die zweite Untersuchung an der Universitätsmedizin Göttingen statt. 
Abbildung 8: Graphische Darstellung der unterschiedlichen Untersuchungsorte für die erste Ösophagogastroskopie ( $\mathrm{n}=234)$.

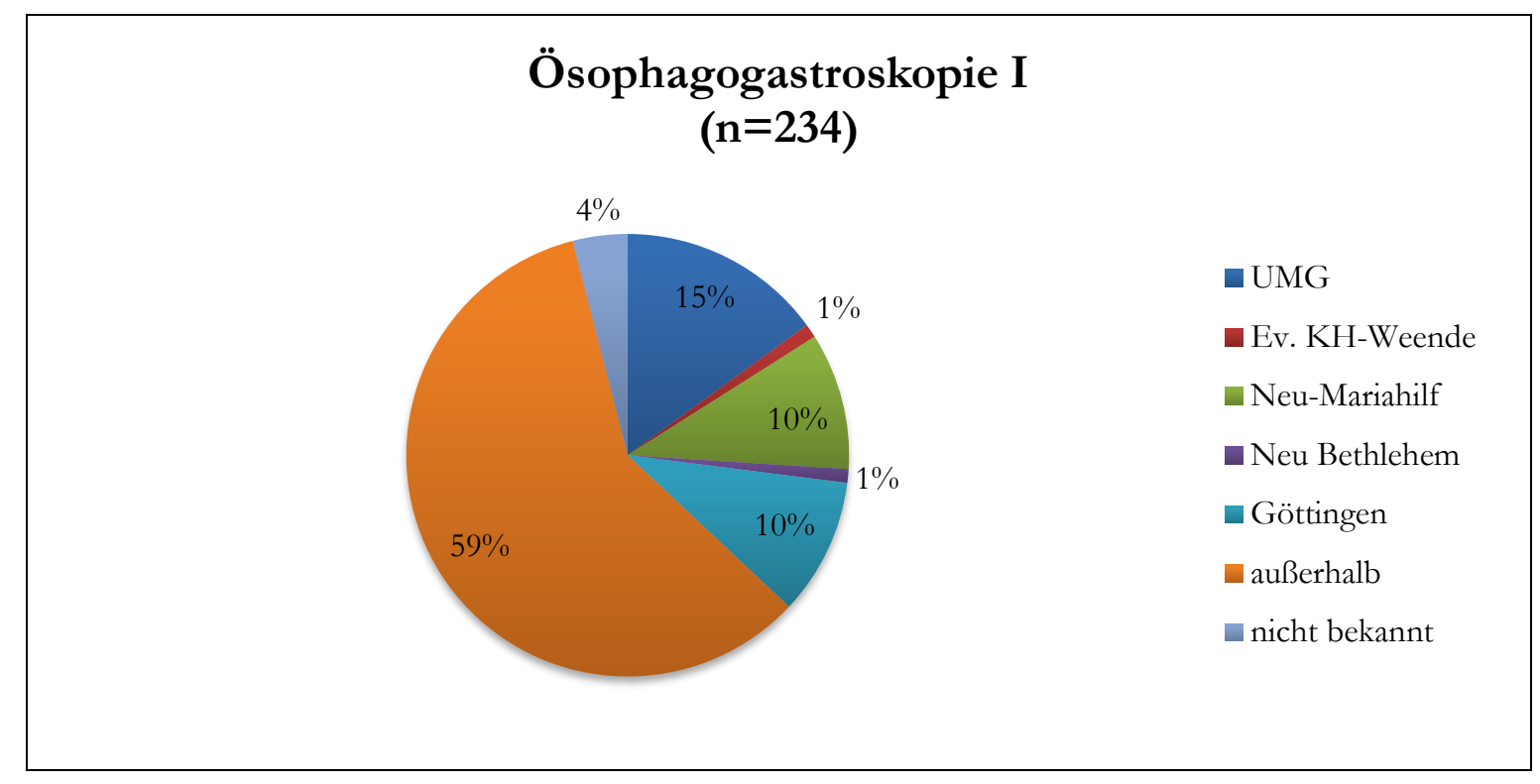

Abbildung 9: Graphische Darstellung der unterschiedlichen Untersuchungsorte für die zweite Ösophagogastroskopie ( $\mathrm{n}=136)$.

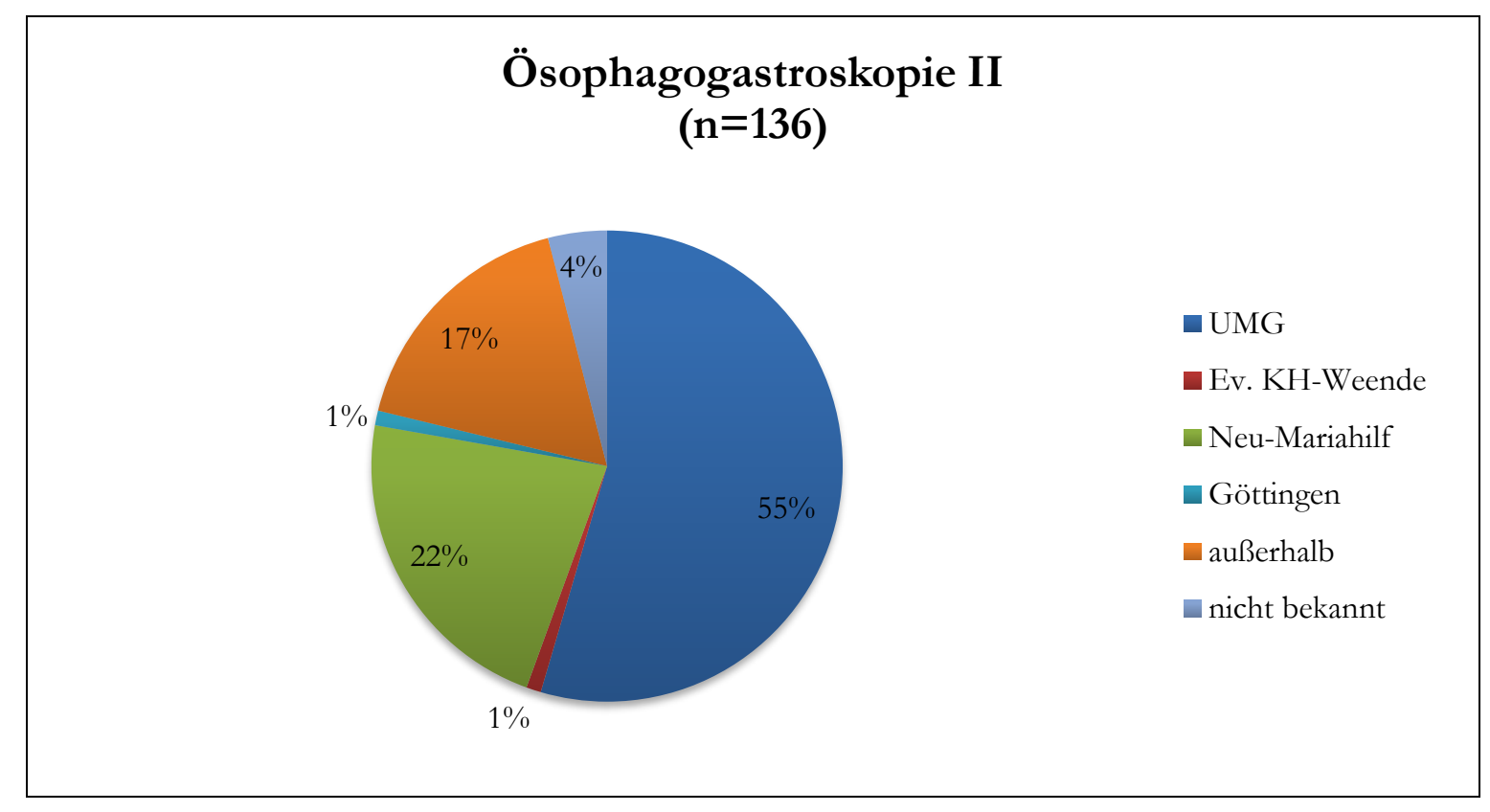




\subsubsection{Tumorlokalisation}

Die Aussagen bezüglich der Lokalisation einer Neoplasie fanden sich in den Befundberichten sowohl in Form von Zentimeterangaben die den Beginn und das Ende des Tumors in Zentimetern ab Zahnreihe dokumentieren, als auch in schriftlicher Form im Freitext angegeben. Für die graphische Darstellung der Verteilung der vorliegenden Tumorlokalisationen in Abbildung 6 wurden diese Informationen in die drei Kategorien Ösophagus, Magen und Übergang zusammengefasst.

Abbildung 10: Verteilung der vorliegenden Tumorlokalisationen entsprechend den Befunden von Endoskopie I ( $\mathrm{n}=234)$ und Endoskopie II ( $\mathrm{n}=136)$.

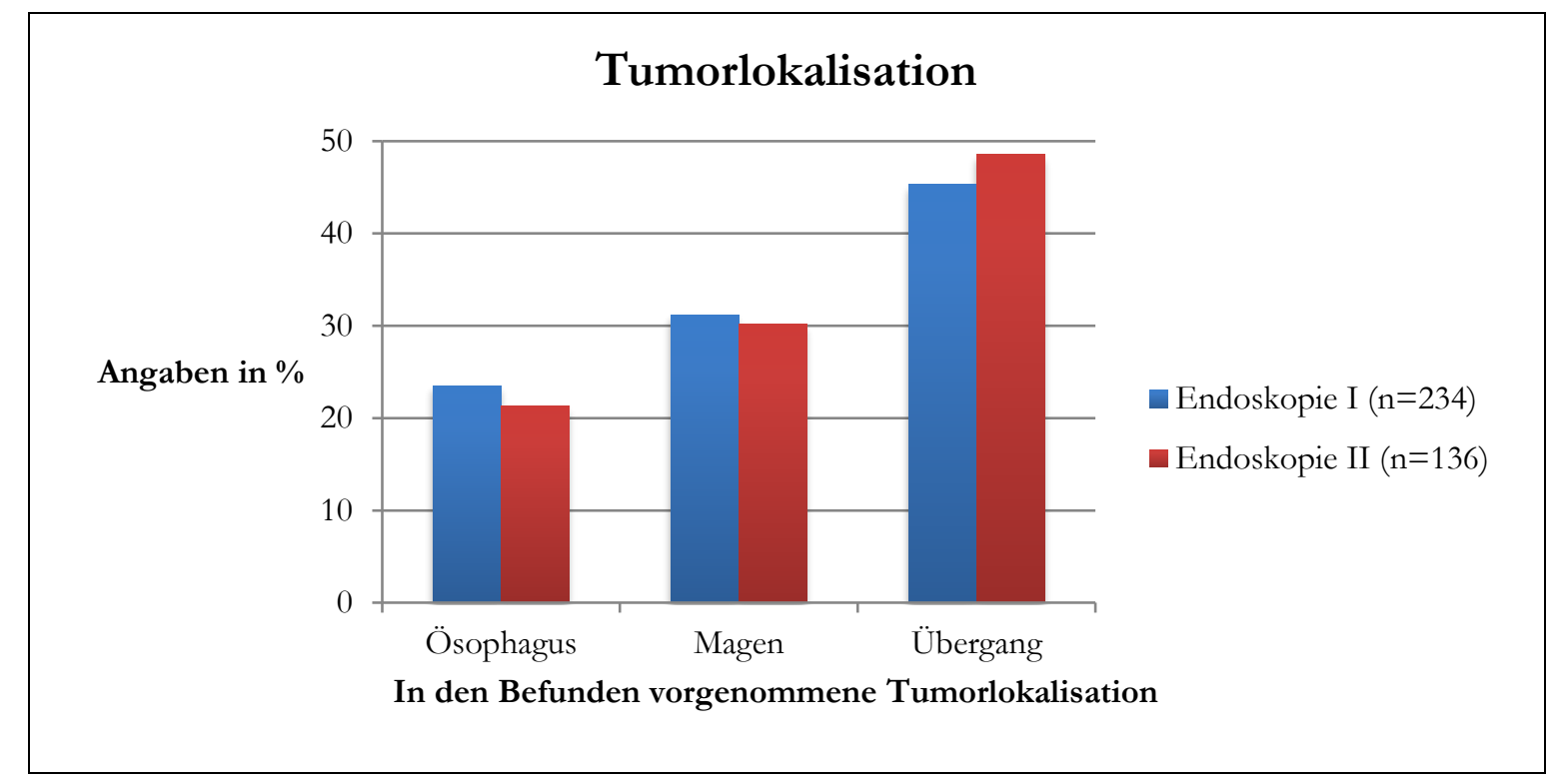

\subsubsection{Angaben zur Lokalisation der Z-Linie}

Bei der Auswertung der Gastroskopiebefunde wurde die Dokumentation bezüglich einer Angabe zur Lokalisation der Z-Linie mit der Aufzeichnung einer Zentimeterangabe genauer analysiert. Hierfür wurde das Gesamtkollektiv weiter unterteilt, zum einen in eine Patientengruppe, bei der die Ösophagogastroskopie an der Universitätsmedizin Göttingen durchgeführt wurde, und zum anderen in eine Gruppe, in der die Patienten außerhalb der UMG untersucht worden sind. Es zeigt sich folgendes Bild: Bei den 35 an der UMG durchgeführten Erstuntersuchungen wurde die Z-Linie in fünf Fällen beschrieben (14,3\%), in 199 Fällen fand die Ösophagogastroskopie in einer anderen Institution statt, die Z-Linie wurde bei diesen Untersuchungen insgesamt 35 mal dokumentiert (17,6\%). Bei der zweiten Untersuchung zeigte sich, dass bei 27 der 73 an der UMG durchgeführten Untersuchungen eine Dokumentation der Z-Linie erfolgte (37\%). Ein ähnlicher Wert ergibt sich auch bei der Analyse der nicht an der UMG durchgeführten zweiten Ösophagogastroskopie. Hier wurde bei 23 der insgesamt 63 Untersuchungen die Z-Linie im Befundbericht dokumentiert $(36,5 \%)$. 
Abbildung 11: Dokumentation der Z-Linie als Zentimeterangabe nach der ersten Ösophagogastroskopie an der Universitätsmedizin Göttingen sowie außerhalb.

\section{Universitätsmedizin Göttingen}

$(n=35)$

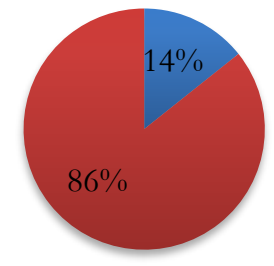

Z-Linie angegeben

Z-Linie nicht angegeben außerhalb

$(n=199)$

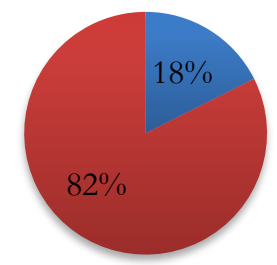

Z-Linie angegeben

Z-Linie nicht angegeben

Abbildung 12: Dokumentation der Z-Linie als Zentimeterangabe nach der zweiten Ösophagogastroskopie an der Universitätsmedizin Göttingen sowie außerhalb.

Universitätsmedizin Göttingen $(n=73)$

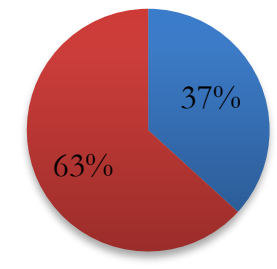

Z-Linie angegeben

$\square$ Z-Linie nicht angegeben außerhalb

$(n=63)$

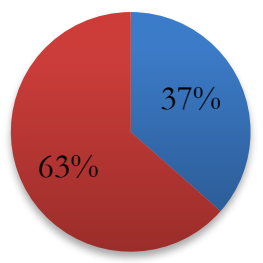

Z-Linie angegeben

Z-Linie nicht angegeben

\subsubsection{Angaben zur Barrett-Mukosa}

Anhand der Befundberichte aus der Endoskopie wurde ausgewertet, ob es zu einer Dokumentation bezüglich eines möglichen Vorliegens einer Barrett-Mukosa gekommen ist. Diese ist als mögliche Präkanzerose, vor allem der AEG-Typ-I-Tumorn (Stein et al. 2013) ein wichtiger diagnostischer Faktor. In den Unterlagen der ersten Ösophagogastroskopie wird eine solche Barrett-Mukosa in 34 der 234 Befunde beschrieben (14,5\%) (siehe Abbildung 11). Eine genauere Unterteilung dieser Metaplasie in kurz- $(<3 \mathrm{~cm})$ oder langstreckig $(>3 \mathrm{~cm})$ fand nur in 3,4\% der Fällen statt. 1,7\% der Patienten wiesen eine kurzstreckige und weitere 1,7\% eine langstreckige Barrett-Mukosa auf. Eine zweite Ösophagogastroskopie wurde bei 136 Patienten durchgeführt. Eine sichtbare Schleimhautveränderung im Sinne einer Barrett-Mukosa wurde bei 11\% der Patienten beschrieben. Eine weiterführende Einteilung fand in 3,7\% der Fälle statt. 2,9\% dieser Patienten zeigten eine kurzstreckige Barrett-Mukosa, und in $0,8 \%$ der Fälle lag bei dem Patient eine langstreckige Veränderung vor. Etwa zu gleichen Teilen wurde bei den übrigen 
Patienten das Vorliegen einer Barrett-Mukosa verneint bzw. es wurde keine entsprechende Angabe dokumentiert.

Abbildung 13: Verteilung der vorgenommenen Angaben bezüglich eines möglichen Vorliegens einer Barrett-Mukosa für Endoskopie I ( $n=234)$ und Endoskopie II ( $n=136)$.

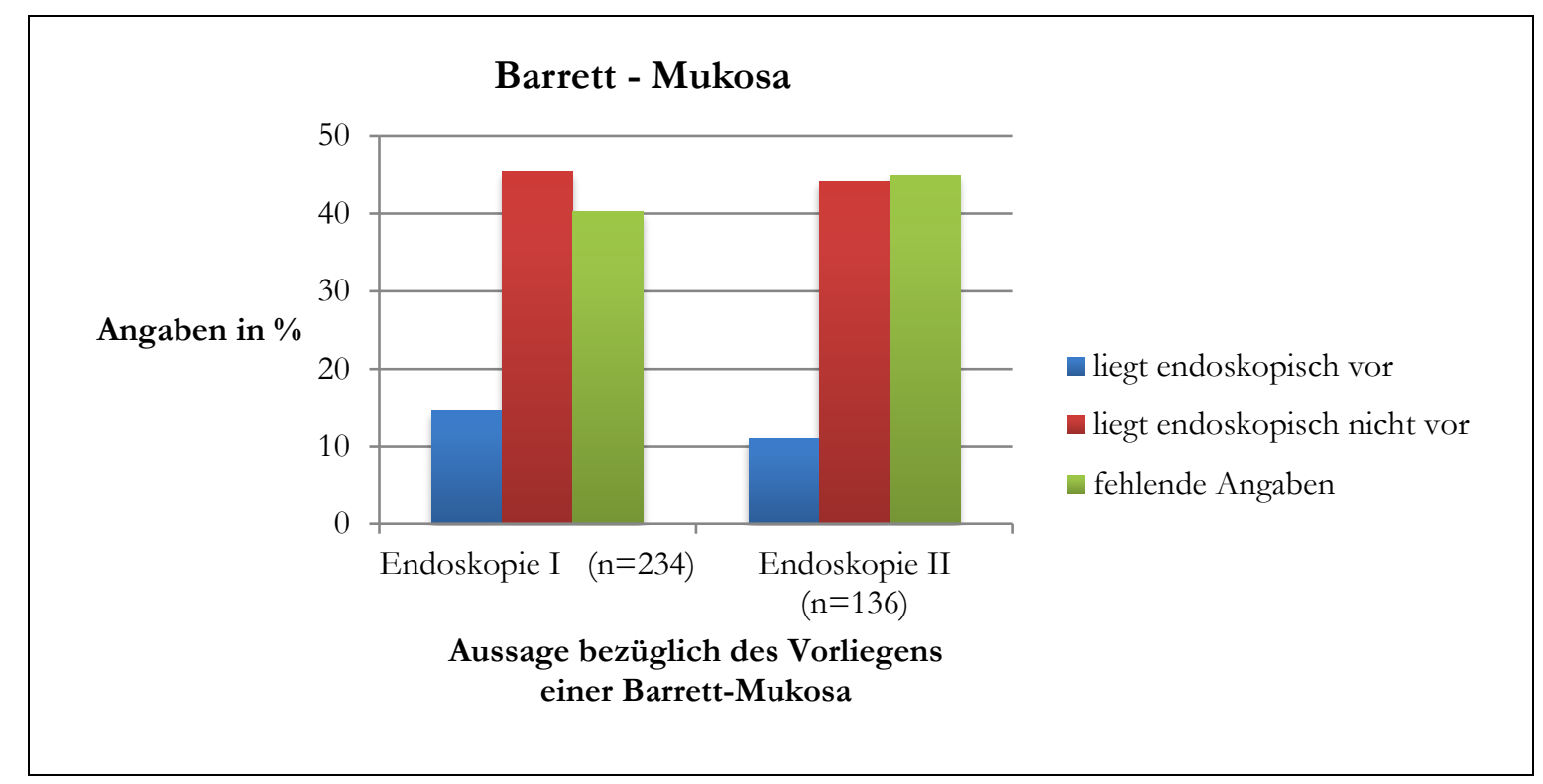

Eine Barrett-Mukosa wird vor allem bei Patienten mit einem Adenokarzinom im distalen Ösophagus erwartet, da diese die Präkanzerose darstellt. Eine Neoplasie in diesem Bereich liegt laut ICD-Klassifikation bei 63 Patienten vor. Nur bei 25,4\% dieser Patienten wird endoskopisch das Vorliegen einer Barrett-Mukosa diagnostiziert, in 33,3\% der Fälle wird das Vorliegen dieser intestinalen Metaplasie verneint und bei 41,3\% der Patienten befinden sich diesbezüglich keine Angaben in den Befundberichten. Im untersuchten Kollektiv wurde diese Schleimhautveränderung somit auch bei Patienten diagnostiziert die beispielsweise eine Neoplasie des mittlere Ösophagus oder des Magens aufweisen. Die Patienten, bei denen die Beschreibung einer Barrett-Mukosa vorliegt, wurden entsprechend der für sie zutreffenden ICD-Klassifikation (siehe Tabelle 1) in Gruppen eingeteilt. In Abbildung 14 und 15 werden die Ergebnisse der ersten und zweiten Endoskopie dargestellt. 
Abbildung 14: Verteilung der in Endoskopie I vorliegenden Barrett-Mukosa-Befunde für die Patienten entsprechend der für sie zutreffenden ICD-Klassifikation ( $\mathrm{n}=34)$.

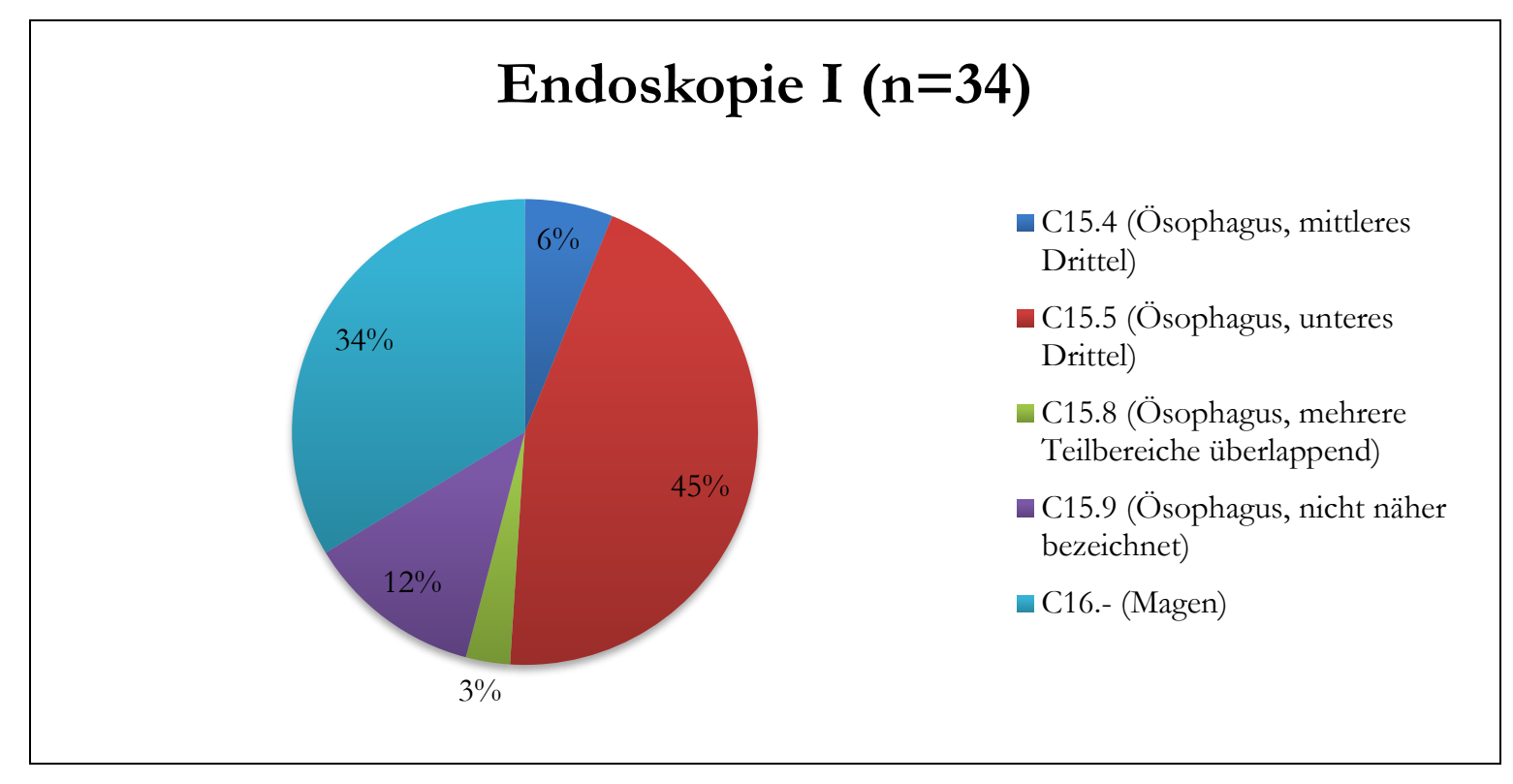

Abbildung 15: Verteilung der in Endoskopie II vorliegenden Barrett-Mukosa-Befunde für die Patienten entsprechend der für sie zutreffenden ICD-Klassifikation ( $\mathrm{n}=15)$.

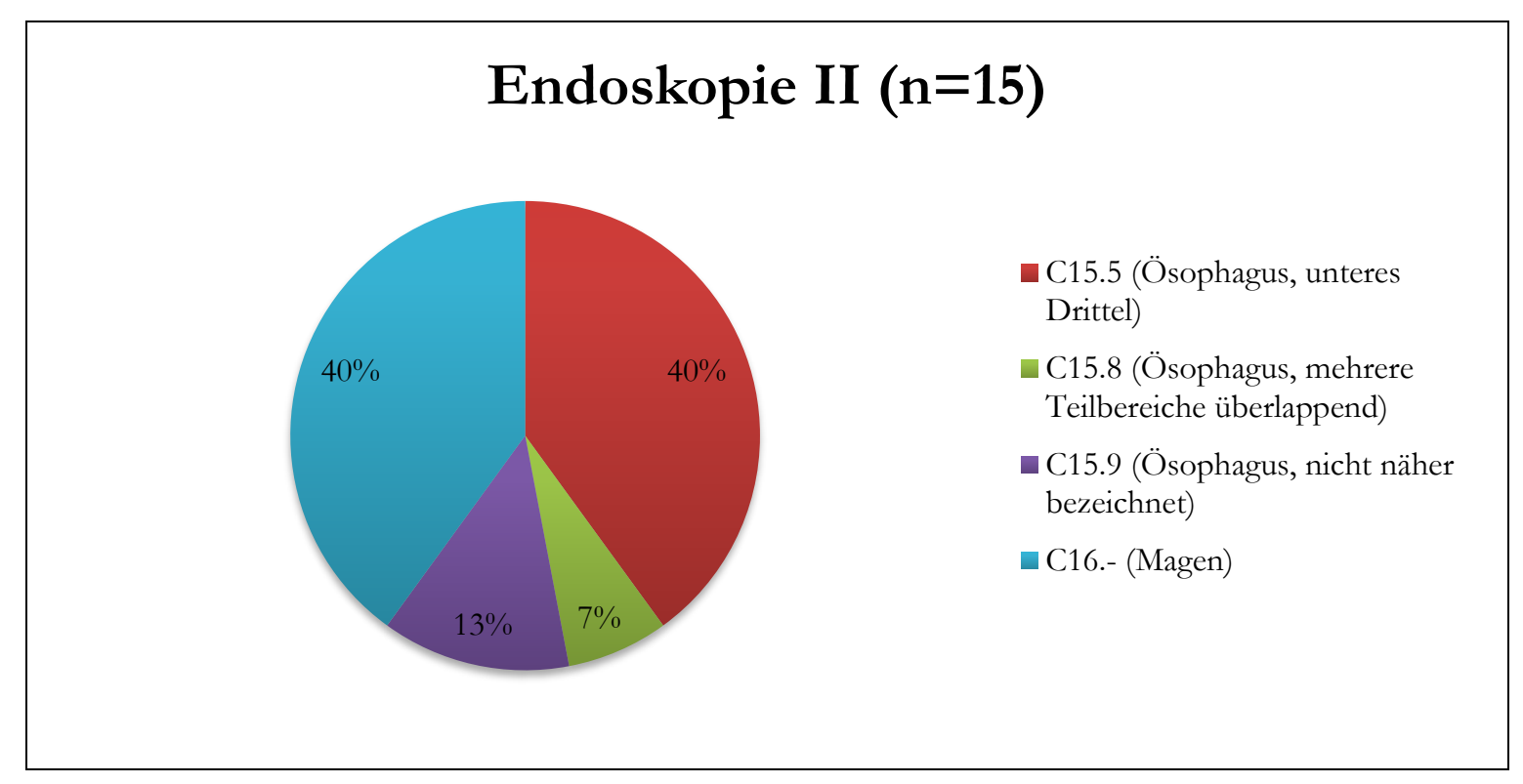




\subsubsection{Histologische Auswertung der in der Ösophagogastroskopie gewonnenen Biopsie}

Die histologische Auswertung der während der Ösophagogastroskopie gewonnenen Biopsien wurde auf Grundlage der Definitionen der World Health Organization getätigt, bei Magenkarzinompatienten wurde zusätzlich die Laurén-Klassifikation angewandt. Des Weiteren wurde der Differenzierungsgrad (Abkürzung als: „G“) angegeben. Bei der Erfassung der histologischen Daten spielt in der folgenden Berechnung keine Rolle, ob die Biopsie während der ersten oder der zweiten Ösophagogastroskopie gewonnen wurde. Es zeigte sich, dass in 223 Fällen (95,3\%) eine Einteilung nach Definition der WHO erfolgt ist, eine Klassifikation nach Laurén lag bei 80 Patienten (55,9\%) der Patienten mit einem Magenkarzinom vor. Die erhobenen Differenzierungsgrade zeigten folgendes Bild: Neun Patienten (3,9\%) wiesen den Differenzierungsgrad G1 auf, ein Nachweis für den Differenzierungsgrad G2 lag bei 79 Patienten (33,8\%) vor, eine Zuordnung zum Differenzierungsgrad G3 fand sich bei 62 Patienten (26,5\%). Der Differenzierungsgrad G4 wurde bei vier Patienten $(1,7 \%)$ beschrieben. Eine fehlende Zuordnung lag bei 80 Patienten $(34,2 \%)$ vor.

Abbildung 16: Verteilung der präoperativen Differenzierungsgrade im Gesamtkollektiv $(n=243)$.

\section{Differenzierungsgrad $(n=234)$}

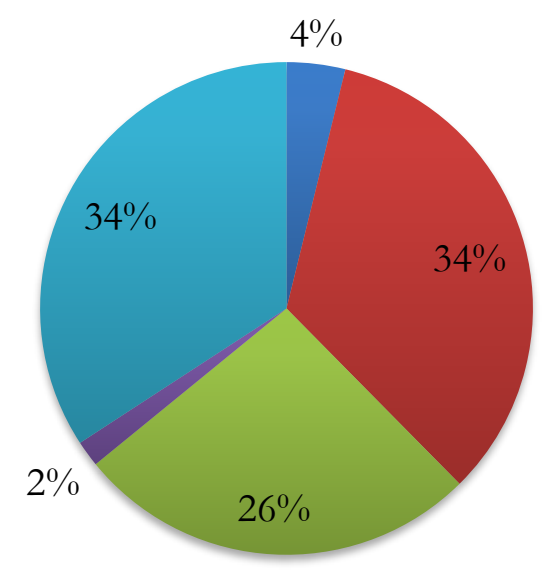

G1 $(3,85 \%)$

- G2 $(33,75 \%)$

- G3 $(26,5 \%)$

- G4 $(1,7 \%)$

veine Angaben (34,2\%)

\subsubsection{AEG-Klassifikation nach Siewert}

Die in der Endoskopie vorgenommene Einteilung zur Lokalisation der Neoplasie zeigt, dass bei 45,3\% der Patienten im Rahmen der ersten Ösophagogastroskopie der Tumor nicht klar als im Ösophagus oder Magen befindlich beschrieben wurde, bei einer zweiten Untersuchung war dies bei 48,5\% der Patienten der Fall. Bei diesem Patientenkollektiv liegt also ein Tumor vor, der sich im Bereich des gastroösophagealen Übergangs befindet. Für diese Tumoren ist eine Einteilung anhand der AEG-Klassifikation nach Siewert möglich. 
Die Auswertung der Endoskopiebefunde zeigt, dass in der ersten Untersuchung in insgesamt 21,7\% der Befunde eine solche Einteilung dokumentiert wurde. Bei einer zweiten endoskopischen Untersuchung enthielten die Unterlagen von 40,9\% der Patienten eine Dokumentation der AEG-Klassifikation nach Siewert (siehe Abbildung 17). Die genaue Verteilung ist in Abbildung 18 dargestellt.

Abbildung 17: Verteilung der vorgenommenen Angaben bezüglich einer möglichen Einteilung entsprechend der Siewert-Klassifikation nach erster und zweiter Ösophagogastroskopie.

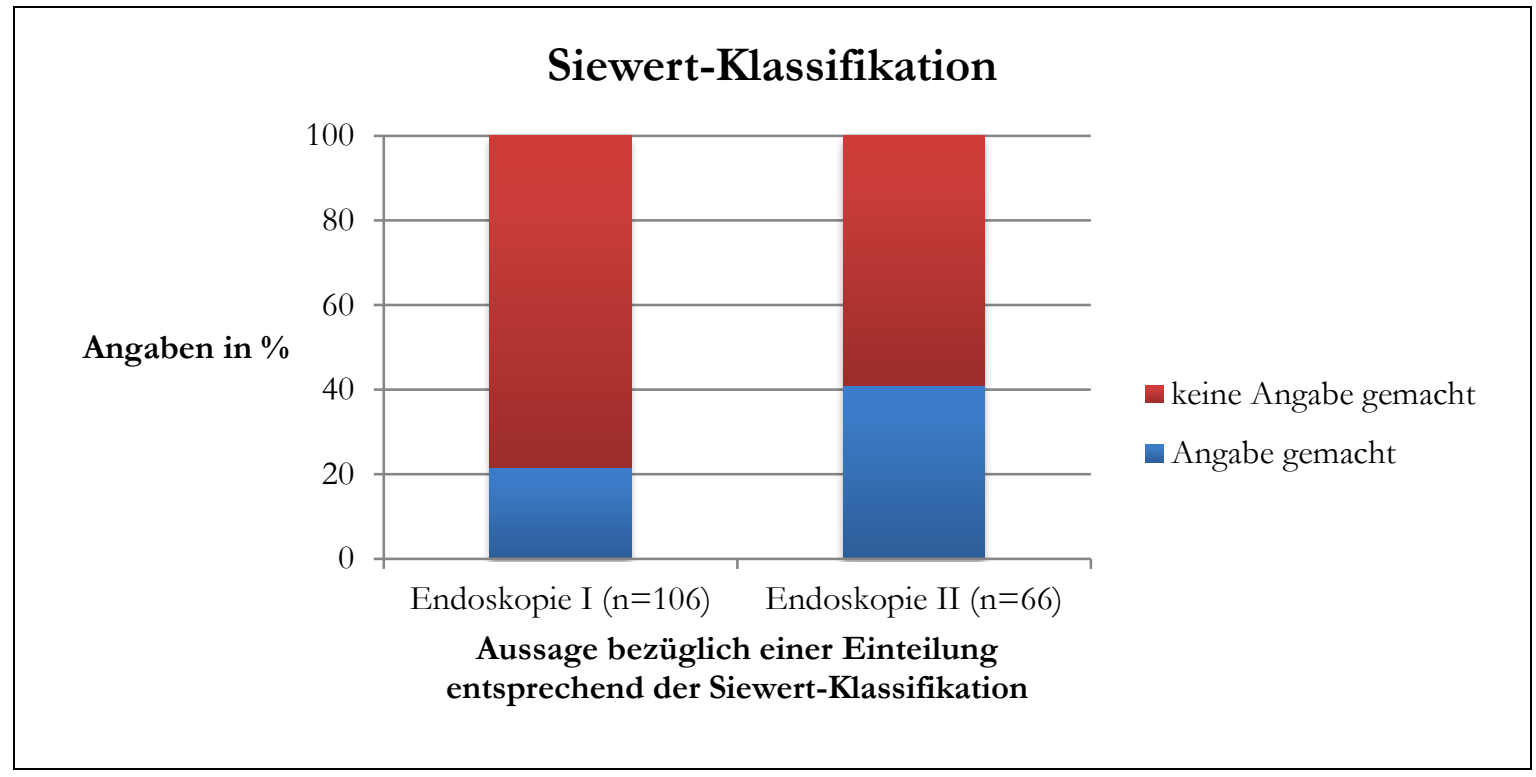

Abbildung 18: Verteilung der angegebenen Siewert-Klassifikationen (AEG-Typ-I, -II und -III) nach Ösophagogastroskopie I und II.

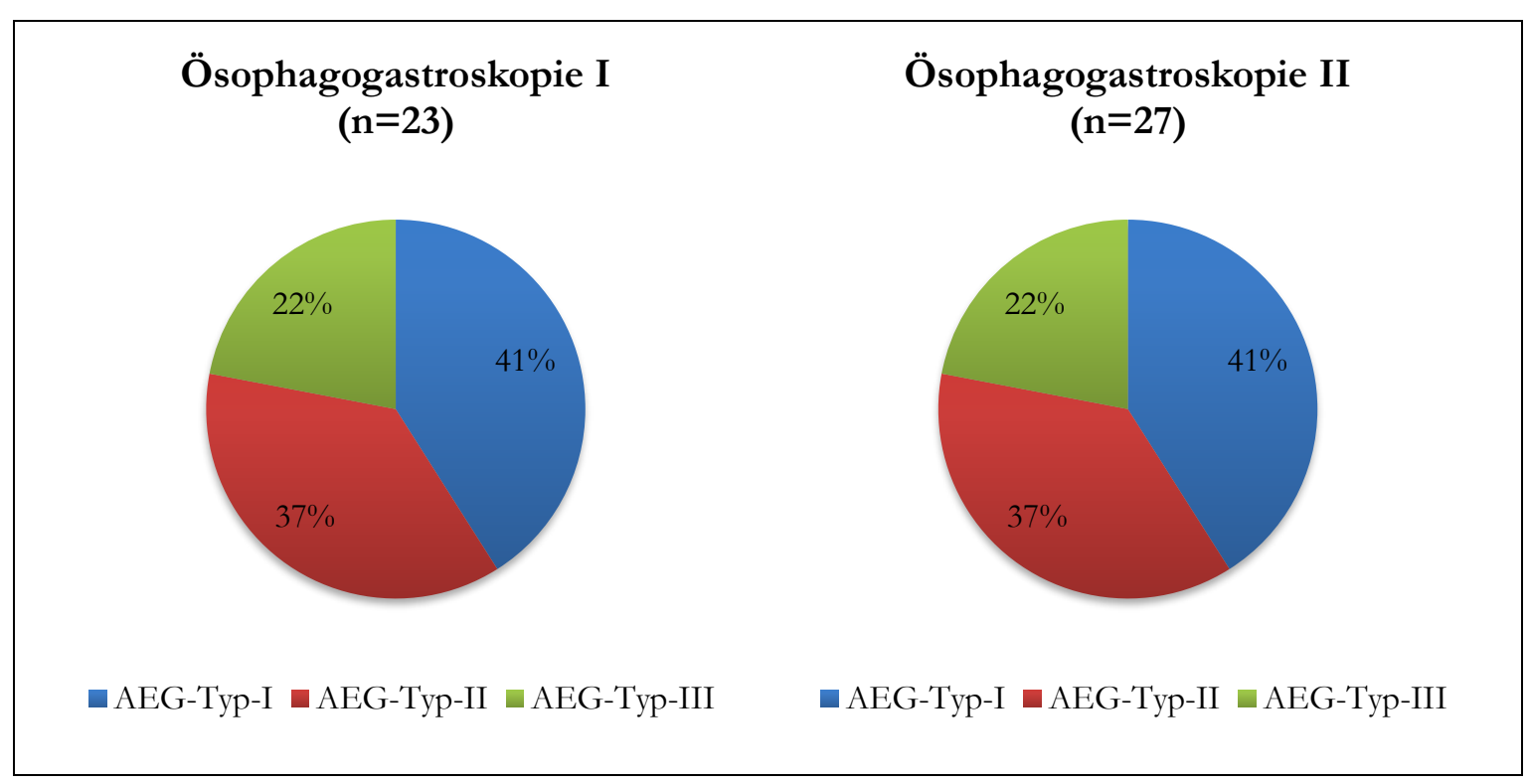


Die genauere Analyse zeigt, dass bei vier Patienten die im Rahmen einer ersten Ösophagogastroskopie vorgenommene Einteilung anhand der Siewert-Klassifikation durch die Befunde der zweiten Untersuchung bestätigt wurde. Bei den anderen 19 Patienten, bei denen in den Befunden einer primären Endoskopie eine entsprechende Einteilung vorlag, kam es nicht zu einer Wiederholung dieser diagnostischen Maßnahme. Somit liegt nach erster und zweiter Endoskopie bei insgesamt 46 Patienten eine Siewert-Klassifikation vor.

\subsubsection{Vergleich der Angaben von den an der Universitätsmedizin Göttingen durchgeführten Ösophagogastroskopien}

Um zu vergleichen ob Unterschiede hinsichtlich der Häufigkeit gemachter Angaben zum Beispiel bezüglich der Z-Linie, dem Beginn des Tumors oder der Passierbarkeit des Tumors nach erster bzw. zweiter Ösophagogastroskopie bestehen, wurden die Dokumente derjenigen Patienten ausgewertet, bei denen die Endoskopien an der Universitätsmedizin Göttingen durchgeführt wurden. Eine erste Ösophagogastroskopie fand bei 35 Patienten an der UMG statt, eine Folgeuntersuchung bei 73 Patienten. Die Analyse der Befundberichte hat die in Tabelle 2 aufgeführten Daten ergeben. Nach der ersten Endoskopie lag eine Zentimeterangabe zur Lage der Z-Linie in 14\% der Fälle vor. Eine Aussage zum möglichen Vorhandensein eines Barrett-Ösophagus wurde bei 63\% der Patienten getroffen. Bei 6\% der Patienten kam es zur Diagnose eines Barrett-Ösophagus, bei 57\% der Patienten wurde das Vorhandensein verneint und in 37\% der Fälle wurden keine Angaben dokumentiert. Beginn und Ende des Tumors wurden in 23\% der Fälle als Zentimeterangabe angegeben, bei 9\% der Patienten gab es lediglich eine Zentimeterangabe zum Lokalisationsbeginn des Tumors, bei 6\% der Patienten war der Tumor nicht passierbar und es wurde daher nur der Beginn der Neoplasie als Zentimeterangabe beschrieben. Eine Lokalisationsangabe des Tumors im Freitext fand sich in $80 \%$ der Befundberichte. Die Siewert-Klassifikation wurde in 12\% der Fälle dokumentiert. In $80 \%$ der Befunde ließ sich eine Angabe zum mutmaßlichen Tumorursprung finden (bei 17\% dieser Patienten lag der Tumor im Ösophagus, bei 20\% der Patienten am gastroösophagealen Übergang und in 43\% der Fälle befand sich die Neoplasie im Magen). Bei der Auswertung der Befunde der zweiten Ösophagogastoskopie zeigte sich, dass die Lage der Z-Linie bei 37\% der Patienten mit einer Zentimeterangabe dokumentiert wurde, bei 63\% der Patienten finden sich Angaben zum möglichen Vorhandensein eines BarrettÖsophagus (bei 7\% dieser Patienten wurde eine Metaplasie nachgewiesen, bei 56\% der Patienten wurde das Vorhandensein verneint). Bei 56\% der Patienten wurden der Beginn und das Ende des Tumors mit einer Zentimeterangabe beschrieben, bei weiteren 14\% kam es lediglich zu einer Dokumentation des Tumorbeginns. Bei 1\% der Patienten war der Tumor nicht passierbar und somit wurde nur der Beginn der Neoplasie mit einer Zentimeterangabe dokumentiert. 56\% der Befundberichte enthielten Angaben zur Tumorlokalisation im Freitext, eine Einteilung nach der Siewert Klassifikation wurde bei 20\% der Patienten vorgenommen. In 81\% der Befunde fanden sich Angaben zum 
mutmaßlichen Ursprung des Tumors, in 34,3\% der Fälle wird dieser im Ösophagus gesehen, bei 21,9\% der Patienten liegt der mutmaßliche Ursprung des Tumors im gastroösophagealen Übergang und bei $24,7 \%$ der Patienten im Magen. Bei den verbleibenden 19,2\% der Patienten finden sich keine Angaben hierzu.

Tabelle 2: Gegenüberstellung der Häufigkeit der dokumentierten Angaben bei den an der UMG durchgeführten Ösophagogastroskopien. Endoskopie I ( $n=35)$, II ( $n=73$ ).

\begin{tabular}{lcc}
\hline Endoskopie & I & II \\
\hline Z-Linie (mit cm-Angabe) & $14 \%$ & $37 \%$ \\
Barrett-Ösophagus diagnostiziert & $63 \%$ & $63 \%$ \\
Beginn und Ende des Tumors in cm & $23 \%$ & $56 \%$ \\
Lokalisationsbeginn des Tumors in cm & $9 \%$ & $14 \%$ \\
$\begin{array}{l}\text { Tumor nicht passierbar } \\
\text { (nur Lokalisationsbeginn des Tumors in cm) }\end{array}$ & $6 \%$ & $1 \%$ \\
Freitext-Angabe zur Tumorlokalisation & $80 \%$ & $56 \%$ \\
Siewert-Klassifikation angegeben & $12 \%$ & $20 \%$ \\
Angabe zum mutmaßlichen Tumorursprung & $80 \%$ & $81 \%$ \\
\hline
\end{tabular}

\subsection{Endosonographische TN-Klassifikation}

Eine Beschreibung der Eindringtiefe des Primärtumors sowie ein möglicher Befall von regionären Lymphknoten können mittels Endosonographie erfolgen, zur Einteilung dient die TNM-Klassifikation. Eine Endosonographie wurde bei 181 Patienten des Gesamtkollektivs durchgeführt (74\%), eine Wiederholung dieser Untersuchung fand bei 13 dieser Patienten statt (7\%). In einer ersten Endosonographie findet sich bei $90 \%$ der Patienten eine Einteilung für den T-Status, eine Zuordnung zu dem vorliegenden N-Status wird in 83\% der Fälle vorgenommen. In der zweiten Endosonographie wird eine Zuordnung entsprechend dem T-Status bei 69\% der Patienten vorgenommen, der N-Status ist bei $46 \%$ dokumentiert.

Tabelle 3: Verteilung des klinischen T-und N-Stadiums nach Endosonographie I und II.

\begin{tabular}{lcccc}
\hline Parameter & Endosonographie & I & Endosonographie & II \\
\hline & $\mathrm{n}=181$ & in $\%$ & $\mathrm{n}=13$ & in $\%$ \\
\hline $\mathbf{u T}$ & & & & \\
\hline T1 & 21 & 11,6 & 1 & 7,7 \\
T2 & 38 & 21 & 5 & 38,4 \\
T3 & 94 & 51,9 & 3 & 23,1 \\
T4 & 9 & 5 & 0 & 0 \\
T nicht bekannt & 19 & 10,5 & 4 & 30,8 \\
\hline T gesamt & 181 & 100 & 13 & 100 \\
\hline $\mathbf{u N}$ & & & & 2 \\
\hline N positiv & 34 & 18,8 & & 15,4
\end{tabular}




\begin{tabular}{lcccc} 
N negativ & 65 & 35,9 & 3 & 23,1 \\
N1 & 47 & 26 & 1 & 7,7 \\
N2 & 4 & 2,2 & 0 & 0 \\
N nicht bekannt & 31 & 17,1 & 7 & 53,8 \\
\hline N gesamt & 181 & 100 & 13 & 100 \\
\hline
\end{tabular}

In Tabelle 4 ist dargestellt, ob es in den Befunden derer Patienten, bei denen zwei Endosonographien durchgeführt wurden zu einer Übereinstimmung der Zuordnung entsprechend der TNM-Klassifikation gekommen ist, oder ob in der Wiederholung der Untersuchung eine Abweichung bezogen auf diese Klassifikation vorlag.

Tabelle 4: Gegenüberstellung der Einteilung entsprechend der TNM-Klassifikation von den Patienten, bei denen Endosonographie I und II durchgeführt wurden ( $n=13)$.

\begin{tabular}{lcc}
\hline Befundergebnisse & T-Status & N-Status \\
\hline gleiche Klassifikation in Endosonographie I und II & 23,08 & 23,08 \\
höhere Klassifikation in Endosonographie II & 0 & 7,69 \\
niedrigere Klassifikation in Endosonographie II & 38,46 & 7,69 \\
Teilangaben fehlen & 38,46 & 61,54 \\
\hline
\end{tabular}

\subsection{Neoadjuvante Chemo- und Strahlentherapie}

Ergänzend zu der operativen Behandlung eines Ösophagus- oder Magenkarzinoms ist es in einigen Fällen indiziert eine neoadjuvante Chemotherapie oder Radiochemotherpie durchzuführen. Aus den Patientenakten wurden Daten über die Durchführung dieser möglichen Behandlung gesammelt, sowie über eventuell vorzeitig erfolgte Abbrüche und deren Gründe. Vom gesamten Kollektiv wurde bei 58,9\% der Patienten eine neoadjuvante Therapie durchgeführt. Bei 58,6\% dieser neoadjuvanten Behandlungen handelt es sich um eine Chemotherapie und bei 41,4\% um eine Radiochemotherapie. Bei 41,1\% der Patienten des Gesamtkollektivs wurde keine neoadjuvante Therapie durchgeführt. In den folgenden Abbildungen sind die Kollektive dargestellt, bei denen neoadjuvant keine Therapie stattgefunden hat bzw. bei denen es zur Durchführung einer neoadjuvanten Chemotherapie oder Radiochemotherapie gekommen ist. Es ist je für die Patienten mit einem Ösophagus- bzw. Magenkarzinom graphisch dargestellt, wie sich das Kollektiv entsprechend einer Stadiengruppierung nach UICC-Kriterien aufteilt. In der Gruppe der Patienten mit einem Ösophaguskarzinom kam es bei 3,6\% der Patienten aufgrund erhöhter Toxizität zu einer Beendigung der neoadjuvanten Radiochemotherapie. Bei 9,6\% der Patienten, die aufgrund eines Magenkarzinoms eine Chemotherapie erhalten haben, wurde 
ein vorzeitiger Abbruch dieser Behandlung dokumentiert. Zu diesem kam es zum Beispiel in Folge einer allergischen Reaktion oder durch das Vorliegen einer palliativen Situation.

Abbildung 19: Verteilung der Stadiengruppierungen nach UICC-Kriterien bei Patienten mit einem Ösophaguskarzinom, die neoadjuvant keine Therapie erhalten haben $(n=35)$.

\section{keine neoadjuvante Therapie}

$(n=35)$

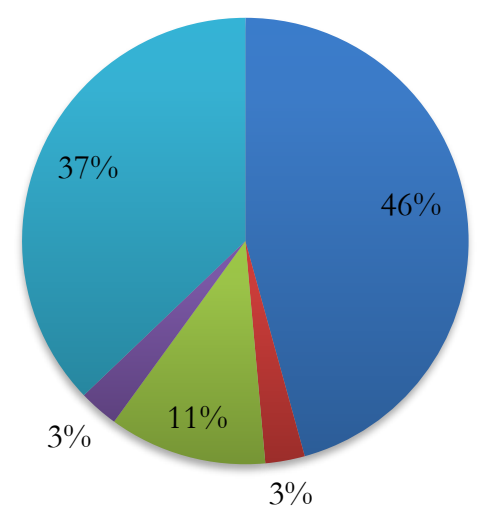

- UICC 1

- UICC 2

- UICC 3

- UICC 4

nicht bekannt

Abbildung 20: Verteilung der Stadiengruppierungen nach UICC-Kriterien bei Patienten mit einem Ösophaguskarzinom, die neoadjuvant eine Chemotherapie erhalten haben $(n=12)$.

\section{neoadjuvante Chemotherapie}

$$
(n=12)
$$

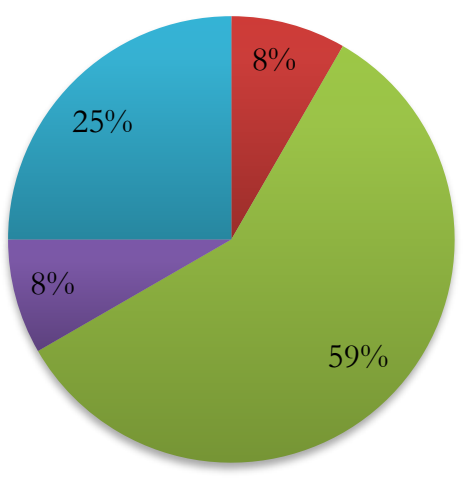

- UICC 2

- UICC 3

UICC 4

nicht bekannt 
Abbildung 21: Verteilung der Stadiengruppierungen nach UICC-Kriterien bei Patienten mit einem Ösophaguskarzinom, die neoadjuvant eine Radiochemotherapie erhalten haben $(\mathrm{n}=56)$.

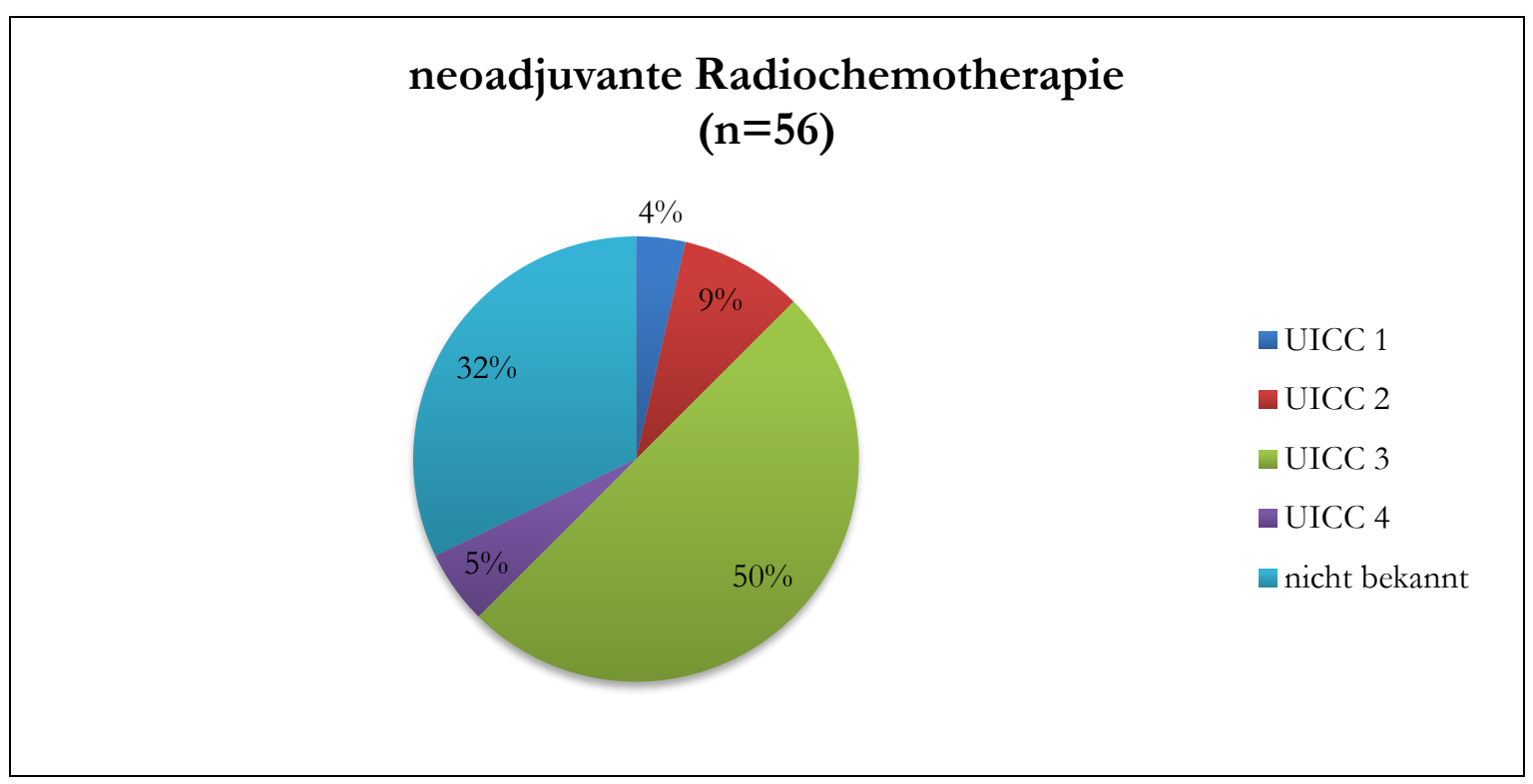

Abbildung 22: Verteilung der UICC-Stadiengruppierungen bei Patienten mit einem Magenkarzinom, die neoadjuvant keine Therapie erhalten haben $(\mathrm{n}=66)$.

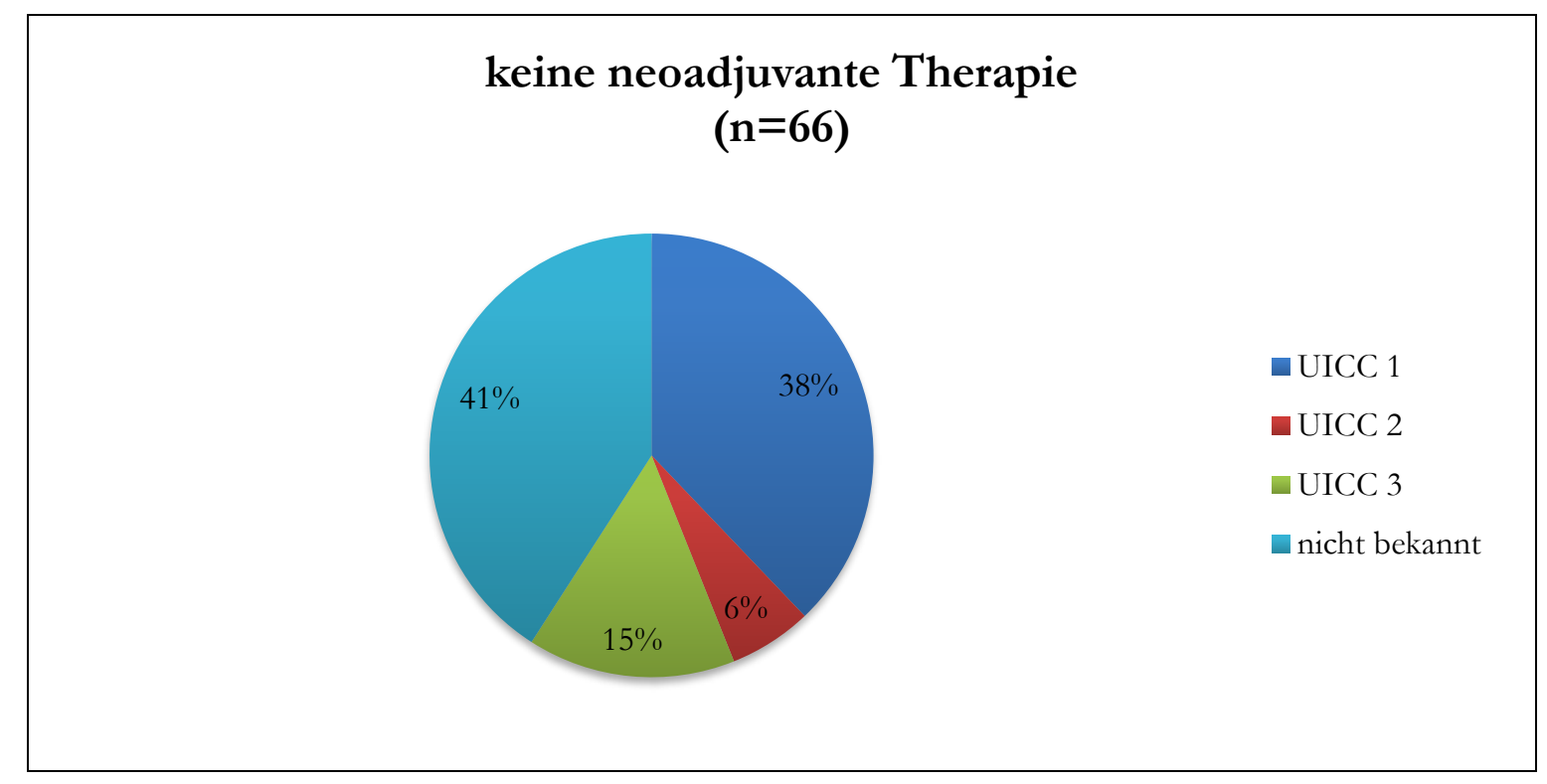


Abbildung 23: Verteilung der UICC-Stadiengruppierungen bei Patienten mit einem Magenkarzinom, die neoadjuvant eine Chemotherapie erhalten haben $(n=73)$.

\section{neoadjuvante Chemotherapie}

$(n=73)$

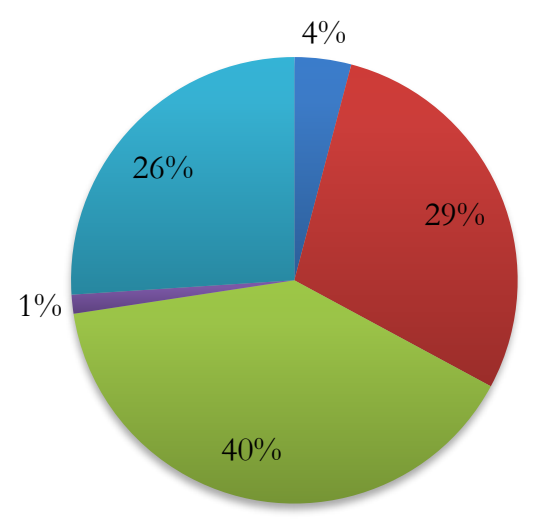

- UICC 1

- UICC 2

- UICC 3

- UICC 4

nicht bekannt

Abbildung 24: Verteilung der UICC-Stadiengruppierungen bei Patienten mit einem Magenkarzinom, die neoadjuvant eine Radiochemotherapie erhalten haben ( $\mathrm{n}=4)$.

\section{neoadjuvante Radiochemotherapie \\ $(n=4)$}

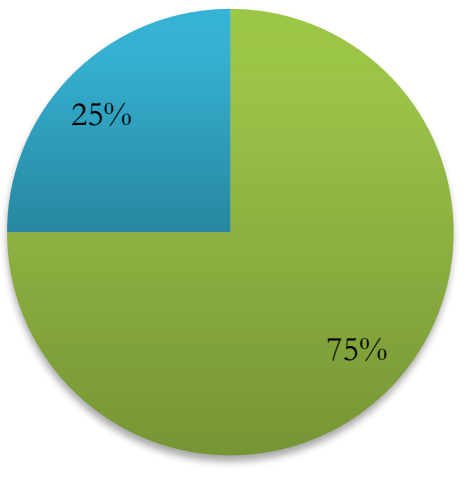

- UICC 3

nicht bekannt

\subsection{Operation}

\subsubsection{Durchgeführte Operationsverfahren}

Insgesamt 240 Patienten des Gesamtkollektivs wurden in der Abteilung für Allgemein- und Viszeralchirurgie der Universitätsmedizin Göttingen operiert. Dies entspricht einem Anteil 
von 97,6\%. Bei vier Patienten kam es aufgrund einer palliativen Situation nicht zur Durchführung einer Operation (1,6\%). Bei weiteren zwei Patienten befanden sich in den Akten keine Informationen bezüglich einer Operation (0,8\%). Die Altersverteilung zum Zeitpunkt der Operation reichte von 22 bis 86 Jahren mit einem durchschnittlichen Alter von 64,5 Lebensjahren. Eine Vielzahl von Operationsverfahren wurde angewendet. Mit 44,2\% am häufigsten durchgeführt wurde bei 106 Patienten die thorako-abdominale Ösophagusresektion mit Magenschlauch. In 70 Fällen (29,2\%) wurde eine Gastrektomie mit D II Lymphadenktomie durchgeführt, bei 47 Patienten (19,6\%) erfolgte eine erweiterte Gastrektomie. Des Weiteren kamen sechs andere Operationstechniken zum Einsatz. Hier wurde unter nicht onkologischen Gesichtspunkten eine Operation durchgeführt, die im Rahmen eines palliativen Konzeptes zur Anwendung kam.

Tabelle 5: Verteilung der beim Gesamtkollektiv durchgeführten Operationsverfahren $(\mathrm{n}=240)$.

\begin{tabular}{lcc}
\hline Operationsverfahren & Patientenzahl & $\mathbf{\%}$ \\
\hline Ösophagusresektion, thorako-abdominal mit Magenschlauch & 106 & 44,2 \\
Ösophagusresektion, partiell mit Magenteilresektion und & 6 & 2,5 \\
Dünndarminterponat & 70 & 29,2 \\
Gastrektomie mit D II - LAE & 1 & 0,4 \\
Gastrektomie mit D I - LAE & 47 & 19,6 \\
erweiterte Gastrektomie & 2 & 0,8 \\
Restgastrektomie & 1 & 0,4 \\
atypische Magenresektion & 1 & 0,4 \\
Billroth I & 5 & 2,1 \\
Billroth II & 1 & 0,4 \\
keine Angaben & &
\end{tabular}

\subsubsection{ICD-Codes C15.5 und C16.0}

Anhand der ICD-Klassifikation wurden die Daten der Patienten analysiert, bei denen die Diagnose C15.5 - Bösartige Neubildung des Ösophagus, unteres Drittel vorlag oder die Diagnose C16.0 - Bösartige Neubildung des Magens, Kardia (Graubner et al. 2016). Bei 63 Patienten liegt die ICD-Klassifikation C15.5 vor und bei 55 Patienten die ICDKlassifikation C16.0. Somit handelt es sich bei dieser Gruppe um 118 Patienten des Gesamtkollektivs. Bei der Auswertung wurde deutlich, dass es für Adenokarzinome des distalen Ösophagus eine Überschneidung in der ICD-Klassifikation gibt. Während formal die AEG-I-Tumoren dem Magen zugeordnet werden, erfolgt klinisch deren Betrachtung als Ösophaguskarzinom. Bei 31,4\% der Patienten wurde in den Endoskopiebefunden eine entsprechende Einteilung vorgenommen. 
Abbildung 25: Verteilung der in den Endoskopiebefunden dokumentierten Angaben bezüglich einer Einteilung anhand der Siewert-Klassifikation bei Patienten mit der Diagnose ICD-C15.5 oder ICD-C16.0 ( $\mathrm{n}=118)$.

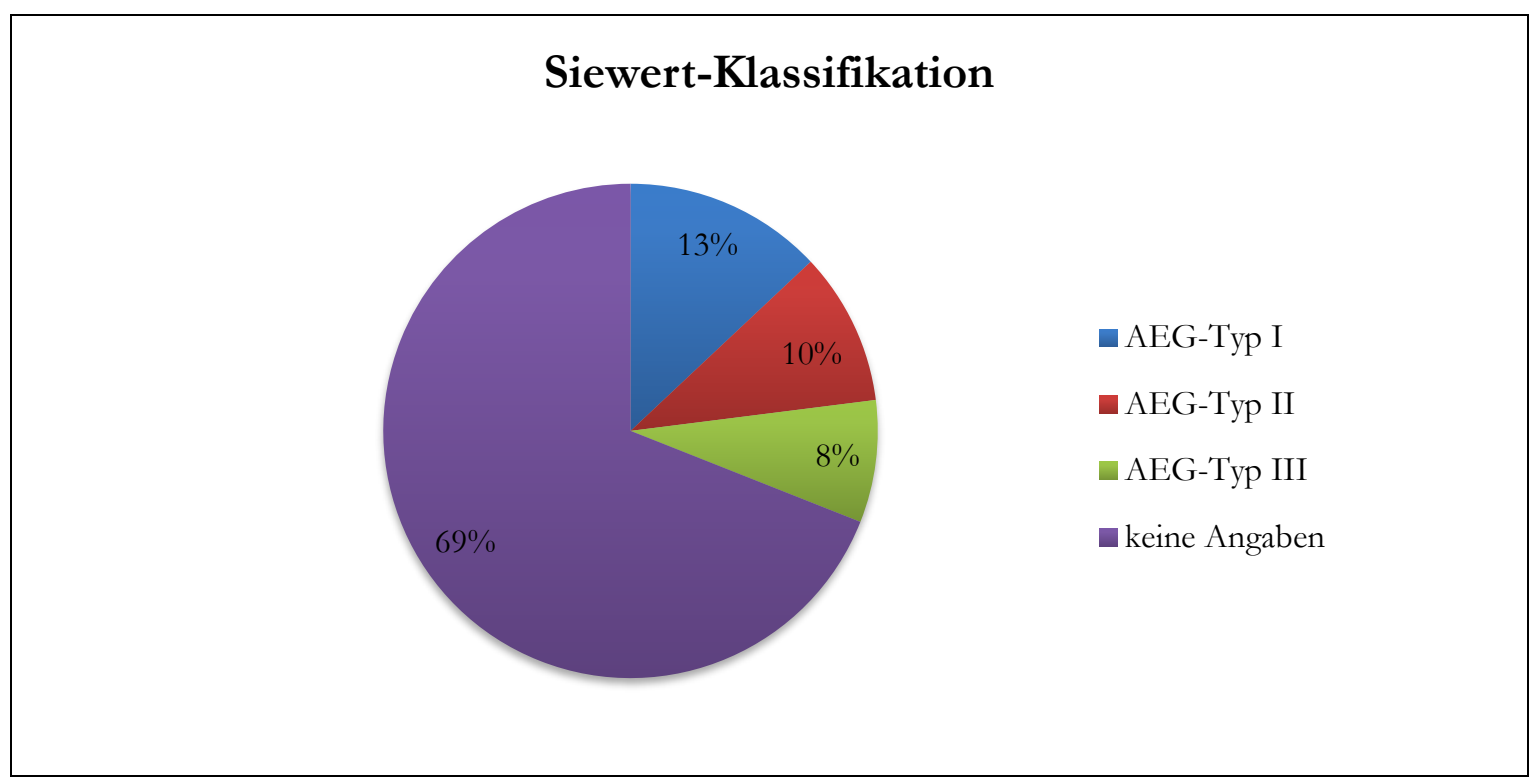

Für dieses Patientenkollektiv wurde außerdem erfasst, ob eine neoadjuvante und/oder eine adjuvante Behandlung erfolgt sind. Bei insgesamt 74,6\% der Patienten kam es zu einer neoadjuvant und / oder adjuvanten Therapie, bei 25,4\% des Kollektivs erfolgte diese nicht.

Tabelle 6: Verteilung der neoadjuvant und/oder adjuvant durchgeführten Therapien bei Patienten mit der Diagnose ICD-C15.5 oder ICD-C16.0.

\begin{tabular}{lcc}
\hline neoadjuvante und/oder adjuvante Therapie & C15.5 (n=63) & C16.0 (n=55) \\
\hline & & \\
neoadjuvante Chemo, adjuvante Chemo & 4,76 & 29,09 \\
neoadjuvante Chemo, adjuvante Bestrahlung & 1,59 & 0 \\
neoadjuvante Chemo, adjuvant keine Therapie & 6,35 & 36,36 \\
neoadjuvant keine Therapie, adjuvant Chemo & 1,59 & 5,45 \\
& & \\
neoadjuvant Radiochemo, adjuvant Radiochemo & 1,59 & 0 \\
neoadjuvant Radiochemo, adjuvant Chemo & 3,17 & 0 \\
neoadjuvant Radiochemo, keine adjuvante Therapie & 49,21 & 7,27 \\
neoadjuvant keine Therapie, adjuvant Radiochemo & 3,17 & 0 \\
& & \\
keine neoadjuvante und keine adjuvante Therapie & 28,57 & 21,82 \\
\hline
\end{tabular}

In einem weiteren Schritt wurden die bei diesen beiden Patientengruppen durchgeführten Operationsverfahren ermittelt (siehe Tabelle 7). Bei einem Patienten mit der Diagnose 
ICD-C16.0 erfolgte keine Operation, wodurch sich für diese Erhebung eine Gruppengröße von 54 Patienten ergibt.

Tabelle 7: Darstellung der durchgeführten Operationsverfahren bei Patienten mit der Diagnose ICD-C15.5 oder ICD-C16.0.

\begin{tabular}{lcc}
\hline Operationsverfahren & C15.5 $(\mathbf{n}=\mathbf{6 3})$ & C16.0 (n=54) \\
\hline $\begin{array}{l}\text { Ösophagusresektion, thorako-abdominal } \\
\text { mit Magenschlauch }\end{array}$ & 88,89 & 24,07 \\
$\begin{array}{l}\text { Ösophagusresektion, partiell } \\
\text { mit Magenteilresektion und Dünndarminterponat }\end{array}$ & 6,35 & 0 \\
Gastrektomie mit D II - LAE & 1,59 & 25,93 \\
erweiterte Gastrektomie & 3,17 & 50 \\
\hline
\end{tabular}

\subsection{Postoperative Histologie}

Die Befunde aus der pathologischen Abteilung, welche die histologische Beurteilung der entsprechenden Präparate aus der Operation darlegen, nahmen folgende Unterteilung vor: Resektate des Ösophagus wurden dem Plattenzellkarzinom oder dem Adenokarzinom zugeordnet bzw. als undifferenziertes Karzinom beschrieben. Bei Magenresektaten erfolgte teils eine spezifischere Unterteilung, zum Beispiel in ein Adenokarzinom vom intestinalen, vom diffusen, vom tubulären oder vom muzinösen Typ. Auch hier wurden Präparate als undifferenziertes Karzinom klassifiziert (siehe Tabelle 8). In 240 von 246 ausgewerteten Patientenakten waren Dokumente über eine durchgeführte Operation in der Abteilung für Allgemein- und Viszeralchirurgie der Universitätsmedizin Göttingen vorhanden. Bei der Analyse zeigt sich, dass der Differenzierungsgrad bei 200 dieser Patienten dokumentiert wurde (83,3\%). Die größten Gruppen bilden mit 42,9\% die Patienten, bei denen der Tumor als mäßig differenziert beschrieben wird und mit 38,8\% die Patienten, die einen schlecht differenzierten Tumor aufweisen. Bei 16,7\% der Patienten ist der Grad der Differenzierung nicht bekannt (siehe Tabelle 9). Ob der Tumor mikroskopisch und makroskopisch im Gesunden entfernt wurde, kann anhand der R-Klassifikation bestimmt werden. Diese ist bei insgesamt 229 Patienten des Kollektivs aufgeführt (95,4\%). Bei 86,7\% dieser Patienten liegen keine Hinweise auf das Vorliegen eines Residualtumors vor, bei 8,3\% der Patienten wird durch die Pathologie die Einteilung R1 vorgenommen, was bedeutet, dass ein mikroskopischer Residualtumor nachweisbar ist. In 0,4\% der Fälle liegt die Beschreibung eines makroskopischen Residualtumors vor. Bei 4,6\% der Patienten findet sich keine Dokumentation bezüglich einer Aussage zum vorliegenden Resektionsstatus (siehe Tabelle 10). 
Tabelle 8: Verteilung der Histologiebefunde der an der Universitätsmedizin Göttingen operierten Patienten ( $\mathrm{n}=240)$.

\begin{tabular}{lcc}
\hline Histologie nach WHO & $\begin{array}{c}\text { Patientenzahl } \\
(\mathbf{n}=\mathbf{2 4 0 )}\end{array}$ & $\begin{array}{c}\text { Angaben } \\
\text { in } \mathbf{0}\end{array}$ \\
\hline Ösophagus (n=97) & 35 & 14,6 \\
\hline Plattenepithelkarzinom & 49 & 20,4 \\
Adenokarzinom & 2 & 0,8 \\
undifferenziertes Karzinom & 11 & 4,6 \\
keine Malignität & & \\
\hline Magen (n=142) & 53 & 22,1 \\
\hline Adenokarzinom & 30 & 12,5 \\
Adenokarzinom (intestinal) & 42 & 17,5 \\
Adenokarzinom (diffus) & 2 & 0,8 \\
Adenokarzinom (tubulär) & 5 & 2,1 \\
Adenokarzinom (muzinös) & 1 & 0,4 \\
undifferenziertes Karzinom & 5 & 2,1 \\
diverse Malignitäten & 4 & 1,7 \\
keine Malignität & & 0,4 \\
& 1 & \\
\hline keine Angaben (n=1) & & \\
\hline
\end{tabular}

Tabelle 9: Verteilung der Angaben zum Differenzierungsgrad $(n=240)$.

\begin{tabular}{lcc}
\hline Differenzierungsgrad & Patientenzahl & Angaben in $\mathbf{0}$ \\
\hline G1 & 2 & 0,8 \\
G2 & 103 & 42,9 \\
G3 & 93 & 38,8 \\
G4 & 2 & 0,8 \\
nicht bekannt & 40 & 16,7 \\
\hline
\end{tabular}

Tabelle 10: Verteilung der Angaben zum Resektionsstatus ( $\mathrm{n}=240)$.

\begin{tabular}{lcc}
\hline Resektionsstatus & Patientenzahl & Angaben in $\%$ \\
\hline R0 & 208 & 86,7 \\
R1 & 20 & 8,3 \\
R2 & 1 & 0,4 \\
nicht bekannt & 11 & 4,6 \\
\hline
\end{tabular}




\subsubsection{Pathologisches TNM-Stadium}

Durch die Pathologie wird postoperativ das TNM-Stadium des Resektats bestimmt. Tabelle 11 listet die Verteilung dieser Einteilung für die Patienten auf, die aufgrund eines Ösophagus- oder Magentumors in der Abteilung für Allgemein- und Viszeralchirurgie der Universitätsmedizin Göttingen operiert worden sind. Entsprechend einer in machen Fällen neoadjuvant durchgeführten Therapie erfolgte die Unterteilung in zwei Gruppen. 53\% der Patienten erhielten vor der Operation eine solche Therapie, bei 47\% der Patienten kam es präoperativ nicht zu einer neoadjuvanten Behandlung. Bei vorbehandelten Patienten wird bei der Dokumentation durch ein dem jeweiligen T-, N- und M-Stadium vorangestelltes „y“ symbolisiert, dass eine neoadjuvante Therapie durchgeführt wurde.

Tabelle 11: Verteilung der pathologischen TNM-Stadien für die Gruppe der neoadjuvant behandelten Patienten sowie für die Patientengruppe, bei der keine neoadjuvante Therapie stattgefunden hat.

\begin{tabular}{|c|c|c|c|c|c|}
\hline \multirow[t]{2}{*}{ Parameter } & \multicolumn{2}{|c|}{ Neoadjuvant behandelt } & \multirow[t]{2}{*}{ Parameter } & \multicolumn{2}{|c|}{ Nicht neoadjuvant behandelt } \\
\hline & Patientenzahl & $\%$ & & Patientenzahl & $\%$ \\
\hline \multicolumn{6}{|l|}{ yp'T } \\
\hline yT0 & 26 & 21,14 & T0 & 2 & 1,83 \\
\hline $\mathrm{y}$ Tis & 0 & 0 & Tis & 1 & 0,92 \\
\hline yT1 & 11 & 8,94 & T1 & 30 & 27,52 \\
\hline yT2 & 24 & 19,51 & $\mathrm{~T} 2$ & 24 & 22,02 \\
\hline y'3 & 54 & 43,9 & $\mathrm{~T} 3$ & 44 & 40,37 \\
\hline y'T4 & 8 & 6,5 & $\mathrm{~T} 4$ & 8 & 7,34 \\
\hline \multicolumn{6}{|l|}{ ypN } \\
\hline $\mathrm{yN} 0$ & 62 & 50,41 & No & 54 & 49,54 \\
\hline $\mathrm{yN} 1$ & 29 & 23,58 & N1 & 24 & 22,02 \\
\hline $\mathrm{yN} 2$ & 20 & 16,26 & $\mathrm{~N} 2$ & 12 & 11,01 \\
\hline yN3 & 12 & 9,76 & N3 & 19 & 17,43 \\
\hline \multicolumn{6}{|l|}{ урМ } \\
\hline $\mathrm{yMx}$ & 118 & 95,93 & $\mathrm{Mx}$ & 103 & 94,5 \\
\hline yM1 & 5 & 4,07 & M1 & 6 & 5,5 \\
\hline
\end{tabular}

Zur besseren Übersicht werden die erhobenen Werte in den folgenden Abbildungen noch einmal graphisch dargestellt. Hierfür wird die Gruppe der neoadjuvant vorbehandelten Patienten der Patientengruppe ohne entsprechende präoperative Therapie direkt bildlich gegenübergestellt. 
Abbildung 26: Graphische Darstellung der Verteilung des pathologischen T-Stadiums der beiden Patientengruppen.

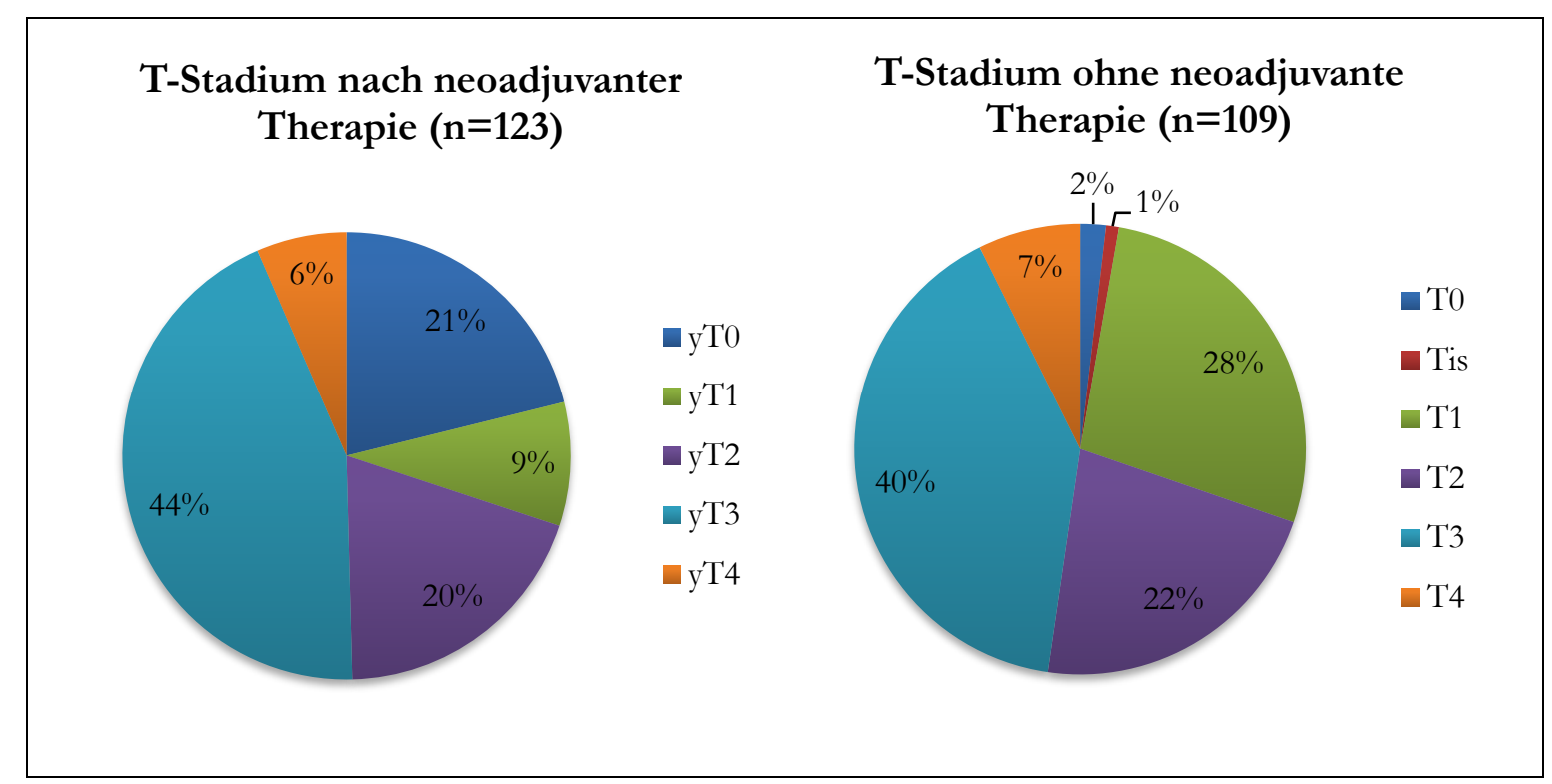

Abbildung 27: Graphische Darstellung der Verteilung des pathologischen N-Stadiums der beiden Patientengruppen.

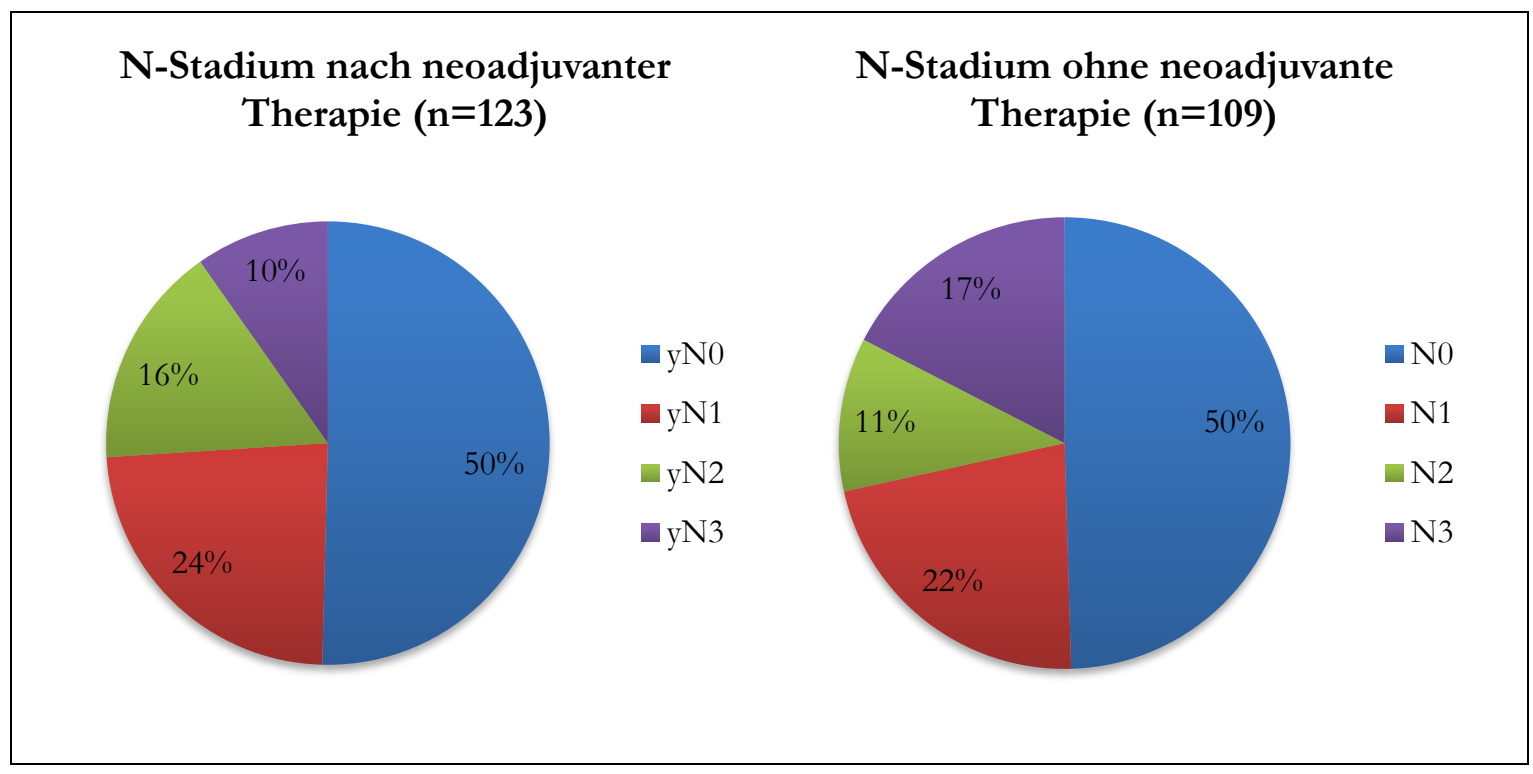


Abbildung 28: Graphische Darstellung der Verteilung des pathologischen M-Stadiums der beiden Patientengruppen.

\section{M-Stadium nach neoadjuvanter Therapie $(n=123)$}

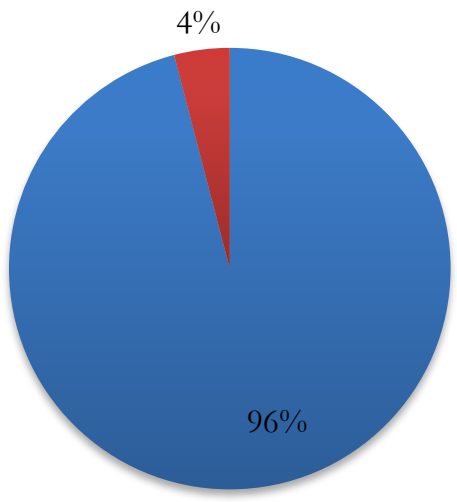

\section{M-Stadium ohne neoadjuvante} Therapie $(\mathrm{n}=109)$

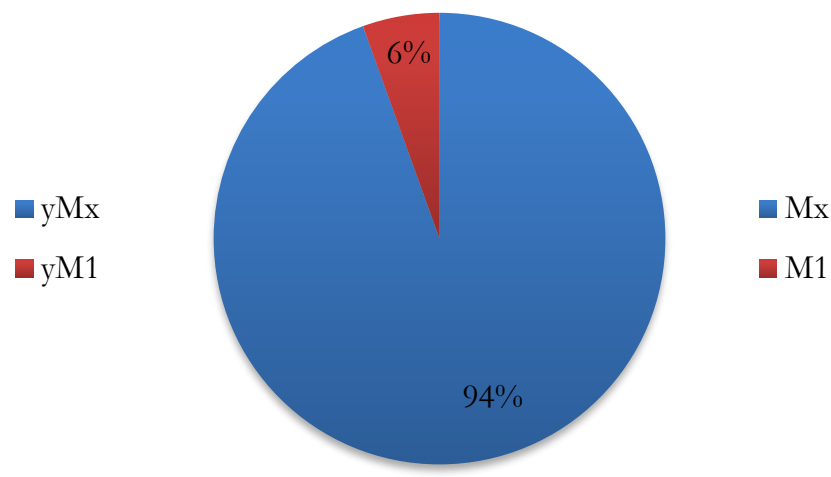

\subsubsection{Lymphknotenstatus}

Durch die Pathologie wurde anhand der einzelnen Resektionspräparate bestimmt, wie viele Lymphknoten in den Operationen jeweils entfernt und untersucht wurden. In 95\% der Fälle konnte mithilfe der Befundberichte aus der Pathologie nachvollzogen werden, wie viele Lymphknoten reseziert wurden. Es sind zwischen einem und 79 Lymphknoten untersucht worden. Der Median im Gesamtkollektiv lag bei 21 untersuchten Lymphknoten. Die Daten wurden in einem weiteren Schritt getrennt analysiert, für Patienten, die aufgrund eines Ösophaguskarzinoms operiert wurden und für die Patientengruppe, die eine Operation aufgrund eines Magenkarzinoms erhielten (siehe Abbildung 21). Es zeigt sich, dass in der Gruppe der Ösophaguskarzinompatienten der Median bei 15 untersuchten Lymphknoten liegt, im Kollektiv der Patienten mit einem Magenkarzinom liegt der Median bei 25 untersuchten Lymphknoten. 
Abbildung 29: Grafik zur Verteilung der Anzahl der untersuchten Lymphknoten bei den Patienten, die aufgrund eines Ösophaguskarzinoms ( $n=99)$ oder eines Magenkarzinoms ( $\mathrm{n}=141)$ operiert wurden.

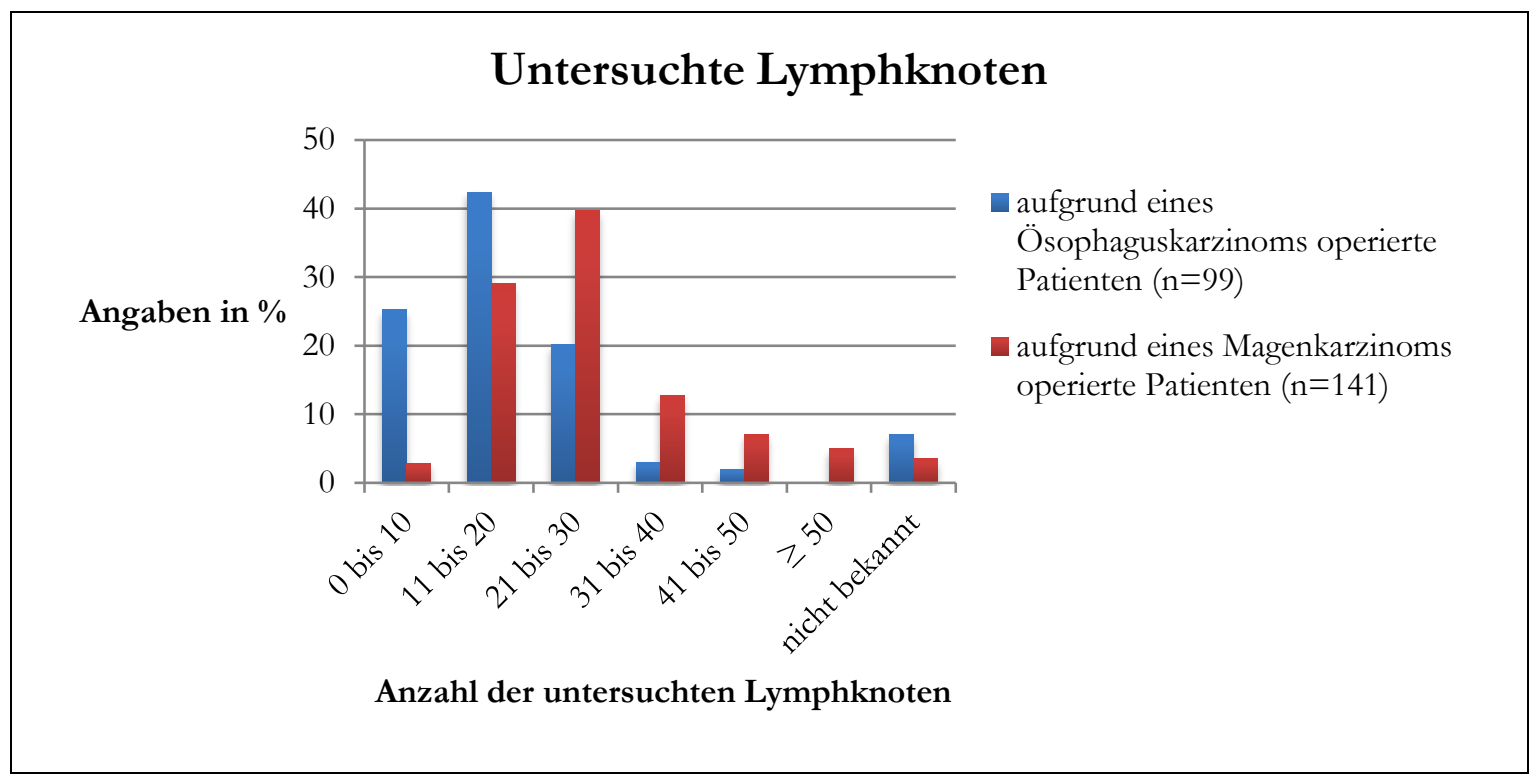

Die Anzahl der befallenen Lymphknoten lag im Gesamtkollektiv zwischen null und 38 mit einem Median von eins. In der Untergruppe der Patienten, die aufgrund eines Ösophaguskarzinoms operiert wurden, wurden null bis neun befallene Lymphknoten reseziert, der Median lag bei Null. Für die Patienten, bei denen eine Operation aufgrund eines Magenkarzinoms durchgeführt wurde lag der Median bei einem befallenen Lymphknoten. Es wurden null bis 38 befallene Lymphknoten reseziert. In insgesamt 114 der 240 Resektionspräparate zeigten sich keine befallenen Lymphknoten (47,5\%).

Abbildung 30: Grafik zur Verteilung der Anzahl der befallenen Lymphknoten bei den Patienten, die aufgrund eines Ösophaguskarzinoms ( $n=99$ ) oder eines Magenkarzinoms ( $\mathrm{n}=141)$ operiert wurden.

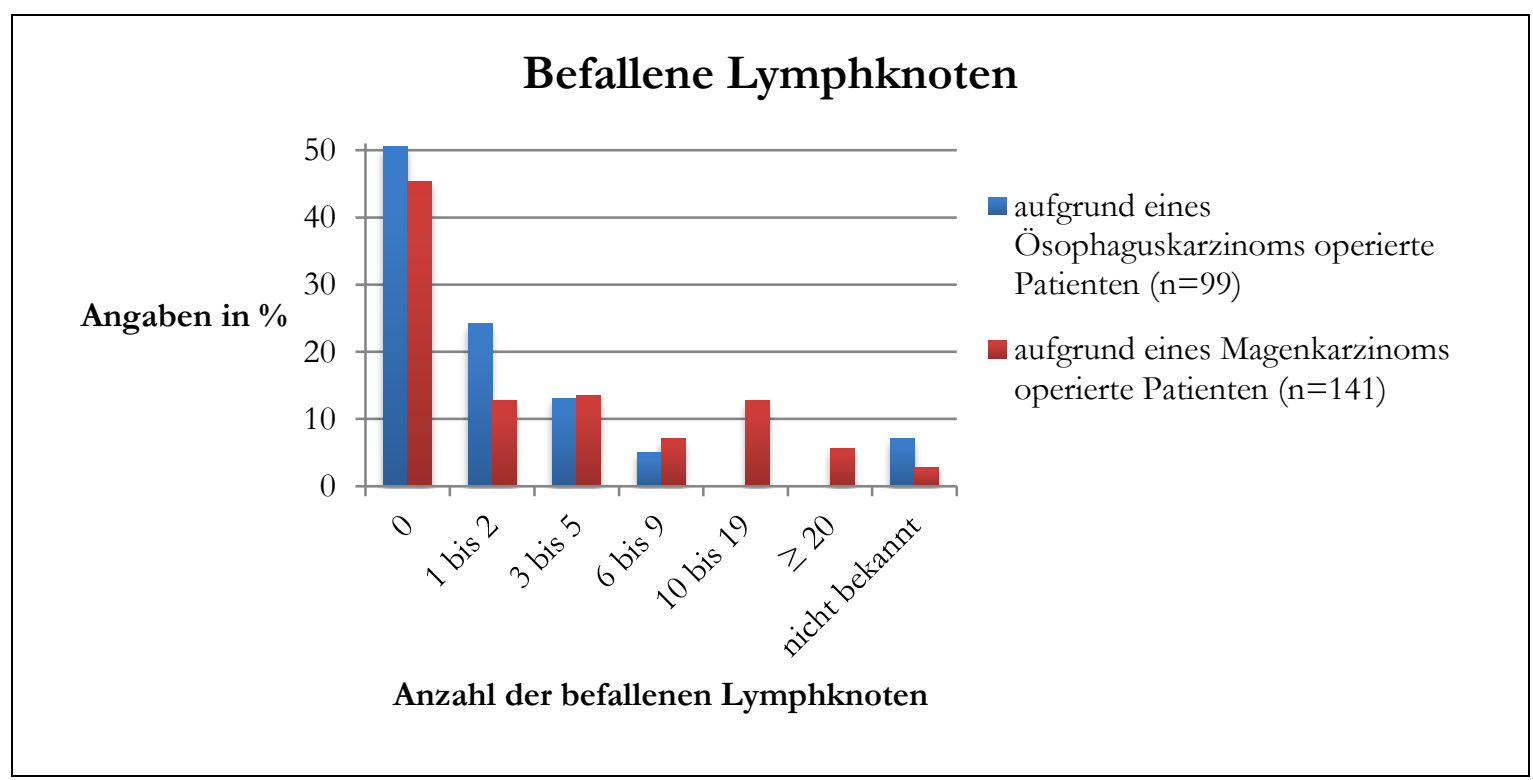


Aus den vorliegenden Werten wurde das Verhältnis von befallenen zu entfernten Lymphknoten berechnet. Es erfolgte eine Einteilung in fünf Gruppen (siehe Abbildung 23). Die Verhältnisse lagen zwischen $0 \%$ befallener zu untersuchter Lymphknoten und 88,5\% befallener zu untersuchter Lymphknoten. Die größten Gruppen bestanden mit 50,5\% bzw. 45,3\% aus den Patienten, bei denen keine befallenen Lymphknoten reseziert wurden und deren Verhältnis somit bei $0 \%$ lag.

Abbildung 31: Grafik zur Verteilung des Verhältnisses von befallenen zu untersuchten Lymphknoten bei den Patienten, die aufgrund eines Ösophaguskarzinoms ( $=99$ ) oder eines Magenkarzinoms ( $\mathrm{n}=141)$ operiert wurden.

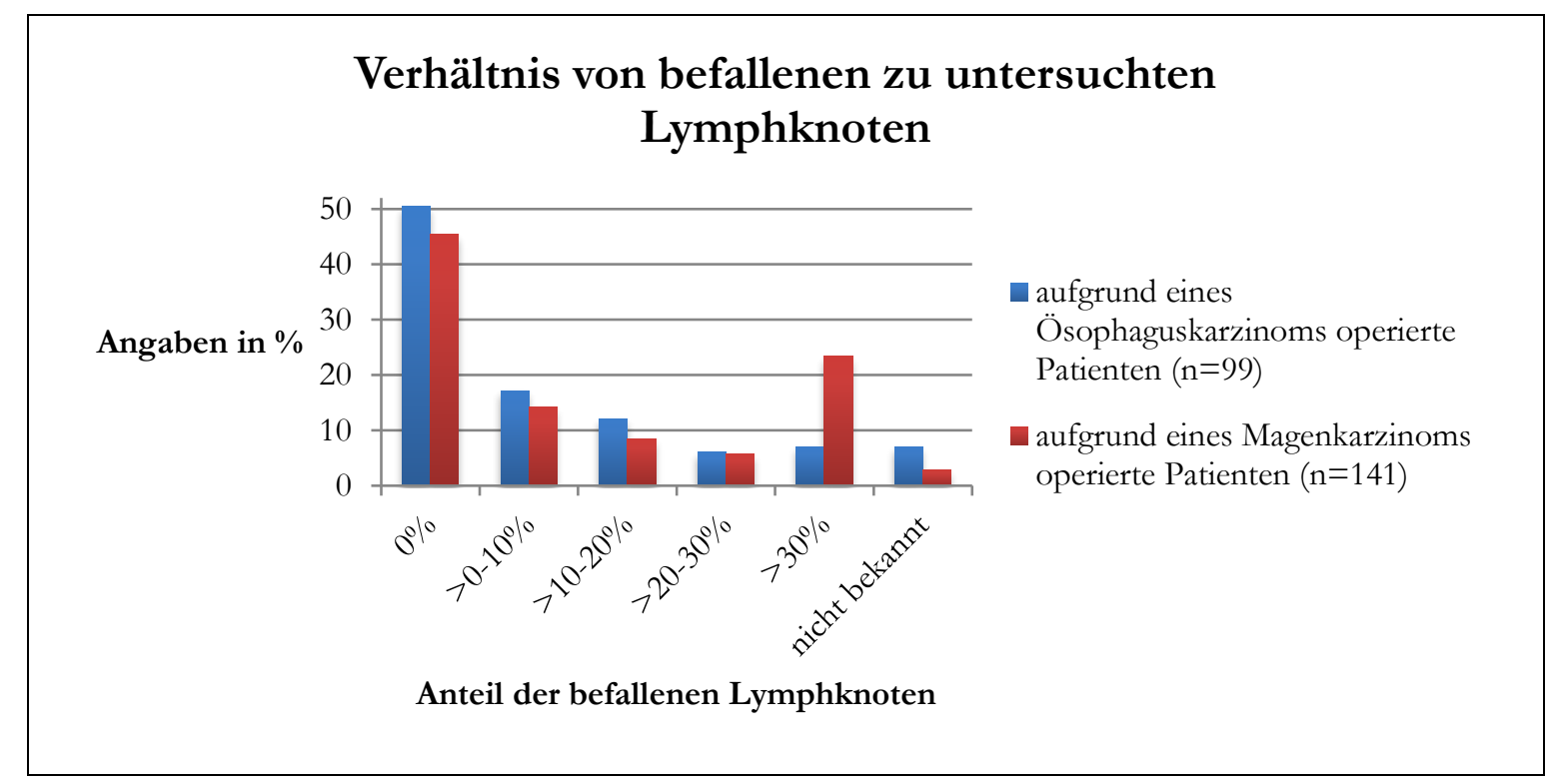

\subsubsection{Barrett-Karzinom - Adenokarzinom auf dem Boden eines Barrett- Ösophagus}

Bei einer Neoplasie des Ösophagus kann es sich um ein Adenokarzinom handeln, welches auf dem Boden eines Barrett-Ösophagus entstanden ist (Faller et al. 2003). Dies wird postoperativ durch die Befundung der Pathologie diagnostiziert. Die Auswertung der durch die Ösophagogastroskopie gewonnenen Biopsie ermöglicht schon im Diagnoseprozess die Bestimmung ob es sich bei der vorliegenden Neoplasie um ein Barrett-Karzinom handelt. Bei den 95 Patienten bei denen mindestens eine Ösophagogastroskopie an der UMG durchgeführt wurde, zeigt sich, dass bei $15,8 \%$ der Patienten entsprechend dem postoperativen histomorphologischen Befund ein Adenokarzinom des Ösophagus vorlag. Bei 40\% dieser Patienten wurde bereits präoperativ anhand der Befunde der Endoskopie die Diagnose eines Barrett-Ösophagus gestellt. Bei 2,1\% der Patienten, bei denen eine Ösophagogastroskopie an der UMG stattgefunden hat, wurde nach dieser Untersuchung die Diagnose eines Barrett-Karzinoms gestellt. Dies wurde durch die postoperativen Befunde jedoch nicht bestätigt. Bei 7,4\% der Patienten wurde präoperativ die Neoplasie des Ösophagus als Adenokarzinom beschrieben, welches auf der Basis eines Barrett- 
Ösophagus entstanden ist, in den postoperativen Unterlagen lag hingegen die Diagnose eines Adenokarzinoms des Magens vor.

Abbildung 32: Graphische Darstellung zu den prä- und postoperativen Aussagen zum Barrett-Karzinom.

\section{Aussagen zum Barrett-Karzinom}

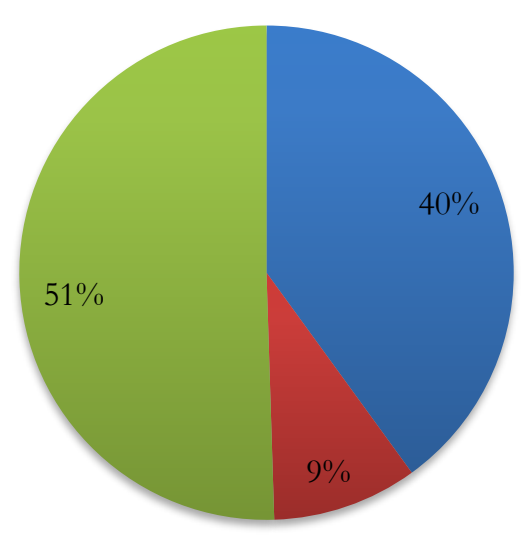

Barrett-Karzinom prä- und postoperativ bestimmt

- präoperativer Barrett-

Karzinombefund, postoperativ nicht bestätigt

kein Barrett-Karzinom beschrieben

\subsection{Adjuvante Chemo- und Strahlentherapie}

Eine adjuvante Therapie wurde bei 72 Patienten des Gesamtkollektivs durchgeführt (29,3\%). $\mathrm{Zu}$ einer adjuvanten Chemotherapie kam es in 86,1\% dieser Fälle, 12,5\% der Patienten wurden mit einer kombinierten Radiochemotherapie behandelt und in 1,4\% der Fälle wurde eine alleinige Bestrahlung durchgeführt. Bei weiteren sieben Patienten (2,9\%) lag die Empfehlung zu einer adjuvanten Behandlung vor, es fehlen jedoch die entsprechenden Daten, ob diese durchgeführt wurde. Ein Patient $(0,4 \%)$ lehnte die weitere Behandlung ab. Eine genaue Aufteilung der adjuvant durchgeführten Behandlungen in den Gruppen der Patienten mit einem Ösophagus- oder Magenkarzinom ist in den folgenden Abbildungen dargestellt. 
Abbildung 33: Darstellung des Einsatzes adjuvanter Therapieoptionen bei Patienten mit einem Ösophaguskarzinom ( $\mathrm{n}=103)$.

\section{Patienten mit einem Ösophaguskarzinom $(n=103)$}

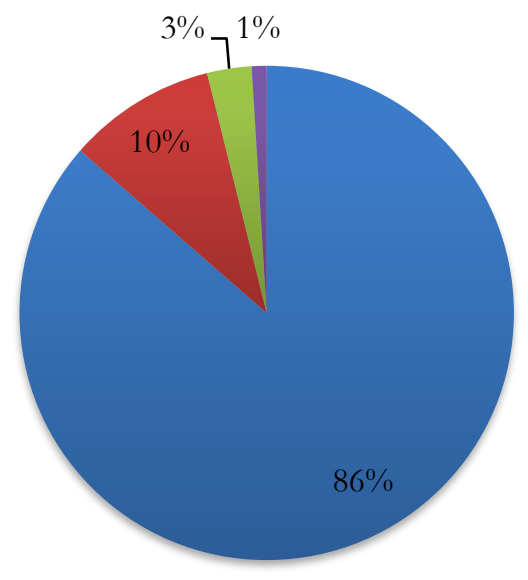

- keine adjuvante Therapie

- adjuvante Chemotherapie

- adjuvante Radiochemotherapie

- adjuvante Radiotherapie

Abbildung 34: Darstellung des Einsatzes adjuvanter Therapieoptionen bei Patienten mit einem Magenkarzinom ( $\mathrm{n}=143)$.

\section{Patienten mit einem Magenkarzinom ( $n=143)$}

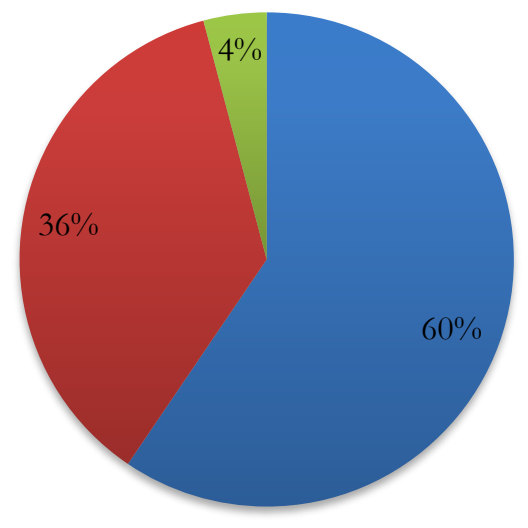

- keine adjuvante Therapie

- adjuvante Chemotherapie

adjuvante Radiochemotherapie

Von den insgesamt 85 Patienten, bei denen neoadjuvant eine Chemotherapie durchgeführt wurde, wurde diese bei 63,5\% der Patienten auch adjuvant durchgeführt. Bei den Patienten, die neoadjuvant eine Radiochemotherapie erhielten, fand adjuvant keine weitere Therapie statt, dies gilt für 60 Patienten. 6,9\% der Patienten des Gesamtkollektivs erhielten nur adjuvant eine Chemotherapie. Bei 11,5\% der Patienten kam es aufgrund von beispielsweise erhöhter Schleimhauttoxizität, prolongierter Übelkeit oder einer Verschlechterung des Allgemeinzustandes zu einem vorzeitigen Abbruch der adjuvanten chemotherapeutischen Behandlung. 


\section{Diskussion}

Ein Ziel dieser Arbeit ist es, den diagnostischen Weg bei den Patienten aufzuzeigen, die aufgrund eines Ösophagus- oder Magenkarzinoms in der Universitätsmedizin Göttingen behandelt wurden. Um diesen Weg zu verdeutlichen, wurden unter anderem in den Befunden der Endoskopie die Aussagen zur Lage der Tumorlokalisation auf ihre Güte und Vollständigkeit hin geprüft. Außerdem wird durch diese Arbeit der aktuell vorliegende Stand der Universitätsmedizin Göttingen dargestellt, was für zukünftige Studien von Bedeutung sein kann.

Die Gesellschaft der epidemiologischen Krebsregister in Deutschland e.V. (GEKID) legt in Zusammenarbeit mit dem Robert Koch-Institut regelmäßig Zahlen vor, die sich auf das Vorkommen von Krebs in Deutschland beziehen. Der letzten Veröffentlichung zufolge wurden für das Jahr 2013 etwa 485.000 Neuerkrankungen an Krebs vorausgesagt. Männer erkranken häufiger an Krebs als Frauen (53,6\% vs. 46,4\%). Das mittlere Erkrankungsalter des in dieser Arbeit untersuchten Patientenkollektivs lag bei knapp 64 Jahren und somit vier Jahre unter dem allgemeinen bundesweiten Durchschnitt an Krebs zu erkranken (RKI 2010b). Während Männer im Schnitt mit 67 Jahren und Frauen mit 71 Jahren an einem Ösophaguskarzinom erkranken, liegt für die Neuerkrankung an einem Magenkarzinom das durchschnittliche Alter bei Männern bei 72 Jahren und bei Frauen bei 75 Jahren. (RKI 2015). Das Alter der Patienten im untersuchten Kollektiv entspricht in etwa den Zahlen der Literatur. Die vorliegenden geringen Abweichungen lassen sich jedoch gut durch die begrenzte Größe der Stichprobe erklären. Betrachtet man die Geschlechterverteilung bei Patienten mit einem Ösophagus- oder Magenkarzinomen zeigt sich, dass laut Angaben im GEKID-Atlas für die Zeit von 2007 - 2012 beim Ösophaguskarzinom ein Anteil von 79\% der Erkrankten auf männliche Patienten fällt und ein Anteil von 21\% auf weibliche Patienten (GEKID-Atlas 2016). In dem für diese Arbeit untersuchten Patientenkollektiv lag der Anteil männlicher Ösophaguskarzinompatienten bei 86\%, der Anteil der weiblichen Patienten lag bei 14\%. Bei der Betrachtung dieser Zahlen in Bezug auf Angaben zum Magenkarzinom, zeigt sich, dass in Deutschland 59\% der Magenkarzinome bei Männern auftreten und 41\% bei Frauen (GEKID-Atlas 2016). Die vorliegende Datenerhebung hat ergeben, dass an der UMG 64\% der Magenkarzinompatienten männlich waren und 36\% weiblich. Anhand dieser Parameter kann das Kollektiv als repräsentativ bezeichnet werden, da es sich mit den Angaben aus der Literatur deckt.

Die Verteilung des Body-Mass-Index des Gesamtkollektivs lässt sich mit der Verteilung, bezogen auf die erwachsene deutsche Bevölkerung, vergleichen, wie in Abbildung 3 und 4 dargestellt. Deutlich wird allerdings, dass die Patienten, die an einem Plattenepithelkarzinom des Ösophagus erkrankt sind, im Durchschnitt einen niedrigeren Body-Mass-Index aufweisen also solche Patienten mit einem Adenokarzinom (siehe 
Abbildung 5). Eine Erklärung dafür liefern vorliegende Daten, welche einen Zusammenhang zwischen Plattenepitehlkarzinom und Alkoholkonsum nachweisen, welcher wiederum mit Untergewicht assoziiert ist (Bollschweiler und Ell 2004). Als Risikofaktoren für die Entstehung eines Plattenepithelkarzinoms des Ösophagus gelten unter anderem der Konsum von Alkohol und Nikotin. Für die Entstehung eines Adenokarzinoms sind ebenfalls Nikotin sowie gastroösophagealer Reflux und das Vorliegen eines Barrett-Ösophagus gesicherte Risikofaktoren (Leitlinie Ösophaguskarzinom 2015). Alkoholkonsum hat in der Kombination mit dem Konsum von Nikotin eine potenzierende Wirkung in Bezug auf ein erhöhtes Ösophaguskarzinomrisiko (Singer und Teyssen 1999). Die Auswertung hat ergeben, dass bei gut 2/3 der Patienten mindestens einer der entscheidenden Risikofaktoren vorliegt. Bei 36\% der Patienten des Gesamtkollektivs liegt ein Nikotinabusus vor, und 15\% der Patienten nehmen regelmäßig Alkohol zu sich. Bei insgesamt 10\% dieser Patienten sind beide Punkte zutreffend. 25\% der Patienten geben an, unter Refluxsymptomatik zu leiden. Das Vorliegen eines BarrettÖsophagus wurde bei 15\% der Patienten durch eine Endoskopie bestätigt, bei insgesamt 85\% des Gesamtkollektivs wurde somit das Vorliegen einer entsprechenden Schleimhautveränderung verneint bzw. wurden diesbezüglich keine Angaben dokumentiert. Vor allem bei Patienten mit einem Karzinom im distalen Ösophagus würde man bei einer größeren Patientenanzahl mit einer Schleimhautveränderung im Sinne einer BarrettMukosa rechnen, da sich Adenokarzinome in diesem Bereich mehrheitlich aufgrund dieser intestinalen Metaplasie entwickeln (Ott et al. 2000). Die Auswertung zeigt, dass nur bei jedem vierten Patienten mit einem distalen Ösophaguskarzinom in den Endoskopiebefunden eine Barrett-Mukosa beschrieben ist. Es ist endoskopisch schwer, eine negative Aussage in Bezug auf diese Fragestellung zu treffen, also klar zu definieren, dass keine Barrett-Mukosa vorliegt. Dies wird durch die große Anzahl der Befundberichte deutlich, in denen eine entsprechende Angabe, die das Vorhandensein einer BarrettMukosa bejaht oder verneint, komplett fehlt. Die Parameter Nikotinabusus, Alkoholkonsum und Refluxsymptomatik wurden anhand von Angaben im Anamnesebogen oder im Anästhesieprotokoll erhoben. Wenn man in Betracht zieht, dass in der westlichen Welt 80\% der Plattenepithelkarzinome mit Nikotin- und Alkoholkonsum in Verbindung gebracht werden (Bareiß et al. 2002), liegen hier relativ niedrige Ergebniswerte vor. Dies könnte darauf zurückzuführen sein, dass gerade die Angaben im Anamnesebogen in vielen Fällen lückenhaft sind, abhängig vom Untersucher und den Umständen der Anamneseerhebung. Auch wird offensichtlich, dass eine standardisierte explizite Nachfrage bezüglich dieser Parameter bzw. Risikofaktoren nicht erfolgt ist. Die Dysphagie gilt als Leitsymptom des Ösophaguskarzinoms. In einer älteren Arbeit wird dieses Symptom bei 87\% der Patienten mit einem Ösophaguskarzinom anamnestisch erhoben (Rosen 1990). In der vorliegenden Arbeit gaben lediglich 51\% der Patienten mit einem Ösophaguskarzinom eine Dysphagie an. Dieser Wert erscheint als zu gering und könnte ebenfalls durch die lückenhafte Anamneseerhebung erklärt werden. Bei der Erhebung der Anamnese ist unter 
anderem entscheidend, wie viel Zeit für diese zur Verfügung steht (Blum und Müller 2003), ob eine klare Kommunikation zwischen Patient und aufnehmendem Personal möglich ist und zudem, wie ausführlich die gewonnenen Informationen dokumentiert werden (Püschmann et al. 2006). Retrospektive Analysen zeigen, dass die Dokumentation ein Problem darstellt. Zukünftig sollte ein Fokus darauf liegen, eine bessere Dokumentation der Anamnese inklusive der Risikofaktoren zu ermöglichen. Die existierenden Anamnesebögen geben dafür eine gute Grundlage, die Auswertung der Patientenunterlagen für diese Arbeit hat allerdings gezeigt, dass trotz bestehender Vorlagen in vielen Fällen nur eine lückenhafte Dokumentation vorliegt.

Die vollständige endoskopische Ösophagogastroskopie stellt das Standardverfahren zur Diagnosestellung von Neoplasien im Bereich des Ösophagus und Magens dar (Leitlinie Magenkarzinom 2012). Ziel dieser Untersuchung sollte sein, dass im Anschluss eine klare Vorstellung bezüglich der Lage des Tumors möglich ist. Dies gilt insbesondere, wenn es sich um eine Neoplasie im Sinne eines Adenokarzinoms im gastroösophagealen Übergang handelt. Zudem sollte vom Endoskopeur Stellung dazu genommen werden, ob bei dem Patienten eine Barrett-Mukosa vorliegt. Auch die Ausdehnung ist von therapeutischer Notwendigkeit. Im Rahmen resezierender Verfahren muss diese als Präkanzerose geltende Veränderung in toto reseziert werden. Die Datenerhebung hat gezeigt, dass qualitative untersucherabhängige Unterschiede bezüglich der Aussagen zu Lage der Neoplasie und Höhenlokalisation vorliegen. Dies liegt unter anderem an der Tatsache, dass die Endoskopie in sehr unterschiedlichen medizinischen Institutionen durchgeführt wurde und es für diese diagnostische Maßnahme keine einheitliche Befunddokumentation gibt. Die Datenerhebung hat ergeben, dass in einem ersten diagnostischen Schritt insgesamt 234 Ösophagogastroskopien zum Staging stattgefunden haben, diese wurden von 117 unterschiedlichen Endoskopeuren in diversen medizinischen Einrichtungen durchgeführt. Diese Vielzahl an Untersuchern macht deutlich, dass es wichtig ist, ein einheitliches System zu etablieren, welches die Minimalanforderungen an die während der Endoskopie gewonnenen Erkenntnisse festlegt, um die gewonnenen Daten zu sichern. Für die individuelle Therapieplanung sind Informationen über die genaue Lage der Neoplasie von enormer Bedeutung. Diese Informationen können sowohl in Form von Zentimeterangaben, die den Beginn und das Ende des Tumors in Zentimetern ab Zahnreihe angeben, sowie in einer im Freitext formulierten Lagebeschreibung vorliegen. Darüber hinaus ist die Lokalisation der Z-Linie. Ohne weitere pathologische Veränderungen zeigt die Z-Linie den Übergang von dem Ösophagus in den Magen an und dient zur Klassifikation von AEG-Tumoren (Siewert et al. 1987). Dies kann jedoch durch das Vorliegen von Refluxveränderungen der Schleimhaut wesentlich verändert sein, sodass die Z-Linie nach oral wandert. Weitere Parameter wie z. B. das Vorliegen einer axialen Hernie sind wichtig, um die exakte Lage des Tumors zu bestimmen. Diese ist für die passende Therapie von essentieller Bedeutung. Die Auswertung der ersten endoskopischen Befunde hat bezüglich dieser Information ergeben, dass sich mit gut 45\% die Mehrzahl der 
Neoplasien im gastroösophagealen Übergang befanden. Gut 23\% der Tumoren lagen demnach im Ösophagus und gut 31\% im Magen. Zur genaueren Analyse wurde das Patientenkollektiv weiter aufgeteilt in eine Gruppe von Patienten, bei denen die Endoskopie an der Universitätsmedizin Göttingen durchgeführt wurde, also im später operierenden Zentrum, und in eine Gruppe von Patienten, bei denen diese Diagnostik außerhalb der UMG stattgefunden hat. Die Auswertung hat ergeben, dass es bei einer Zweituntersuchung zu einer Steigerung bezüglich der Informationsdichte gekommen ist, beispielweise in Bezug auf die Lokalisationsbeschreibung des Tumors, unabhängig von der durchführenden Klinik. Diese Steigerung ist zwar deutlich, stellt allerdings immer noch kein zufriedenstellendes Ergebnis dar. So fanden sich beispielsweise in 63\% der Fälle auch nach einer zweiten Endoskopie keine Angaben bezüglich der Lage der Z-Linie.

In Bezug auf die Angaben zum Vorliegen einer Barrett-Mukosa erzielt die Datenauswertung ebenfalls keine zufriedenstellenden Ergebnisse. In einer ersten Endoskopie, welche als Standarduntersuchung zur Diagnostik einer Barrett-Mukosa eingesetzt wird (Behrens et al. 2011) wurde lediglich bei 60\% der Patienten Stellung diesbezüglich genommen. Das Vorliegen dieser Metaplasie wurde bei knapp 15\% der Patienten beschrieben. In einer zweiten Endoskopie findet sich bei 55\% der Patienten eine Aussage, hier lag eine Barrett-Mukosa bei 11\% der Patienten vor. Größter Risikofaktor für ösophageale Adenokarzinome ist ein bestehender Reflux mit einem als Folgeerscheinung auftretendem Barrett-Ösophagus (Souza und Spechler 2005). Gerade bei dem Kollektiv, bei dem die Neoplasie im distalen Ösophagus vorliegt, wäre somit eine Veränderung im Sinne einer Barrett-Mukosa zu erwarten gewesen. Die Analyse zeigt für dieses Patientenkollektiv allerdings nur in jedem viertel Fall das Vorliegen dieser Metaplasie. In gut $40 \%$ der Fälle liegt in den Befunden keine Aussage diesbezüglich vor. Angaben zur weiteren Klassifizierung der Schleimhautveränderung in eine kurz- oder langstreckige Barrett-Mukosa fanden sich nur in knapp 4\% der Befunde. Insbesondere für Patienten mit einer langstreckigen Läsion besteht für die Entstehung einer Neoplasie des Ösophagus ein erhöhtes Risiko (Pohl et al. 2016). Dies lässt vermuten, dass bei deutlich mehr Patienten eine langstreckige Barrett-Mukosa zum Zeitpunkt der Diagnose vorlag, als in den Befunden dokumentiert wurde. Es ist also anzunehmen, dass in vielen Fällen eine unzureichende Befundung stattgefunden hat und dass bei einer Vielzahl der Patienten der tatsächlich vorliegende Zustand nicht korrekt wiedergegeben wurde. Diese Erkenntnis gibt einen Anreiz, die Qualität der Datenerfassung zukünftiger Untersuchungen zu verbessern.

Bei der Durchführung einer Ösophagogastroskopie sollte im Regelfall bei Vorliegen eines suspekten Befundes die Entnahme einer Biopsie erfolgen (Leitlinie Ösophaguskarzinom 2015). Anhand der dadurch gewonnenen Gewebeprobe kann durch die histologische Auswertung Stellung zu verschiedenen Aspekten genommen werden. So wird beispielsweise eine Einteilung des Befunds nach Definition der WHO vorgenommen, eine Bestimmung des Differenzierungsgrades ist möglich, und bei Magenkarzinomen kann die Laurén-Klassifikation angegeben werden. Die Information über die Laurén-Klassifikation 
ist beispielsweise entscheidend für die weitere Therapieplanung und den zu erzielenden Abstand bei der Resektion (Schmid und Kremer 2002). Die Auswertung der Befunde hat ergeben, dass nur bei jedem dritten Patienten mit einem Magenkarzinom die LauénKlassifikation angegeben war. Diese Erkenntnis sollte Anreiz bieten, zukünftig einen größeren Fokus auf die Erfassung und Dokumentation dieser Information zu legen. Bei der Angabe des Differenzierungsgrads fehlt bei 34\% der Patienten eine Zuordnung. Auch hier wäre es wünschenswert eine lückenlose Dokumentation zu erreichen. Eine histologische Sicherung der Diagnose nach Definition der WHO fand mit gut 95\% bei einem Großteil der Patienten statt. Bei den fehlenden 5\% ist davon auszugehen, dass der Befund in der Akte nicht mehr vorhanden war.

Die Klassifikation nach Siewert ist von steigender Bedeutung, da die Anzahl der gastroösophagealen Übergangstumoren in den letzten Jahren in den westlichen Ländern stark zugenommen hat (Stein et al. 2000). Bei der Betrachtung der Endoskopiebefunde hinsichtlich einer Aussage zum Vorliegen eines AEG-Tumors wird deutlich, dass nur in wenigen Fällen eine eindeutige Zuteilung zu einem der drei Typen vorgenommen wurde. Bei den Patienten, bei denen sich die Neoplasie im gastroösophagealen Übergang befindet wurde im Rahmen der ersten Endoskopie nur bei jedem 5. Patient eine Einteilung nach Siewert dokumentiert und lediglich bei $41 \%$ der Patienten war dies nach der zweiten Untersuchung der Fall. Diese Zahlen sind im Hinblick auf die durchgeführten Operationen allerdings gering. Anhand der histologischen Ergebnisse und der Auswertung der Operationsberichte lassen sich Rückschlüsse darauf ziehen, dass bei deutlich mehr Patienten eine Einteilung hätte vorgenommen werden können. So lag bei 180 Patienten laut histologischer Befundung ein Adenokarzinom vor (in 49 Fällen ein Adenokarzinom des Ösophagus, in 132 Fällen ein Adenokarzinom des Magens). Es wird deutlich, dass bei den meisten Patienten mit einem Tumor im ösophagogastralen Übergang eine klare Zuordnung zu einem der drei Tumorarten fehlt. Für die einzelnen AEG-Typen gibt es je eigene chirurgische Therapiekonzepte (Hölscher et al. 2010). Dies bedeutet, dass der Festlegung des Tumors anhand der Siewert-Klassifikation eine enorme Tragweite zuzusprechen ist, welche dem Endoskopeur bekannt sein sollte. Ein fehlendes Vornehmen der Einteilung mag unter anderem auch daran liegen, dass die mit dieser Entscheidung verbundenen Auswirkungen auf das weitere Therapieregime so bedeutend sind und im Einzelfall zu unsichere Befunde vorliegen oder eine klare Bestimmung des Tumors dem Untersucher nicht möglich ist. Dabei ist eine Einordnung anhand dieser Klassifikation für die Planung des weiteren Therapieregimes notwendig. Mit ihr wird der Versuch unternommen, ein System zur Vergleichbarkeit der Therapieergebnisse zu etablieren (Schiesser und Schneider 2009). Auf welcher Basis bei den entsprechenden Patienten eine bestimmte Operation durchgeführt wurde ist aufgrund der retrospektiven Datenerhebung nicht eindeutig zu beantworten. Eine Therapieentscheidung über das operative Vorgehen wird bei operationsfähigen Patienten mit resektablem Tumor unter anderem anhand von 
Berichten der Endoskopie, Endosonographie, Computertomographie oder auch des Ösophagus-Breischlucks je nach vorliegendem klinischen Stadium entschieden.

In einem weiteren Schritt wurden die Daten der Endoskopien verglichen, die an der Universitätsmedizin Göttingen durchgeführt wurden. Bei einer zweiten Ösophagogastroskopie ist dem Endoskopeur der Befund der vorangegangenen Untersuchung im Regelfall bekannt. Der Datenvergleich zeigt, dass in den meisten Fällen die zweite Untersuchung eine höhere Informationsdichte bietet. Bei einigen Patienten sind allerdings auch die Angaben in den Unterlagen der Wiederholungsuntersuchung lückenhaft. Festzuhalten ist, dass zwar ein deutlich sichtbarer Informationszuwachs bei einer wiederholt durchgeführten Ösophagogastroskopie zu verzeichnen ist, dass aber trotz allem für die Therapieplanung relevante Informationen in vielen Fällen unzureichend dokumentiert werden. Es stellt sich die Frage, was die Minimalanforderungen an eine Ösophagogastroskopie sind und wie gut die momentan durchgeführten Endoskopien tatsächlich in Bezug auf die Informationslieferung sind. Kritisch zu betrachten ist die Tatsache, dass es sich bei dieser Arbeit um eine retrospektive Datenerhebung handelt und zum Teil nur inkomplette Daten in die Auswertung eingegangen sind. Diese Tatsache lässt möglicherweise ein größeres Bias vermuten als vorhanden. Dass die Befundberichte der Untersuchungen, die im Behandlungsverlauf durchgeführt wurden, in ihren Aussagen teilweise diskrepante Aussagen enthalten, wurde bei der Datenerhebung für diese Arbeit allerdings deutlich. Der Befund einer auswärtigen - also nicht an der UMG durchgeführten - Ösophagogastroskopie ist in den meisten Fällen nicht aussagekräftig genug, um eine individuelle Therapieentscheidung zu treffen, und die Planung einer Operation ist anhand dieses Befundes nicht möglich. Somit besteht eine deutliche Tendenz zur Veranlassung einer zweiten Endoskopie. Die Tragweite einer diagnostischen Maßnahme sollte sowohl dem Patienten als auch in besonderem Maße dem Untersucher bewusst sein, da sonst eine Re-Endoskopie vorprogrammiert ist. Hier stellt sich die Frage, ob die Durchführung einer Untersuchung vertretbar ist, von der im Vorfeld bekannt ist, dass sie mit großer Wahrscheinlichkeit wiederholt werden muss. Gerade auch unter Berücksichtigung der Tatsache, dass jeder medizinische Eingriff gewisse Risiken für den Patienten birgt. Bei einer Endoskopie besteht zum einen das Risiko, dass Zwischenfälle bedingt durch die zur Sedierung verwendeten Medikamente auftreten (Wehrmann und Riphaus 2008) und zum anderen besteht die Gefahr einer iatrogenen Perforation des Ösophagus (Agha-Mir-Salim et al. 2000), des Weiteren kann es zu einer Infektionserregerübertragung durch unzureichend gereinigte endoskopische Geräte kommen (Bader et al. 2002) und weitere Komplikationen wie Blutungen sind ebenfalls möglich (Eisen et al. 2002). Ein zu nennender Vorteil einer häufig im Vorfeld ambulant durchgeführten Endoskopie besteht jedoch darin, dass während dieser Untersuchung eine Biopsie erfolgt und die Patienten mit pathologischen Vorbefunden im weiter behandelnden Krankenhaus vorstellig werden können. Problematisch ist vor allem, wenn Patienten wiederholt auswärts gastroskopiert werden. Es ist für den auswertigen Untersucher schwierig den vorliegenden Befund zu 
beurteilen, wenn dieser den Tumor im Verlauf nicht behandelt. Eine auswärts durchgeführte Endoskopie zur Tumorsicherung ist sinnvoll, bei einer zweiten Untersuchung sollten jedoch alle nötigen Informationen erfasst werden und diese sollte an einem Zentrum durchgeführt werden, welches auch therapeutisch tätig ist, da dort den betreffenden Personen bekannt ist, welche Informationen für die weitere Planung entscheidend sind. Ziel sollte sein die Rate der Re-Endoskopien aus den oben genannten Gründen gering zu halten. Von ebenfalls hoher Relevanz für die Therapieplanung ist das Wissen über die exakte Lage sowie Längsausdehnung des Tumors (Seehofer et al. 2013). Die Auswertung der Daten hat für diese Angaben keine zufriedenstellenden Ergebnisse geliefert. Beginn und Ende des Tumors wurden in einer ersten Untersuchung nur bei knapp einem Viertel der Patienten dokumentiert, bei einer Folgeendoskopie erfolgte diese Angabe immerhin bei gut der Hälfte der Patienten, was allerdings bedeutet, dass bei fast jedem Zweiten diese Informationen vor der Operation nicht vorliegen. Zu erwähnen ist an dieser Stelle, dass die vorliegende Arbeit aus einer chirurgischen Klinik kommt, die Endoskopien jedoch durch Internisten durchgeführt wurden. Daher spiegeln die Ergebnisse durchaus die unterschiedlichen Anforderungen, Fragestellungen und Probleme der jeweiligen Fächer wider. Aus chirurgischer Sicht ist die Frage nach der Art der notwendigen Operation entscheidend. Die Situation bei AEG-Typ-I und AEG-Typ-IIITumoren ist dabei eindeutig, vorausgesetzt, die Einteilung wurde korrekt vorgenommen, wofür die unterschiedlichen Parameter Hinweise geben. Bei einem AEG-Typ-II-Tumor stellt sich die entscheidende Frage, ob eine Operation von abdominal mit ausreichend Sicherheitsabstand durchführbar ist. Diese kann aktuell in vielen Fällen anhand der Vorliegenden Befunde präoperativ noch nicht zufriedenstellend beantwortet werden.

Als erstes Zwischenfazit dieser Arbeit ist die zukünftige standardisierte Erfassung der klinischen Befunde sowie der Gastroskopie zu fordern. Hierzu sollten Tumorbeginn und ende, Höhe der Z-Linie, Vorhandensein und Ausdehnung einer Barrett-Mukosa sowie oberer und unterer Schließmuskel vorhanden sein. Dies ist bevorzugt in tabellarischer Form darzustellen, sodass die Werte nicht in einem Fließtext untergehen. Bei AEGTumoren ist eine Typisierung anzugeben.

Bei Ösophagus- und Magenkarzinomen besteht neben der endoskopischen Untersuchung als präoperatives Staging sowie im Rahmen der Therapieplanung eine weitere Möglichkeit in der Durchführung einer Endosonographie (Dittler 2002). Mithilfe dieses Verfahrens kann entsprechend der TNM-Klassifikation die Infiltrationstiefe der Tumoren (TKategorie) sowie eine mögliche Lymphnoteninfiltration (N-Kategorie) beurteilt werden (Bösing et al. 2003). Eine Endosonographie erfolgte bei $74 \%$ der Patienten des Gesamtkollektivs, zu einer Wiederholung der Untersuchung kam es lediglich in 7\% dieser Fälle. Eine Gegenüberstellung der Befunde der Patienten, bei denen zwei Endosonographien durchgeführt wurden, zeigt, dass eine gewisse Variabilität darin besteht, wie die Infiltrationstiefe des Tumors bzw. die Lymphknoteninfiltration klassifiziert wurden. Es wurden sowohl die gleichen ( $\mathrm{T}$ - und N-Status), also auch höhere (N-Status) und 
niedrigere ( $\mathrm{T}$ - und N-Status) Klassifikationen, vorgenommen. Dass Veränderungen in beide Richtungen vorliegen, lässt Rückschlüsse darauf ziehen, dass eine gewisse untersucherabhängige Varianz besteht. Bei $26 \%$ der Patienten wurde keine Endosonographie durchgeführt und somit fehlt bei diesen eine präoperative Einordnung der Infiltrationstiefe des Tumors sowie der Lymphknoteninfiltration anhand den Richtlinien der UICC. Die Frage, die sich bei einer retrospektiven Erhebung stellt, ist die, ob es bei der ermittelten Patientengruppe tatsächlich nicht zur Durchführung einer Endosonographie gekommen ist oder ob die im Archiv vorliegenden Akten zum Zeitpunkt der Datenerfassung nicht vollständig waren. Alternativ ist aus dem Klinikalltag auch denkbar, dass nicht passierbare Tumoren als mindestens T3 gewertet werden. Eine exakte Angabe ist nicht möglich. Dies wird dann als Indikation zur Vortherapie gewertet. Eine Dokumentation sollte dennoch erfolgen. Dies zeigt zum einen deutlich, dass bei retrospektiven Analysen die Erfassung ein Problem darstellt. Zum anderen muss insbesondere in solchen Punkten die retrospektive Analyse nachvollziehbar sein, um auch im Nachgang die Indikation zur jeweiligen Therapie nachzuvollziehen.

Durch den Einsatz neoadjuvanter Therapien als zusätzliche Option neben der Durchführung einer Operation hat sich für Patienten mit einem Ösophaguskarzinom ein Überlebensvorteil ergeben (Sjoquist et al. 2011, van Hagen et al. 2012). Im untersuchten Kollektiv haben 2/3 der Patienten eine neoadjuvante Chemo- oder Radiochemotherapie erhalten. Von den Ösophaguskarzinompatienten, bei denen neoadjuvant keine Therapie stattgefunden hat, waren $17 \%$ in einem lokal fortgeschrittenen UICC-Stadium. Diese Patientengruppe würde davon profitieren, vortherapiert zu werden (Shapiro et al. 2015). Auch für Patienten mit einem Magenkarzinom wird ein Überlebensvorteil durch die Durchführung einer neoadjuvanten Therapie zusätzlich zur Operation beschrieben (Cunningham et al. 2006, Özer et al. 2017). Gut die Hälfte der Magenkarzinompatienten des untersuchten Kollektivs hat präoperativ eine die Operation ergänzende neoadjuvante Therapie erhalten. Bei den Patienten mit einem Magenkarzinom, bei denen keine neoadjuvante Therapie durchgeführt wurde, lag bei einem Anteil von 21\% ein lokal fortgeschrittenes UICC-Stadium vor. Diese Patienten würden ebenfalls von einer zusätzlich zur Operation durchgeführten neoadjuvanten Therapie profitieren (Mönig et al. 2009). Auch hier ergibt sich die Möglichkeit für weiterführende Untersuchungen. Die Auswertung der Daten in Bezug auf eine Fragestellung das Langzeitüberlebens dieses Kollektivs betreffend, war nicht Teil dieser Arbeit. Diese Frage könnte aber Anreiz für weitere Forschung sein.

Die in der UMG geltende hausinterne Vereinbarung des Tumorboards zur Wahl des Operationsverfahren sieht bei Patienten mit einem Ösophaguskarzinom oder der Diagnose eines AEG-Typ-I die Durchführung einer Ösophagusresektion vor, für Patienten mit einem Magenkarzinom oder einem AEG-Typ-II/-III wird als Operationsverfahren die Gastrektomie bzw. die erweiterte Gastrektomie vorgesehen. Eine japanische Studie zeigt, dass Tumoren, deren distales Ende mehr als $50 \mathrm{~mm}$ vom gastroösophagealen Übergang 
entfernt liegt, mittels erweiterter Gastrektomie behandelt werden sollten, um eine abdominale Lymphadenektomie durchzuführen (Mine et al. 2015). Bei der Mehrheit der Patienten ist es zur Anwendung von einem dieser drei Verfahren gekommen, was auf eine große Compliance entsprechend den Vereinbarungen des Tumorboards schließen lässt. Zu Abweichungen von dieser Strategie kann es kommen, wenn z. B. bei AEG-Typ-I-Tumoren eine ausgedehnte Lymphknotenlast beschrieben wird, die nicht vollständig im Strahlenfeld liegt. Warum es in Einzelfällen zu Abweichungen gekommen ist, lässt sich aber auch in diesem Fall an den vorliegenden Daten in der Retrospektive nicht sicher festlegen. Dabei wäre es durchaus von großem Interesse, ob die zugrunde gelegten Überlegungen des Therapieentscheids maßgeblichen Einfluss auf den Verlauf der Erkrankung hatten. Oppedijk et al. zeigten 2014, dass die Rezidive nur in einem geringen Prozentsatz im Bestrahlungsfeld auftraten. Sollten also Patienten mit ausgedehnter Lymphadenopathie wie oben erwähnt - mit einer Chemotherapie vorbehandelt werden, müsste - sofern die Therapie überhaupt erfolgreich ist - die Rate an Rezidiven hierdurch gesenkt werden können.

Die aktuelle Situation der Therapie von AEG-Tumoren stellt aber grundsätzlich die Problematik in der Behandlungsstrategie dar. Folgt man den Studien, so ist gegenwärtig nicht offensichtlich oder gar durch Leitlinien belegt, wie die Tumoren überhaupt standardisiert zu behandeln sind. Somit wird klar, dass z. B. AEG-Typ-I-Tumoren durchaus auch bei fehlendem Hinweis für eine ausgedehnte Lymphadenopathie perioperativ mit Chemotherapie behandelt wurden. Dies ist zwar zumeist in auswärtigen Krankenhäusern erfolgt, als falsch kann diese Therapie aber nicht gewertet werden. Entsprechend erfolgt aktuell die Therapie der Patienten mit AEG-Tumoren, wenn möglich, im Rahmen der ESOPEC-Studie. Hierbei werden die Patienten in die Arme einer perioperativen Chemotherapie bzw. einer neoadjuvanten Radiochemotherapie randomisiert (ESOPEC-Studie 2017).

Durch die Pathologie wird anhand von WHO-Kriterien postoperativ eine histologische Bestimmung der Operationsresektate vorgenommen. Bei den beiden ICD Diagnosen C15.5 - Bösartige Neubildung des Ösophagus, unteres Drittel und C16.0 - bösartige Neubildung des Magens, Kardia (ICD-10-GM 2016) liegt eigentlich eine Überschneidung vor. Befindet sich eine Neoplasie in diesem Bereich und handelt es sich bei dieser um ein Adenokarzinom, so ist zur genaueren Bestimmung eine Einteilung anhand der SiewertKlassifikation möglich. Hausintern werden die Karzinome, die entsprechend dieser Klassifikation als AEG-Typ-I-Tumor eingestuft werden wie oben beschrieben, wie ein Ösophaguskarzinom behandelt und Neoplasien vom AEG-Typ-II und -III wie ein Magenkarzinom. Kommt es nun beispielweise bei einem Patienten mit einem AEG-Typ-ITumor zu einer Ösophagusresektion, so ist es möglich, dass das Resektat durch die Pathologie als C.16.0 klassifiziert wird, obwohl klinisch und präoperativ die Diagnose C15.5 gestellt wurde. Anhand des Resektats ist es dem Pathologen nicht möglich, hier zu differenzieren. Die erhobene ICD-Diagnose kann nicht klären, wie die Therapie in 
Abhängigkeit der Lage erfolgt ist, da ein Rückschluss des pathologischen Befundes auf die tatsächliche Lokalisation nicht möglich ist. Zur Beurteilung der Güte der präoperativen Lokalisationsdiagnostik ist somit der aktuelle pathologische Standardbefund nicht ausreichend. Für die behandelnde Abteilung ist es von Bedeutung zu wissen, ob die von Ihnen getroffene klinische Einteilung den AEG-Typ betreffend richtig gestellt wurde. Damit dies möglich ist, müsste postoperativ durch die Pathologie Stellung zur AEGKlassifikation genommen werden um hier eine Verifizierung zu ermöglichen. Diese Verifizierung konnte retrospektiv nicht durchgeführt werden, da die benötigten Daten zur AEG-Klassifizierung sowohl von klinischer Seite als auch von Seiten der Pathologie fehlen. Im vorliegenden Kollektiv lag bei 59\% der Patienten ein Magenkarzinom vor. In dieser Gruppe handelte es sich bei 93\% der Tumoren um Adenokarzinome. Eine spezifischere Unterteilung dieser Adenokarzinome in Karzinome vom intestinalen, vom diffusen, vom tubulär oder vom muzinösen Typ ist möglich, erfolgte jedoch nur in $60 \%$ der Fälle. Bei 40\% der Patienten mit einem Adenokarzinom des Magens wurde dieses demnach nicht weiter spezifiziert. Die verschiedenen Typen des Adenokarzinoms unterscheiden sich hinsichtlich ihrer Prognose nicht (Sendler et al. 1997). Um eine Einheitlichkeit herzustellen, wäre es trotzdem künftig wünschenswert, entweder eine genauere Beschreibung aller histologischen Präparate zu erhalten oder auf eine detaillierte Befundung gänzlich zu verzichten.

Im Rahmen der Auswertung der postoperativen Befunde der Pathologie wurde die Zahl der entfernten Lymphknoten ermittelt sowie die Anzahl der befallenen Lymphknoten. Die entsprechenden Leitlinien für das Ösophagus- bzw. Magenkarzinom geben keine Mindestanzahl von zu resezierenden Lymphknoten vor. Sowohl für Ösophaguskarzinome als auch Magenkarzinome bzw. AEG-Tumoren gibt es Daten, dass die Zahl der entfernten wie auch die Ratio von befallenen zu unbefallenen Lymphknoten eine Rolle spielt. So zeigten z. B. Peyre et al., dass das Entfernen von 23 oder mehr regionalen Lymphknoten sich positiv auf die Überlebenswahrscheinlichkeit von Patienten mit einem Ösophaguskarzinom auswirkt (Peyre et al. 2008b). Im Kollektiv der Ösophaguskarzinompatienten war dies bei 19\% der Patienten der Fall. Für das Ösophaguskarzinom wiederum zeigte die Studie, dass bei drei oder mehr befallenen Lymphknoten die Wahrscheinlichkeit einer systemischen Erkrankung auf über 50\% steigt. Sind acht oder mehr Lymphknoten befallen, kommt es bei gut $90 \%$ der Patienten zu einer systemischen Erkrankung (Peyre et al. 2008a). Im entsprechenden Kollektiv lag bei 16\% der Patienten die Zahl der befallenen Lymphknoten zwischen drei und sieben und bei 2\% der Patienten sind acht oder mehr befallene Lymphknoten entfernt worden. Grundsätzlich sind somit Einordnungen in die Risikokonstellation anhand von Lymphknoten zu treffen. Es fällt jedoch auf, dass keinerlei Lokalisation der Lymphknotenmetastasen an dem jeweiligen Präparat erfolgt. Dies ist insbesondre für die Ösophagusresektionen von Bedeutung. Diese beinhalten die Lymphknoten aus dem mittleren und unteren Mediastinum sowie entlang der kleinen Kurvatur bis zum Umschlagspunkt der 
Blutversorgung. Darüber hinaus sind die Lokalisationen entlang der Arteria gastrica sinistra und die verbleibenden Lymphknotenstationen bei D2 Lymphadenektomie zu nennen. Für Gastrektomien ist eine Einteilung der Lymphknotenstationen in der histopathologischen Aufarbeitung bisher ebenfalls nicht etabliert. In Anbetracht der Unsicherheit über die Behandlung aber v. a. auch über die Diagnostik der AEG-Tumoren wäre eine Beurteilung der genannten Lymphknotenstationen von großer Bedeutung. Dies könnte für die Beurteilung der Lokalisation bzw. die zu wählende Therapie zukünftig eine relevante Rolle spielen. Auch der Wahl über die Art des Zugangsweges zur Resektion des Tumors und der betreffenden Lymphknoten wird zukünftig eine besondere Rolle zukommen. So konnte beispielweise gezeigt werden, dass bei AEG-Typ-II-Tumoren die Resektion der mittleren und tiefen Lymphknoten durch die abdominothorakale Ösophagusresektion einen Vorteil gegenüber anderen Resektionswegen bietet (Duan et al. 2017).

Im Kollektiv der Patienten, bei denen mindestens eine Ösophagogastroskopie an der Universitätsmedizin Göttingen durchgeführt wurde, konnte in 15,8\% der Fälle anhand der histomorphologischen Befundung nach der Operation ein Adenokarzinom des Ösophagus bestimmt werden. Lediglich bei knapp der Hälfte dieser Patienten lag prä- bzw. postoperativ die Beschreibung einer Barrett-Mukosa vor. Die Tatsache, dass etwa 90\% der Adenokarzinome auf der Grundlage einer Veränderung im Sinne einer Barrett-Mukosa entstehen (Bollschweiler und Hölscher 2000), zeigt deutlich, dass die Befunde diesbezüglich zukünftig eine bessere Erfassung bieten sollten.

Im Rahmen der Datenerhebung erfolgte eine Erfassung über die Durchführung adjuvanter Chemo- und Strahlentherapien. Für Patienten mit einem Ösophaguskarzinom fehlt das Vorliegen einer klaren Indikation für die Durchführung einer solchen postoperativen Therapieergänzung (Stein et al. 2004). Im entsprechenden Patientenkollektiv kam es dennoch in 14\% der Fälle zu einer adjuvanten Behandlung. Bei 1/3 der Patienten mit einem Magenkarzinom wurde nach der Operation eine adjuvante Behandlung durchgeführt. Es wird somit deutlich, dass die Idee der perioperativen Chemotherapie im klinischen Alltag häufig nicht durchgeführt wird. Dabei ist jedoch festzuhalten, dass es bei Patienten mit einem Magenkarzinom keine allgemeine Empfehlung für die Durchführung einer adjuvanten Therapie gibt (Janunger et al. 2002). Dass es einen Überlebensvorteil für Patienten der westlichen Welt gibt, die nach einer Operation mit R0-Resektion auch eine postoperative chemotherapeutische Behandlung erhalten haben konnte in Studien nicht gezeigt werden (Wilke et al. 2008).

\section{Mögliche Fehlerquellen}

Bei dieser Dissertation handelt es sich um eine retrospektive Arbeit, die sich mit der Analyse von Daten eines Patientenkollektivs aus einem Zeitraum von fünf Jahren befasst. Aus dieser Art der Studie ergeben sich mögliche Fehlerquellen, welche sich auf die Ergebnisse auswirken können. So ist die Dokumentation der Daten bei retrospektiven Arbeiten abgeschlossen, und es gibt keine Möglichkeit mehr, auf die Qualität dieser Daten 
Einfluss zu nehmen. Daraus ergibt sich das Problem, dass es sich vielfach um unvollständige Aufzeichnungen handelt und nicht bei allen Patienten des Gesamtkollektivs alle für die Auswertung benötigten Daten erfasst werden können. Des Weiteren handelt es sich bei vielen der erfassten Daten um Angaben, welche von der subjektiven Beschreibung einzelner Personen abhängig sind, da keine standardisierte Datenerhebung stattgefunden hat. In dieser Arbeit hat sich gezeigt, dass diese Tatsache vor allem bei der Auswertung der Anamnesebögen sowie der Ösophagogastroskopiebefunde problematisch war. Um die Aussagen der vorliegenden Befunde trotzdem möglichst einheitlich zu erfassen, wurden diverse Kategorien erstellt, in welche die Daten eingetragen wurden. Dies hat es ermöglicht, sie miteinander zu vergleichen und in Beziehung zueinander zu stellen. Damit die erhobenen Ergebnisse ein repräsentatives Bild wiedergeben, wurde ein Zeitraum von fünf Jahren gewählt. Es ist jedoch festzuhalten, dass sich in einem Zeitraum von fünf Jahren sowohl im diagnostischen als auch im therapeutischen Management Änderungen ergeben und dass sich das prä- und postoperative Vorgehen wandelt. Daher müssen Ergebnisse stets unter Einbeziehung dieses Wissens interpretiert werden. Auch das für diese Arbeit untersuchte Patientenkollektiv birgt Fehlerquellen. So ist bei relativ kleinen Gruppen zu beachten, dass eine gewisse Anfälligkeit für „Ausreißer“ gegeben ist. Diese Tatsache kann die Auswertung für Kollektive mit kleinen Fallzahlen verfälschen. Des Weiteren kommen die für diese Studie eingeschlossenen Patienten zumeist aus der Stadt Göttingen oder dem näheren Göttinger Umland. Somit sind regionale Einflussmöglichkeiten wie Umweltfaktoren, Gewohnheiten in der Ernährung und das in der Bevölkerung verbreitete Gesundheitsbewusstsein von Bedeutung. Ob Menschen die ihnen angebotenen Vorsorgeuntersuchungen nutzen oder nicht und ob sie sich bei auftretenden Beschwerden an einen Arzt wenden, hängt unter anderem von ihrem Bildungsstand ab (Bundeszentrale für politische Bildung 2016). Dieses Verhalten steht allerdings auch im Zusammenhang mit der Mentalität einer jeweiligen Region. Ebenso ist die medizinische Versorgungsstruktur von Bedeutung. So ist im Verhältnis zur Chemotherapie die Möglichkeit einer Bestrahlung durch das reduzierte Angebot geringer. Dies spiegelt sich auch in der Therapie wider. Somit können die erhobenen Ergebnisse nur bedingt mit denen aus anderen Regionen oder der Gesamtbevölkerung von Deutschland verglichen werden. Nichtsdestoweniger ist, unter Berücksichtigung dieses Wissens, eine Gegenüberstellung dieser Daten interessant und kann helfen, neue Erkenntnisse zu gewinnen. 


\section{$5 \quad$ Zusammenfassung}

Ösophagus- und Magenkarzinome finden sich sowohl bei weiblichen als auch bei männlichen Patienten unter den zwanzig häufigsten Krebstodesursachen in Deutschland und machen 4,4\% der bösartigen Neubildungen hierzulande aus. Da Frühsymptome selten auftreten, kommt es in vielen Fällen erst in einem fortgeschrittenen Tumorstadium zur Diagnosesicherung. Um dennoch eine für den Einzelnen optimale Therapieplanung vornehmen zu können, kommt den zur Verfügung stehenden Stagingmaßnahmen ein hohes Maß an Bedeutung zu.

Diese Erkenntnis veranlasste zu dem Vorhaben, die Patientengruppe, die mit diesen beiden Krankheitsbildern in der Universitätsmedizin Göttingen behandelt wurde, näher zu untersuchen. Für diese retrospektive Arbeit wurden die Akten von insgesamt 246 Patienten ausgewertet, die im Zeitraum von 2007 - 2012 in der UMG behandelt wurden. Dafür wurden zum einen Daten über allgemeine Faktoren wie Geschlecht, Alter, BMI und Risikofaktoren erhoben und zum anderen Daten über die im Rahmen des Stagings durchgeführten Untersuchungen und deren Befunde. Des Weiteren wurden Daten über gegebenenfalls neoadjuvant und adjuvant durchgeführte Therapien, Operationen und die Befunde der Histologie erfasst.

Bei $42 \%$ der Patienten des untersuchten Kollektivs lag die Diagnose eines Ösophaguskarzinoms vor und bei 58\% der Patienten die Diagnose eines Magenkarzinoms. Im Vergleich zu den von der GEKID veröffentlichten Daten ist das Ösophaguskarzimom in dem bearbeiteten Kollektiv etwas häufiger aufgetreten. Männer waren insgesamt etwa dreimal so oft betroffen wie Frauen. Bei der Altersanalyse zeigte sich, dass die Patienten etwas jünger waren als im bundesdeutschen Durchschnitt. Mit 42\% waren minimal mehr leicht übergewichtige Patienten im Kollektiv vertreten als normalgewichtige Patienten, deren Anteil bei 41\% lag. Risikofaktoren konnten, je nach Art, bei 15 bis 36\% der Patienten ausgemacht werden. Dies lässt Rückschlüsse darauf ziehen, dass bei der Anamneseerhebung oftmals nur eine unzureichende Erhebung und Dokumentation erfolgt ist. Als apparative Diagnostik wurde bei einem Großteil des Kollektivs eine Ösophagogastroskopie durchgeführt. Diese fand in diversen medizinischen Einrichtungen statt und wurde bei gut der Hälfte der Patienten wiederholt. Die Auswertung der Befunde zeigte zum Teil sehr lückenhafte Dokumentationen bezüglich der Tumorlokalisation, der Beschreibung anatomischer Gegebenheiten und der Zuordnung zur Siewert-Klassifikation. Die auswärtigen - also nicht an der UMG durchgeführten Untersuchungen - sind in vielen Fällen nicht aussagekräftig genug, um im behandelnden Zentrum eine Planung der Therapie zu ermöglichen. Bei den auswärtig durchgeführten Endoskopien ist bislang die Dokumentation wichtiger Informationen so lückenhaft, dass aktuell meist eine ReEndoskopie in der UMG notwendig ist. Somit stellt sich die Frage, wie die Validität der 
auswärtig durchgeführten Untersuchung zu verbessern ist. Diese grundsätzlich nicht durchzuführen, wäre jedoch keine Lösung, da eine entsprechende Zuweisung des Patienten in die primär behandelnde Abteilung weiterhin erfolgen sollte. Es wäre wünschenswert, mit einer Vereinheitlichung der Dokumentation den Standard der Untersuchungsergebnisse zu heben. Dadurch könnte eine Re-Endoskopie in einigen Fällen vermieden werden. Der Vergleich von erster Ösophagogastroskopie und einer möglichen Folgeuntersuchung zeigt in vielen Punkten eine höhere Informationsdichte für die Befunde der zweiten Endoskopie, es wird jedoch auch bei dieser eine unzureichende Dokumentation durchgeführt. Des Weiteren sind teils diskrepante Aussagen in den Unterlagen zu finden. Dies macht deutlich, dass eine einheitliche Befunddokumentation fehlt, eine Option wäre die Etablierung eines Systems, welches die Minimalanforderungen an eine Endoskopie abdeckt.

Zusammenfassend zeigt sich, dass das ausgewählte Patientenkollektiv im Wesentlichen mit publizierten Datensätzen vergleichbar ist. Kleinere Abweichungen sind sicher dem Strichprobenumfang oder lokalen Besonderheiten geschuldet. Insgesamt wird offensichtlich, dass die verfügbare Datengüte lückenhaft ist und für eine standardisierte Auswertung deutlich verbessert werden müsste. Es zeigt sich aber auch die bekannte Problematik in der Diagnostik von Tumoren des gastroösophagealen Übergangs, nämlich dass die Lokalisationsangabe je nach Untersucher variiert. Dabei sind Unterschiede zwischen den Endoskopeuren nicht untersucht, sondern die UMG den auswärtigen Häusern bzw. Praxen gegenübergestellt worden. Wo die Ursache zu suchen ist, kann anhand der vorgelegten Daten ebenfalls nur schwer beurteilt werden. Grund hierfür sind fehlende Angaben, die für die Beurteilung notwendig sind. Entsprechend ist für die Zukunft zu fordern, dass neben den Werten für Tumorbeginn und -ende, Bezug zur ZLinie, Vorhandensein einer Barrett-Schleimhaut, Bezug zum Magen und dem Zwerchfell, Darstellung des Tumors in Inversion und Vorhandensein einer Hernie auch jeweils eine Kategorisierung nach der Sievert-Klassifikation erfolgt. Erst so könnten die Ursachen für eventuelle Fehlklassifikationen identifiziert werden. Darüber hinaus wird jedoch auch klar, dass die Korrektheit der Klassifikation bis jetzt nicht objektiviert werden konnte. Dies liegt daran, dass die Befunde hinsichtlich einer Einteilung entsprechend AEG-Typ-I, -II oder -III nicht aus dem histopathologischen Befund herauszulesen sind. Hier müsste eine Stellungnahme zum Bezug des Tumors zum Ösophagus, dem Magen bzw. der Kardia erfolgen. Entsprechend sollte der Versuch unternommen werden, einen Bezug zwischen histopathologischem Befund und klinischer Einschätzung herzustellen. In diesen Kontext muss der operative Eingriff eingeordnet werden. Prinzipiell ist das Resektionsausmaß an die Klassifikation im Vorfeld angelegt. Da jedoch auch diese manchmal unklar ist, kann es intraoperativ $\mathrm{zu}$ Abweichungen kommen. In diesem Zusammenhang sind auch die Lymphknotenuntersuchungen zu sehen. Das Befallsmuster kann über die Korrektheit des gewählten Resektionsverfahrens weiteren Aufschluss geben. Wenngleich es zwar einzelne Lymphknotenstationen gibt, die im Abflussgebiet übersprungen werden, so sollte dennoch 
die Hauptlast der entfernten Lymphknoten im Operationsgebiet liegen. Ein Rückschluss hierüber ist jedoch derzeit nicht möglich.

All diese Angaben werden zudem bedeutsam, wenn es um die Applikation der richtigen Therapie geht. Diese ist derzeit in Evaluation, wobei eine Unterteilung auf die verschiedenen AEG-Typen auf Grund des oben Gesagten problematisch wird. 


\section{$6 \quad$ Literaturverzeichnis}

Agha-Mir-Salim P, Beck R, Bloching M, Berghaus A (2000): Endoskopische Behandlung einer iatrogenen Ösophagusperforation1. Laryngo-Rhino-Otol 79, 39-42

Bader L, Blumenstock G, Birkner B, Leiß O, Heesemann J, Riemann JF, Selbmann H-K (2002): HYGEA (Hygiene in der Gastroenterologie - Endoskop-Aufbereitung): Studie zur Qualität der Aufbereitung von flexiblen Endoskopen in Klinik und Praxis*. Z Gastroenterol 수, 157-170

Bareiß D, Stabenow R, Müller R, Eisinger B, Stegmaier C, Däubler P, Zeitz M, Scherübl H (2002): Aktuelle Epidemiologie des Ösophaguskarzinoms und des Kardiakarzinoms in Deutschland. Dtsch Med Wochenschr 127, 1367-1374

Baretton GB, Aust DE (2017): Aktuelle Biomarker beim Magenkarzinom. Pathologe $\underline{38}$, 93-97

Behrens A, Pech O, Graupe F, May A, Lorenz D, Ell C (2011): Barrett's adenocarcinoma of the esophagus: better outcomes through new methods of diagnosis and treatment. Dtsch Arzteblatt Int 108, 313-319

Blum K, Müller U: Dokumentationsaufwand im Ärztlichen Dienst der Krankenhäuser. Bestandsaufnahme und Verbesserungsvorschläge. Untersuchung im Auftrag der Deutschen Krankenhausgesellschaft. Dt. Krankenhaus-Verlag-Gesellschaft, Düsseldorf 2003

Böcker W, Höfler HK, Lax S, Poremba C, Moll R, Tannapfel A, Probst-Hensch N: Tumorerkrankungen. In: Böcker W, Heitz PU, Aguzzi A, Böcker-Denk-Moch (Hrsg.): Pathologie. 4., vollst. überarb. Auflage; Elsevier, Urban \& Fischer, München 2008, 167-218

Bollschweiler E, Ell C (2004): Plattenepithelkarzinom und Adenokarzinom des Ösophagus: Unterschiede in Epidemiologie, Tumorbiologie, Diagnostik und Prävention. Onkologe 10, $1168-1178$

Bollschweiler EJ, Hölscher AH (2000): Deutliche Zunahme des Adenokarzinoms im Oesophagus. Dtsch Arztebl $\underline{97}$ (27) 1896-1901

Bösing N, Schumacher B, Frieling T, Ohmann C, Jungblut R, Lübke H, Böhner H, Verreet P, Röher H-D (2003): Endosonographie in der klinischen Routine beim Adenokarzinom des distalen Ösophagus und Magens: Eine ernüchternde Bilanz. Chirurg 74, 214-223

Bruns CJ (2012): Adenokarzinome des ösophagogastralen Übergangs: Diagnostische und

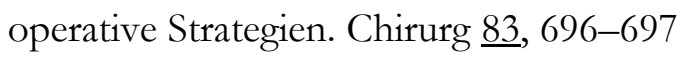


Bundeszentrale für Politische Bildung, Deutschland, Statistisches Bundesamt, Wissenschaftszentrum Berlin für Sozialforschung (Hrsg.): Datenreport 2016. Ein Sozialbericht für die Bundesrepublik Deutschland. Bundeszentrale für politische Bildung, Bonn 2016

Cunningham D, Allum WH, Stenning SP, Thompson JN, Van de Velde CJH, Nicolson M, Scarffe JH, Lofts FJ, Falk SJ, Iveson TJ, et al. (2006): Perioperative chemotherapy versus surgery alone for resectable gastroesophageal cancer. N Engl J Med $\underline{355}, 11-20$

Devesa SS, Blot WJ, Fraumeni JF (1998): Changing patterns in the incidence of esophageal

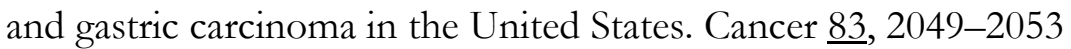

Dittler H-J (2002): Chirurgische Endoskopie für Staging und Verfahrenswahl: Chirurg $\underline{73}$, 2-8

Duan X-F, Yue J, Tang P, Shang X-B, Jiang H-J, Yu Z-T (2017): Lymph node dissection for Siewert II esophagogastric junction adenocarcinoma: A retrospective study of 3 surgical procedures. Medicine (Baltimore) $\underline{96}$, e6120

Eisen GM, Baron TH, Dominitz JA, Faigel DO, Goldstein JL, Johanson JF, Mallery JS, Raddawi HM, Vargo JJ, Waring JP, et al. (2002): Complications of upper GI endoscopy. Gastrointest Endosc 55, 784-793

Elmar T, Peuker C, Filler TJ, Pera F: Brustkorb, Thorax und Brustraum, Cavitas thoracis mit Zwerchfell, Diaphragma. In: Waldeyer AJ, Fanghänel-Pera-Anderhuber-Nitsch (Hrsg.): Anatomie des Menschen. 17., völlig überarb. Aufl; de Gruyter, Berlin 2003

Englisch-Fritz C, Hünerbein M, Porschen R (2008): Diagnostik beim Magenkarzinom. Onkologe 14, 332-338

Faller G, Berndt R, Borchard F, Ell C, Fuchs K-H, Geddert H, Gossner L, Günther T, Kirchner T, Koch HK, et al. (2003): Histopathologische Diagnostik der BarrettSchleimhaut und ihrer Neoplasien. Pathologe 24, 9-14

Fischbach, W (1997): Magenkarzinom und MALT-Lymphom: Prophylaxe durch Helicobacter-pylori-Eradikation? Dtsch Med Wochenschr 122, 983-987

Frieling T: Motilitätsstörungen von Pharynx und Ösophagus. In: Adler G, Beglinger C, Manns MP, Müller-Lissner S, Schmiegel W (Hrsg.): Klinische Gastroenterologie und Stoffwechsel. Springer, Berlin Heidelberg 2000, 61-70

Fritz P: Ösophaguskarzinom. In: Wannenmacher M, Debus J, Wenz F (Hrsg.): Strahlentherapie. Springer, Berlin Heidelberg 2006, 507-522 
Halm U, Witzigmann H: Ösophagustumoren. In: Caspary WF, Mössner J, Stein J (Hrsg.): Therapie gastroenterologischer Krankheiten. Springer, Berlin Heidelberg 2005, 24-30

Hamilton SR, Aaltonen LA, World Health Organization, International Agency for Research on Cancer (Hrsg.): Pathology and genetics of tumours of the digestive system. (World Health Organization classification of tumours). IARC Press, Lyon : Oxford 2000

Hölscher A, Fetzner U, Bollschweiler E, Mönig S (2010): Adenokarzinom des ösophagogastralen Übergangs. Allg Visz Up2date $\underline{4}$, 35-53

ICD-10-GM 2016 Systematisches Verzeichnis: Internationale statistische Klassifikation der Krankheiten und verwandter Gesundheitsprobleme. 10. Revision - German Modification. Hrsg.: Deutsches Institut für Medizinische Dokumentation und Information (DIMDI), bearb. von Bernd Graubner. Deutscher Ärzte-Verlag, Köln 2016Janunger K-G, Hafström L, Glimelius B (2002): Chemotherapy in gastric cancer: a review and updated meta-analysis. Eur J Surg Acta Chirurg 168, 597-608

Jochum W: Magen und Duodenum. In: Böcker W, Heitz PU, Aguzzi A, Böcker-DenkMoch (Hrsg.): Pathologie. 4., vollst. überarb. Auflage; Elsevier, Urban \& Fischer, München 2008, 697-715

Jochum W, Barreton G: Ösophagus. In: Böcker W, Denk H, Heitz PU, Höfler G, Kreipe H, Moch H (Hrsg.): Pathologie. 5., vollst. überarb. Auflage; Elsevier, Urban \& Fischer, München 2012, 545-554

Menges M (2004): Ösophaguskarzinom - Diagnostik. PRAXIS $\underline{93}$, 2047-2050

Meyer H-J, Öfner D: Magenkarzinom. In: Gnant M, Schlag PM (Hrsg.): Chirurgische Onkologie. Springer Vienna, Wien 2008, 173-184

Mine S, Kurokawa Y, Takeuchi H, Kishi K, Ito Y, Ohi M, Matsuda T, Hamakawa T, Hasegawa S, Yoshikawa T, et al. (2015): Distribution of involved abdominal lymph nodes is correlated with the distance from the esophagogastric junction to the distal end of the tumor in Siewert type II tumors. Eur J Surg Oncol EJSO 411, 1348-1353

Nigg C, Kolyvanos Naumann U, Käser L, Vetter W (2006): Ösophaguskarzinom. PRAXIS $\underline{95}, 175-180$

Oppedijk V, van der Gaast A, van Lanschot JJB, van Hagen P, van Os R, van Rij CM, van der Sangen MJ, Beukema JC, Rütten H, Spruit PH, et al. (2014): Patterns of Recurrence After Surgery Alone Versus Preoperative Chemoradiotherapy and Surgery in the CROSS Trials. J Clin Oncol 2, 385-391

Ott R, Lersch C, Werner M, Classen M (2000): Barrett-Ösophagus - Diagnose und 
Management. Internist 41, 802-816

Peyre CG, Hagen JA, DeMeester SR, Van Lanschot JJB, Hölscher A, Law S, Ruol A, Ancona E, Griffin SM, Altorki NK, et al. (2008a): Predicting Systemic Disease in Patients With Esophageal Cancer After Esophagectomy: A Multinational Study on the Significance of the Number of Involved Lymph Nodes. Ann Surg $\underline{248}, 979-985$

Peyre CG, Hagen JA, DeMeester SR, Altorki NK, Ancona E, Griffin SM, Hölscher A, Lerut T, Law S, Rice TW, et al. (2008b): The Number of Lymph Nodes Removed Predicts Survival in Esophageal Cancer: An International Study on the Impact of Extent of Surgical Resection: Trans Meet Am Surg Assoc 126, 190-197

Pohl H, Pech O, Arash H, Stolte M, Manner H, May A, Kraywinkel K, Sonnenberg A, Ell C (2016): Length of Barrett's oesophagus and cancer risk: implications from a large sample of patients with early oesophageal adenocarcinoma. Gut $\underline{65}$, 196-201

Püschmann H, Haferkamp G, Scheppokat KD, Vinz H, Wegner M (2006): Vollständigkeit und Qualität der ärztlichen Dokumentation in Krankenakten: Untersuchung zu Krankenunterlagen aus Chirurgie, Orthopädie, Innerer Medizin und Neurologie. Dtsch Arztbl $\underline{103}$ (3) A 121-6

Riede UN, Blum HE: Magen. In: Rieder UN, Werner M, Freudenberg N (Hrsg.): Basiswissen Allgemeine und Spezielle Pathologie; Springer, Berlin Heidelberg, 2009, 344 349

Robert Koch-Institut (2010a): Verbreitung von Krebserkrankungen in Deutschland: Entwicklung der Prävalenzen zwischen 1990 und 2010. Beiträge zur Gesundheitsberichterstattung des Bundes. RKI, Berlin 2010

RKI (2010b): Krebs in Deutschland 2005/2006: Häufigkeiten und Trends. Eine gemeinsame Veröffentlichung des Robert Koch-Instituts und der Gesellschaft der Epidemiologischen Krebsregister in Deutschland e.V. (Beiträge zur Gesundheitsberichterstattung des Bundes). 7. Ausgabe; Robert Koch-Institut, Berlin 2010

RKI (2015): Krebs in Deutschland 2011/2012. 10. Ausgabe. Robert Koch-Institut (Hrsg) und die Gesellschaft der epidemiologischen Krebsregister in Deutschland e.V. (Hrsg). Berlin 2015

Rosen R (1990): Symptome des Ösophaguskarzinoms. Eur Surg 22, 24-27

Schiesser M, Schneider PM: Surgical Strategies for Adenocarcinoma of the Esophagogastric Junction. In: Schneider PM (Hrsg.): Adenocarcinoma of the Esophagogastric Junction,. Springer, Berlin Heidelberg, 2009, 93-106 
Schmid A, Kremer B: Chirurgische Prinzipien beim Magencarcinom. In: Lobenhoffer P (Hrsg.): Chirurgie upgrade 2002. Springer, Berlin Heidelberg, 2002, 24-36

Schoppmann SF (2013): Operable gastroösophageale Adenokarzinome: Multimodale Therapiekonzepte im fortgeschrittenen Stadium. Wien Klin Mag 16, 26-31

Seehofer D, Schmidt SC, Pöllinger A, Thuss-Patience P, Badakshi H (2013): Übersicht Chirurgische Therapie des Ösophaguskarzinoms. Tumordiagn Ther $\underline{34}$, 198-201

Sendler A, Nekarda H, Böttcher K, Fink U, Siewert JR (1997): Prognosefaktoren beim Magenkarzinom. Dtsch Med Wochenschr 122, 794-800

Shapiro J, van Lanschot JJB, Hulshof MCCM, van Hagen P, van Berge Henegouwen MI, Wijnhoven BPL, van Laarhoven HWM, Nieuwenhuijzen GAP, Hospers GAP, Bonenkamp JJ, et al. (2015): Neoadjuvant chemoradiotherapy plus surgery versus surgery alone for oesophageal or junctional cancer (CROSS): long-term results of a randomised controlled trial. Lancet Oncol 16, 1090-1098

Siewert JR, Hölscher AH, Becker K, Gössner W (1987): Kardiacarcinom: Versuch einer therapeutisch relevanten Klassifikation. Chirurg $\underline{58}$, 25-32

Singer MV, Teyssen S: Alkohol und Alkoholfolgekrankheiten: Grundlagen - Diagnostik Therapie. Springer, Berlin Heidelberg 1999

Sjoquist KM, Burmeister BH, Smithers BM, Zalcberg JR, Simes RJ, Barbour A, Gebski V (2011): Survival after neoadjuvant chemotherapy or chemoradiotherapy for resectable oesophageal carcinoma: an updated meta-analysis. Lancet Oncol 12, 681-692

Souza RF, Spechler SJ (2005): Concepts in the Prevention of Adenocarcinoma of the Distal Esophagus and Proximal Stomach. CA Cancer J Clin 도, 334-351

Stamatakos M, Douzinas E, Stefanaki C, Safioleas P, Polyzou E, Levidou G, Safioleas M (2009): Gastrointestinal stromal tumor. World J Surg Oncol Z, 61

Stein HJ, Feith M, Fink U, Werner M, Roder JD: Adenokarzinom des ösophagogastralen Überganges (AEG). In: Roder JD, Stein HJ, Fink U (Hrsg.): Therapie gastrointestinaler Tumoren. Springer, Berlin Heidelberg 2000, 208-216

Stein HJ, von Rahden BHA, Höfler H, Siewert JR (2003): Karzinom des ösophagogastralen Übergangs und Barrett-Ösophagus: Ein nahezu klares onkologisches Modell? Chirurg $\underline{74}$, 703-708

Stein HJ, Lordick F, Siewert JR (2004): Ösophaguskarzinom: Chirurgische und multimodale Therapie. Visc Med 20, 13-20 
Stein HJ, von Rahden BHA, Höfler H, Siewert JR (2013): Karzinom des ösophagogastralen Übergangs und Barrett-Ösophagus. Chirurg 74, 703-708

Van Hagen P, Hulshof MCCM, van Lanschot JJB, Steyerberg EW, Henegouwen MI van B, Wijnhoven BPL, Richel DJ, Nieuwenhuijzen GAP, Hospers GAP, Bonenkamp JJ, et al. (2012): Preoperative Chemoradiotherapy for Esophageal or Junctional Cancer. N Engl J Med 366, 2074-2084

Van Soest EM (2005): Increasing incidence of Barrett's oesophagus in the general population. Gut $\underline{54}, 1062-1066$

Von Herbay A, Kreketer GR, Riede UN: Digestorisches System. In: Riede UN, Werner M, Schäfer HE (Hrsg.): Allgemeine und spezielle Pathologie: 5., komplett überarb. Auflage; Thieme, Stuttgart 2004, 649-708

Watanabe H, Jass JR, Sobin LH, Weltgesundheitsorganisation (Hrsg.): Histological typing of oesophageal and gastric tumours. (International histological classification of tumours 18), 2. ed; Springer, Berlin 1990

Wehrmann T, Riphaus A (2008): Sedation with propofol for interventional endoscopic procedures: A risk factor analysis. Scand J Gastroenterol $\underline{43}$, 368-374

Welsch U: Sobotta Lehrbuch Histologie: Zytologie, Histologie, mikroskopische Anatomie. 2., völlig überarb. Auflage, Elsevier, Urban \& Fischer, München 2009Wilke H, Willich N, Meyer H-J, Stahl M (2008): Neoadjuvante und perioperative Therapie des Magenkarzinoms. Onkologe 14, 370-380

Wittekind C, Asamura H, Sobin LH, Union for International Cancer Control (Hrsg.): TNM-Atlas: Ein illustrierter Leitfaden zur TNM/p'TNM-Klassifikation maligner Tumoren. 6. Auflage, Wiley-VCH Verlag, Weinheim 2015 


\section{Internetquellen:}

dkfz 2012: Deutsches Krebsforschungszentrum: Becker N, Holzmeier S, Die 20 häufigsten Krebstodesursachen in Deutschland im Jahr 2012 (aktualisierte Graphik): https://www.dkfz.de/de/krebsatlas/gesamt/organ.html (Zugriff am: 01.04.2017)

ESOPEC-Studie 2017: https://www.uniklinik-freiburg.de/esopec-studie.html (Zugriff am: 02.07.2017)

G-CCC (Göttinger Comprehensive Cancer Center) 2017a: Krebsregister: http://www.ccc.med.uni-goettingen.de/de/content/krebsregister.html (Zugriff am: 01.04.2017)

G-CCC (Göttinger Comprehensive Cancer Center) 2017b: Über uns: http://www.ccc.med.unigoettingen.de/de/content/ueberuns.html (Zugriff am: 01.04.2017)GEKID-Atlas 2016: Gesellschaft der epidemiologischen Krebsregister in Deutschland e.V. Atlas der Krebsinzidenz und mortalität in Deutschland (GEKID-Atlas). Datenlieferung: März 2016, Lübeck, 2016. Verfügbar über: http://www.gekid.de/Atlas/CurrentVersion/ Ergebnisse\%20GEKID\%20Atlas\%20Deutschland_2016.pdf (Zugriff am: 01.04.2017)

Gesundheitsberichterstattung des Bundes 2017: Body Mass Index (BMI) der erwachsenen Bevölkerung, Adjustierter Body-Mass-Index nach Alter, Geschlecht und sozialer Schicht, Telefonischer Gesundheitssurvey 2003/2004 (GSTel 04): http://www.gbe-bund.de/gbe10 /ergebnisse.prc_tab?fid $=8397 \&$ suchstring $=$ bmi\&query_id $=\&$ sprache $=D \&$ fund_typ $=\mathrm{TAB}$ $\&$ methode $=2 \& v t=1 \& v e r w a n d t e=1 \&$ page_ret $=0 \&$ seite $=\& p \_l f d \_n r=2 \& p \_n e w s=\& p \_s p r a$ chkz=D\&p_uid=gast\&p_aid=90018891\&hlp_nr=3\&p_janein=J\#tab3 (Zugriff am: 02.04.2017)

Leitlinie Magenkarzinom (2012): S3 Leitlinie Diagnostik und Therapie der Adenokarzinome des Magens und ösophagogastralen Übergangs, Langversion 02.2012, AWMFRegisternummer: 032/009OL, http://leitlinienprogramm-onkologie.de/Leitlinie.7.0.html (Zugriff am: 02.11.2016)

Leitlinie Ösophaguskrzinom (2015): S3 Leitlinie Diagnostik und Therapie der Plattenepithelkarzinome und Adenokarzinome des Ösophagus, Langversion 1.0, 2015, AWMF Registernummer: 021/023OL, http://leitlinienprogramm-onkologie.de/ Leitlinien.7.0.html, (Zugriff am: 02.11.2016)

Mönig SP, Alakus H, Metzger R, Waldschmidt D, Weihrauch M, Drebber U, Bollschweiler E, Hölscher AH (2009): MAGIC-Trial beim Magenkarzinom: klinische Ergebnisse mit Responseevaluation und Komplikationsanalyse. https://www.egms.de/static/en/ meetings/dgch2009/09dgch752.shtml (Zugriff am: 02.11.2016) 


\section{Danksagung}

Prof. Dr. Jochen Gaedcke danke ich sehr herzlich für die Bereitstellung des Themas dieser Dissertation sowie für die gute Betreuung und Zusammenarbeit.

Für die ersten gemeinsamen Überlegungen auf dem Weg zur Planung dieser Arbeit danke ich Dr. Junius Salendo und Francisca Hudemann. Ihr danke ich zudem für die gemeinsame Archivarbeit.

Mein Dank gilt zudem den Mitarbeiterinnen und Mitarbeitern des Zentralarchivs der Universitätsmedizin Göttingen für die unkomplizierte Bereitstellung der Akten.

Bei Dr. Eva Noack, Dr. Julia Proeger, Dr. Monika Weig und Dr. Till Proeger bedanke ich mich für ihre freundschaftliche Unterstützung sowie ihre inhaltlichen und methodischen Anregungen. 